\title{
Copper-catalyzed anomeric $O$-arylation of carbohydrate derivatives at room temperature
}

Tristan Verdelet, ${ }^{\mathrm{a}}$ Sara Benmahdjoub, ${ }^{\mathrm{b}}$ Belkacem Benmerad, ${ }^{\mathrm{b}}$ Mouad Alami ${ }^{\mathrm{a}}$ and Samir Messaoudi*a

a Univ Paris-Sud, CNRS, BioCIS-UMR 8076, Laboratoire de Chimie Thérapeutique, LabEx LERMIT, Faculté de Pharmacie, 5 rue J.-B. Clément, Châtenay-Malabry, F-92296, France.; Tel: + (33) 0146835683; E-mail: samir.messaoudi@u-psud.fr

${ }^{b}$ Laboratoire de Physico-Chimie des Matériaux et Catalyse, Faculté des Sciences Exactes, Université de Bejaia, 06000 Bejaia, Algeria 


\section{Table of content}

Full optimization table $\quad$ S3

NMR data of coupling products 3a-d, 3f-p and 4a-i

NMR data of synthesized starting materials $\mathbf{1 b}, \mathbf{1 d}, \mathbf{1 f}, \mathbf{1} \mathbf{h}, \mathbf{1} \mathbf{i}$ and $\mathbf{1} \mathbf{j}$

HPLC chromatograms of compounds $\mathbf{4 f}$, $\mathbf{4 h}$ and $\mathbf{4 i}$. S56

List of starting boronic acids that did not provide the desired coupling product. $\quad$ S59

Study of the stability of starting material $1 \mathrm{a}$ and both anomers of product $3 \mathrm{a}$ in $\quad \mathrm{S} 60$

$\mathrm{CD}_{2} \mathrm{Cl}_{2}$

Table of literature comparison of a selection of the glycoside products

S61 


\section{Full optimization table.}

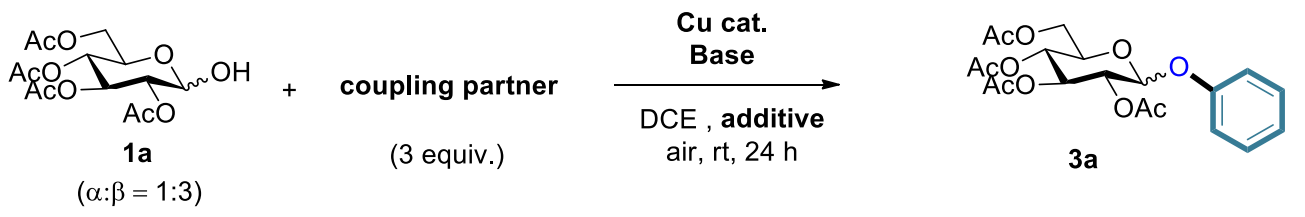

\begin{tabular}{|c|c|c|c|c|c|}
\hline Coupling partner & Catalyst & Base & Additive & Yield (\%) ${ }^{\mathrm{a}}$ & $\alpha: \beta$ ratio $^{b}$ \\
\hline $2 a$ & $\mathrm{Cu}(\mathrm{OAc})_{2}{ }^{*} \mathrm{H}_{2} \mathrm{O}$ & Pyridine & no & 32 & $1: 1.2$ \\
\hline $2 a$ & $\mathrm{Cu}(\mathrm{OAc})_{2} * \mathrm{H}_{2} \mathrm{O}$ & Pyridine & no & 39 & $1: 1.2$ \\
\hline $2 a$ & $\mathrm{Cu}(\mathrm{OAc})_{2} * \mathrm{H}_{2} \mathrm{O}$ & DMAP & no & 15 & $1: 2.8$ \\
\hline $2 a$ & $\mathrm{Cu}(\mathrm{OAc})_{2}{ }^{*} \mathrm{H}_{2} \mathrm{O}$ & 2,6-lutidine & no & 8 & $1: 3$ \\
\hline $2 a$ & $\mathrm{Cu}(\mathrm{OAc})_{2}{ }^{*} \mathrm{H}_{2} \mathrm{O}$ & Et3N & no & - & - \\
\hline $2 a$ & $\mathrm{Cu}(\mathrm{OAc})_{2} * \mathrm{H}_{2} \mathrm{O}$ & $\mathrm{K} 2 \mathrm{CO} 3$ & no & - & - \\
\hline $2 a$ & $\mathrm{Cu}(\mathrm{acac})_{2}$ & Pyridine & no & 10 & $3: 1$ \\
\hline $2 a$ & $\mathrm{Cu}(\mathrm{OTf})_{2}$ & Pyridine & no & 11 & Only $\beta$ \\
\hline $2 a$ & $\mathrm{CuO}$ & Pyridine & no & - & - \\
\hline $2 a$ & $\mathrm{Cu}(\mathrm{OAc})_{2} * \mathrm{H}_{2} \mathrm{O}$ & Pyridine & M.S. 4Å & 73 & $1: 3$ \\
\hline $2 a$ & $\mathrm{Cu}(\mathrm{OAc})_{2}{ }^{*} \mathrm{H}_{2} \mathrm{O}$ & Pyridine & M.S. $4 \AA$ & $84^{c}$ & $1: 2$ \\
\hline $2 a$ & $\mathrm{Cu}(\mathrm{OAc})_{2}$ & Pyridine & M.S. $4 \AA$ & $82^{c}$ & $1: 2$ \\
\hline B2 & $\mathrm{Cu}(\mathrm{OAc})_{2}{ }^{*} \mathrm{H}_{2} \mathrm{O}$ & Pyridine & no & - & - \\
\hline B3 & $\mathrm{Cu}(\mathrm{OAc})_{2} * \mathrm{H}_{2} \mathrm{O}$ & Pyridine & M.S. 4Å & - & - \\
\hline B4 & $\mathrm{Cu}(\mathrm{OAc})_{2} * \mathrm{H}_{2} \mathrm{O}$ & Pyridine & M.S. 4Å & - & - \\
\hline
\end{tabular}

aReaction conditions : 1a (1 equiv), coupling partner (3 equiv), [Cu] (20 mol \%), base (1 equiv), $24 \mathrm{~h}, \mathrm{rt}$, [0.5M], yield of isolated $3 \mathrm{a}$ as a mixture of both anomers. ${ }^{\mathrm{b}} \mathrm{Anomer}$ ratio was measured by ${ }^{1} \mathrm{H}$ NMR of the crude mixture and found to be the same when calculated after purification of both $\alpha: \beta$ anomers. ${ }^{c} 30 \mathrm{~mol} \%$ of $\mathrm{Cu}(\mathrm{OAc})_{2} \cdot \mathrm{H}_{2} \mathrm{O}$ was used and the reaction concentration increased into $[0.7 \mathrm{M}]$.

\section{Coupling partners ${ }^{1}$}<smiles>COc1ccccc1</smiles>

$2 a$<smiles>FC(F)(F)c1ccccc1</smiles>

B2

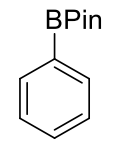

B3

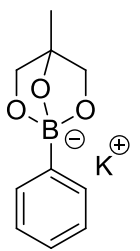

B4

\footnotetext{
1 Reagents 2a, B2 and B3 were bought from available commercial sources. Compound B4 was prepared according to the procedure described by S. Sakashita, M. Takizawa, J. Sugai, H. Ito, Y. Yamamoto, Org.Lett. 2013, 15, 4308-4311. Characterization data were in good agreement with the literature.
} 


\section{NMR data of coupling products 3a-d, 3f-p and 4a-i}

1-Phenyl-2,3,4,6-tetra-O-acetyl-a-D-glucopyranoside ن
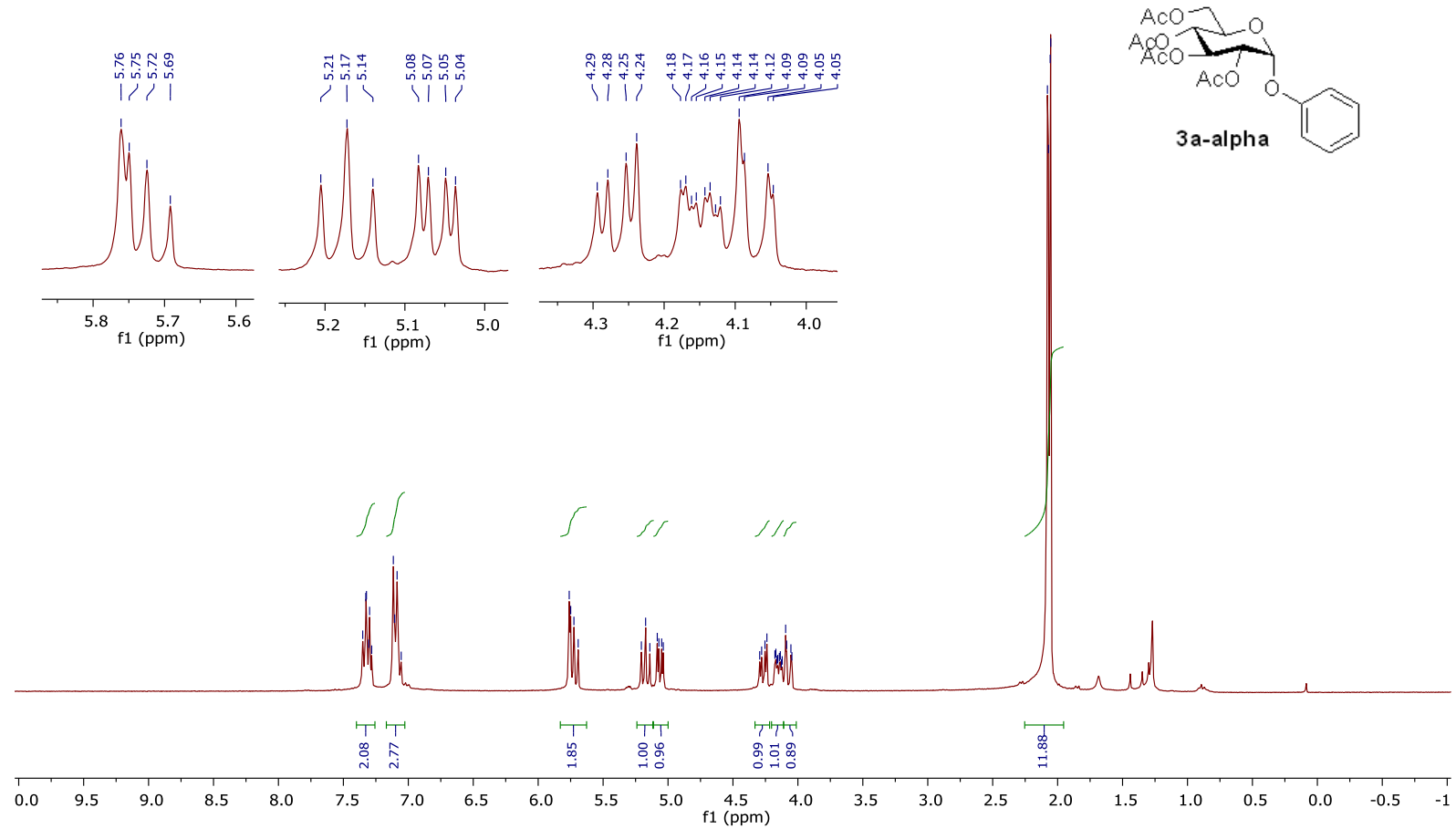

1-Phenyl-2,3,4,6-tetra-O-acetyl-a-D-glucopyranoside

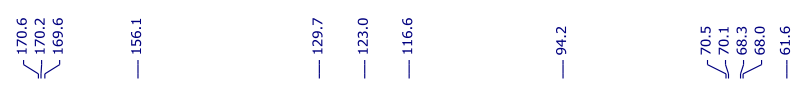

AcO?

3a-alpha

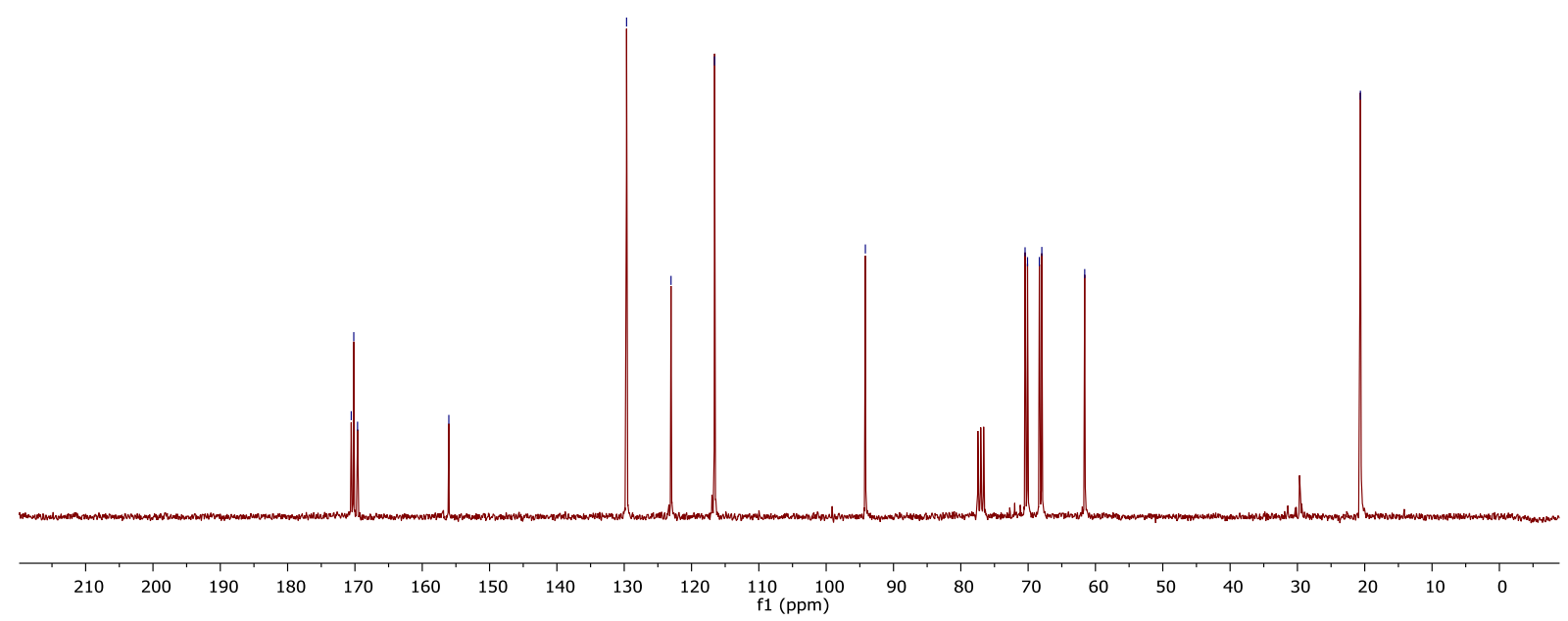




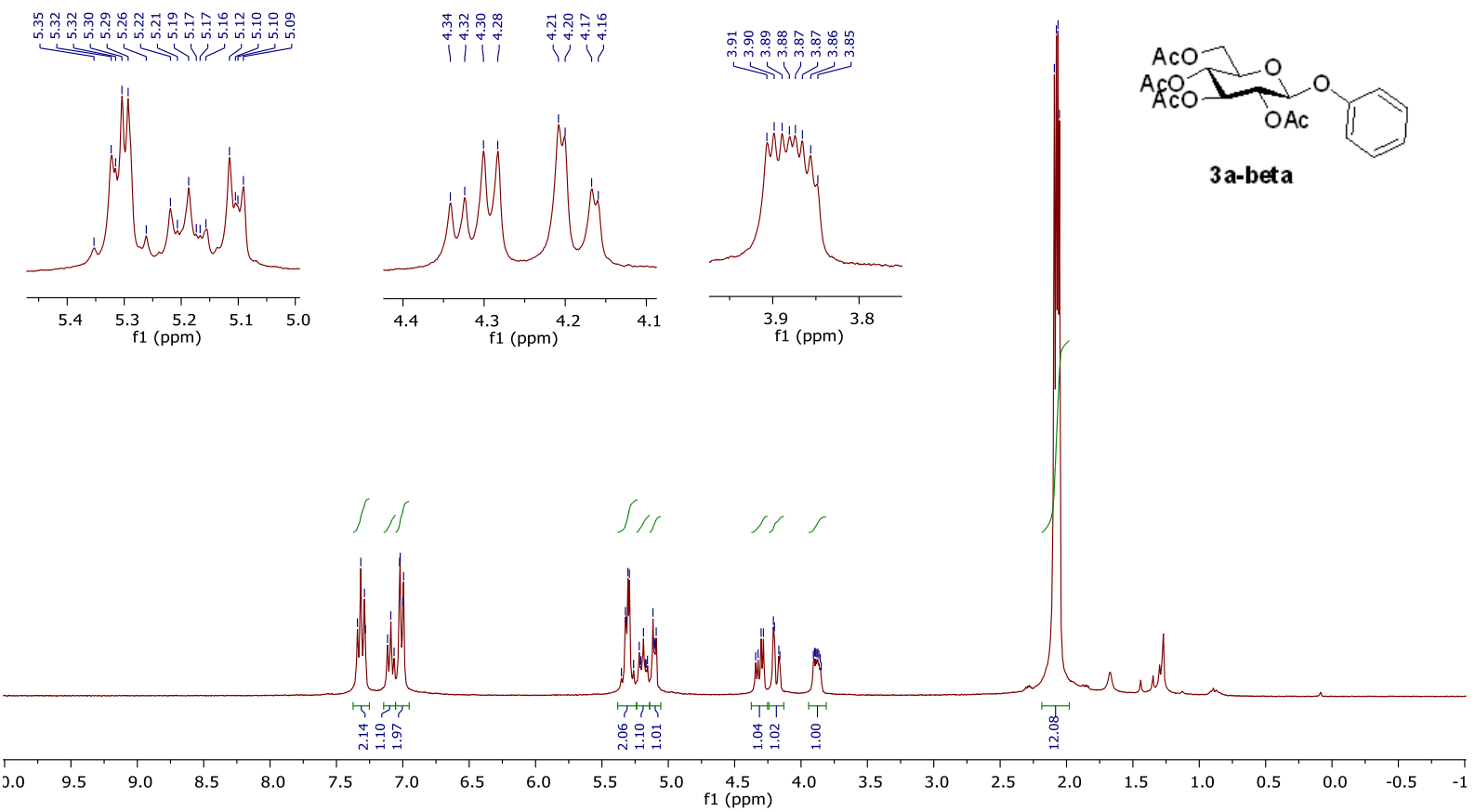

1-Phenyl-2,3,4,6-tetra-O-acetyl-b-D-glucopyranoside

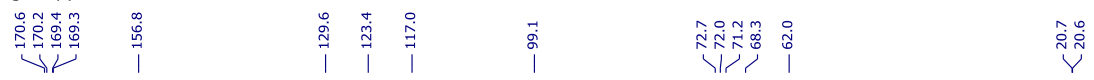

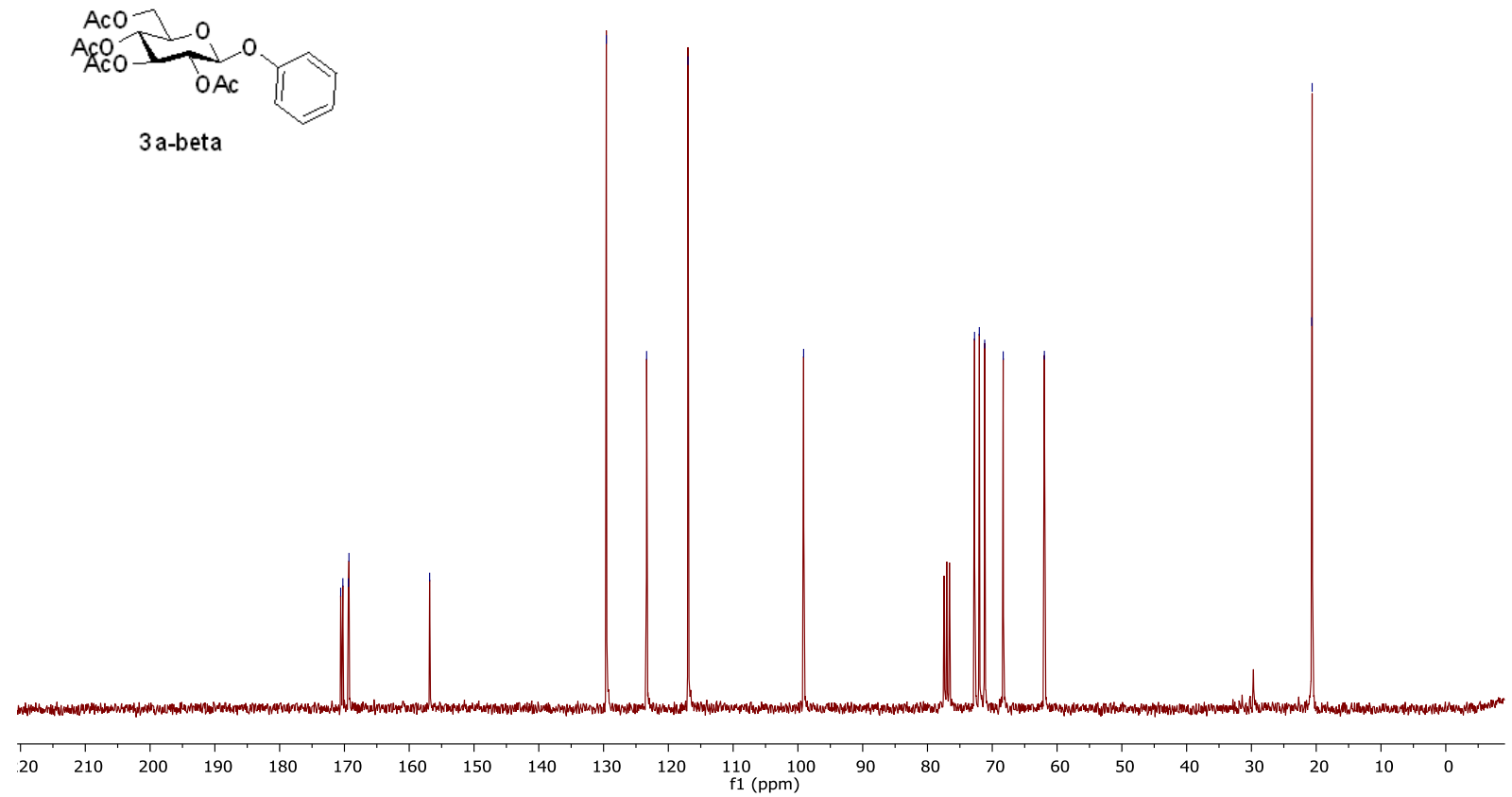




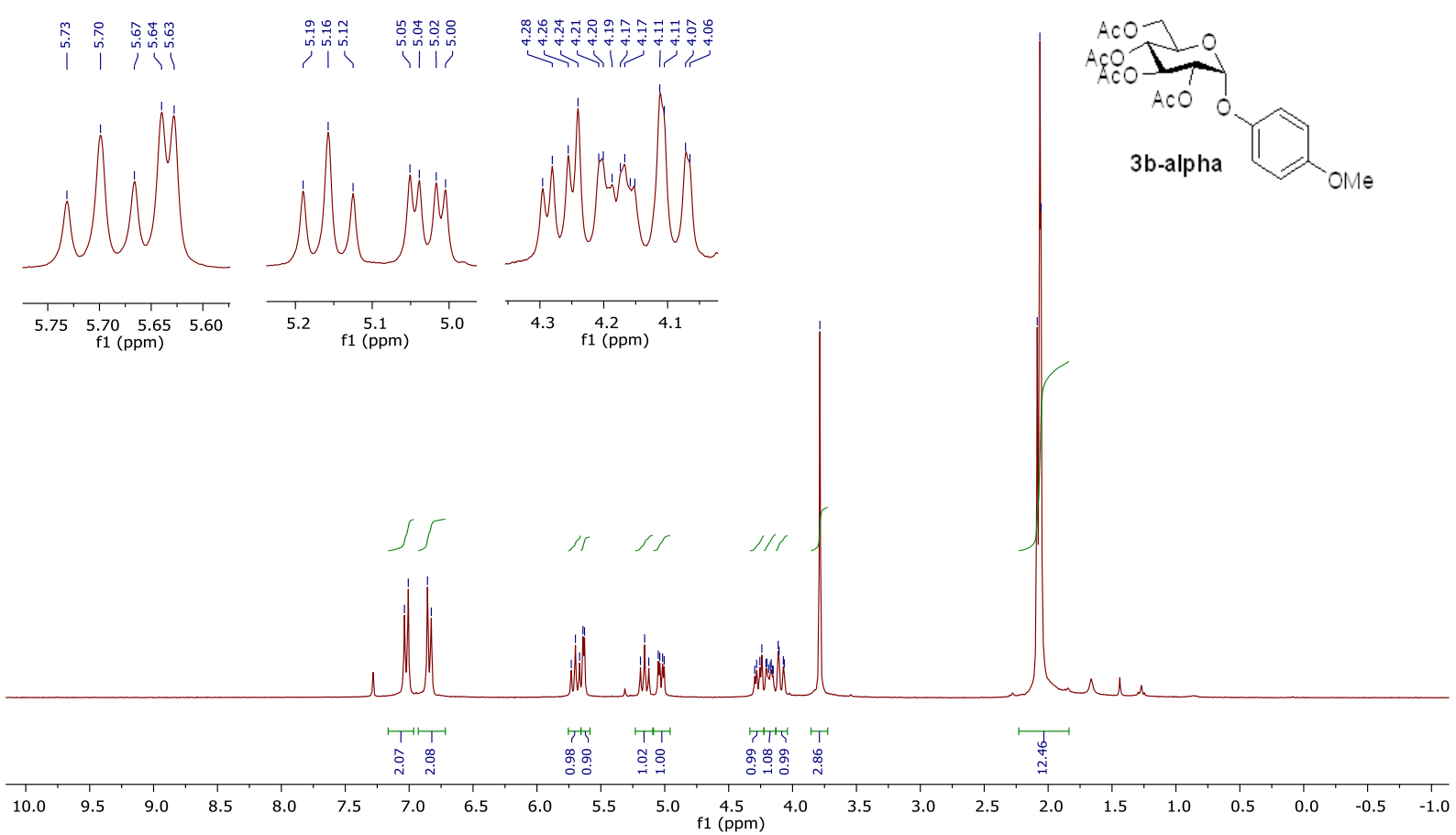

4'-Methoxy 2,3,4,6-tetra-O-acetyl-a-D-glucopyranoside

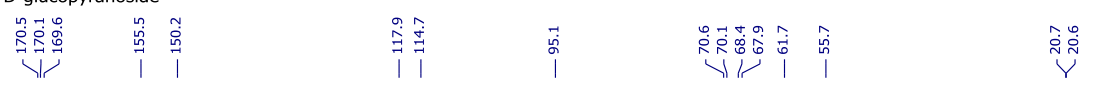
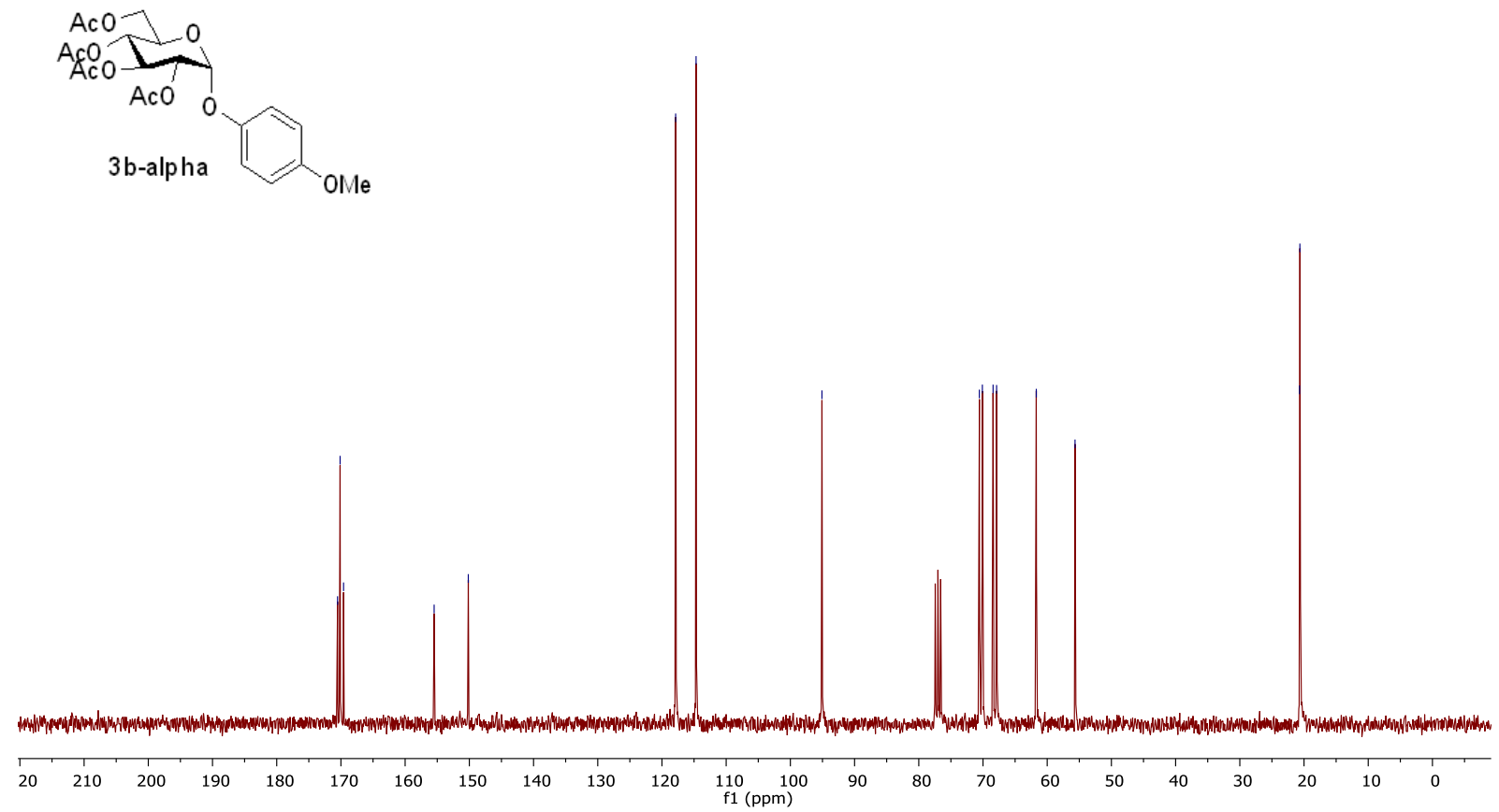
4'-Methoxy 2,3,4,6-tetra-O-acetyl-b-D-glucopyranoside

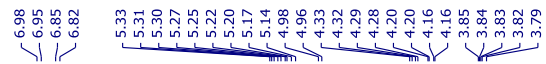

ำำำำำ
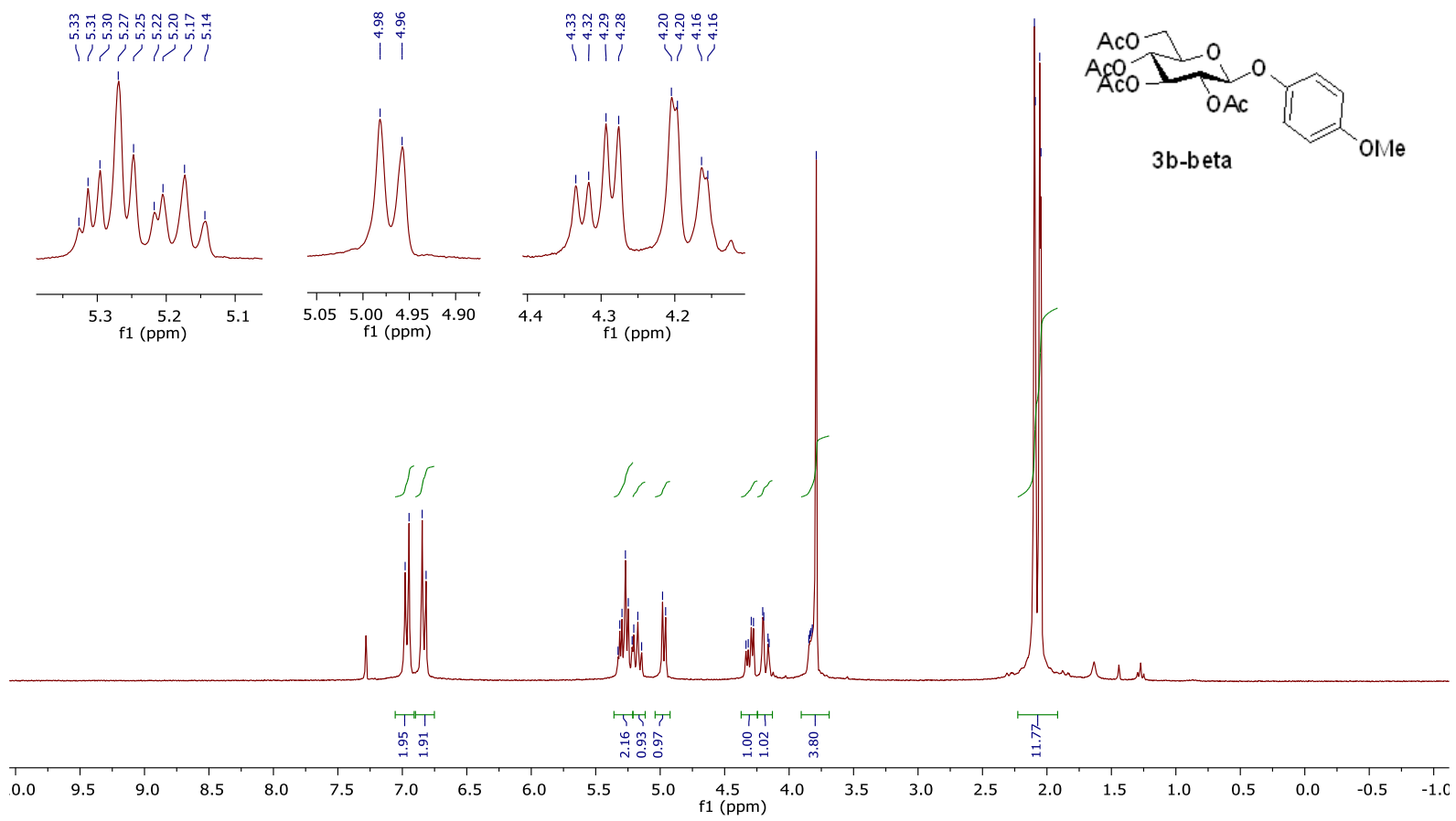

4'-Methoxy 2,3,4,6-tetra-O-acetyl-b-D-glucopyranoside

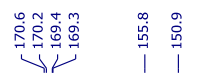

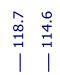

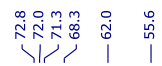

บิํํำ
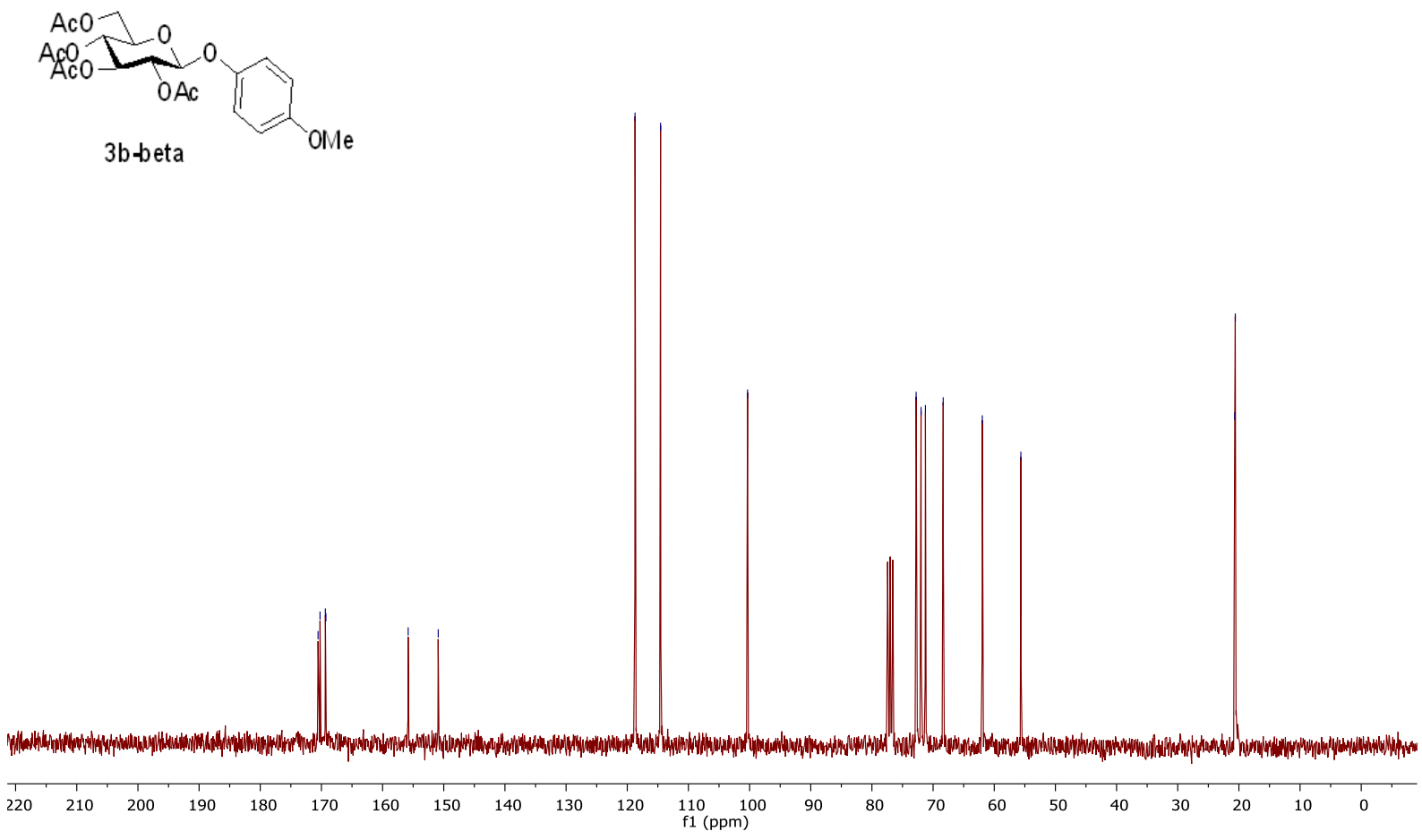

S7 


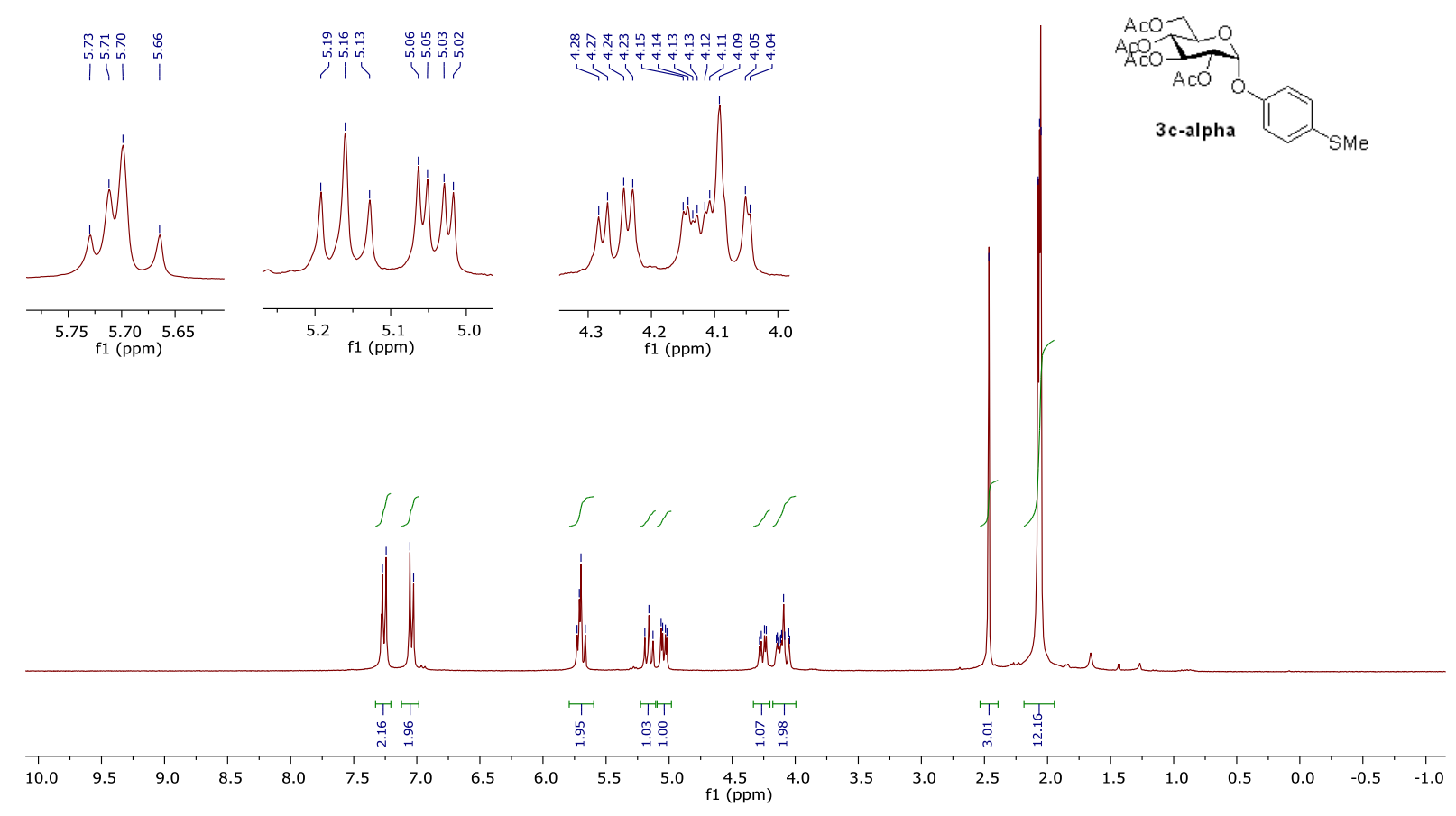

4'-Thiomethyl 2,3,4,6-tetra-O-acetyl-a-D-glucopyranoside

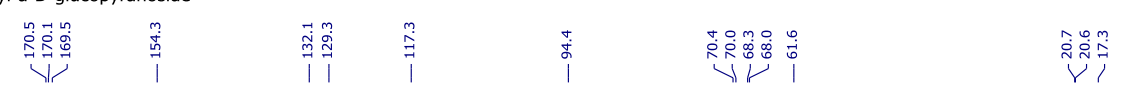
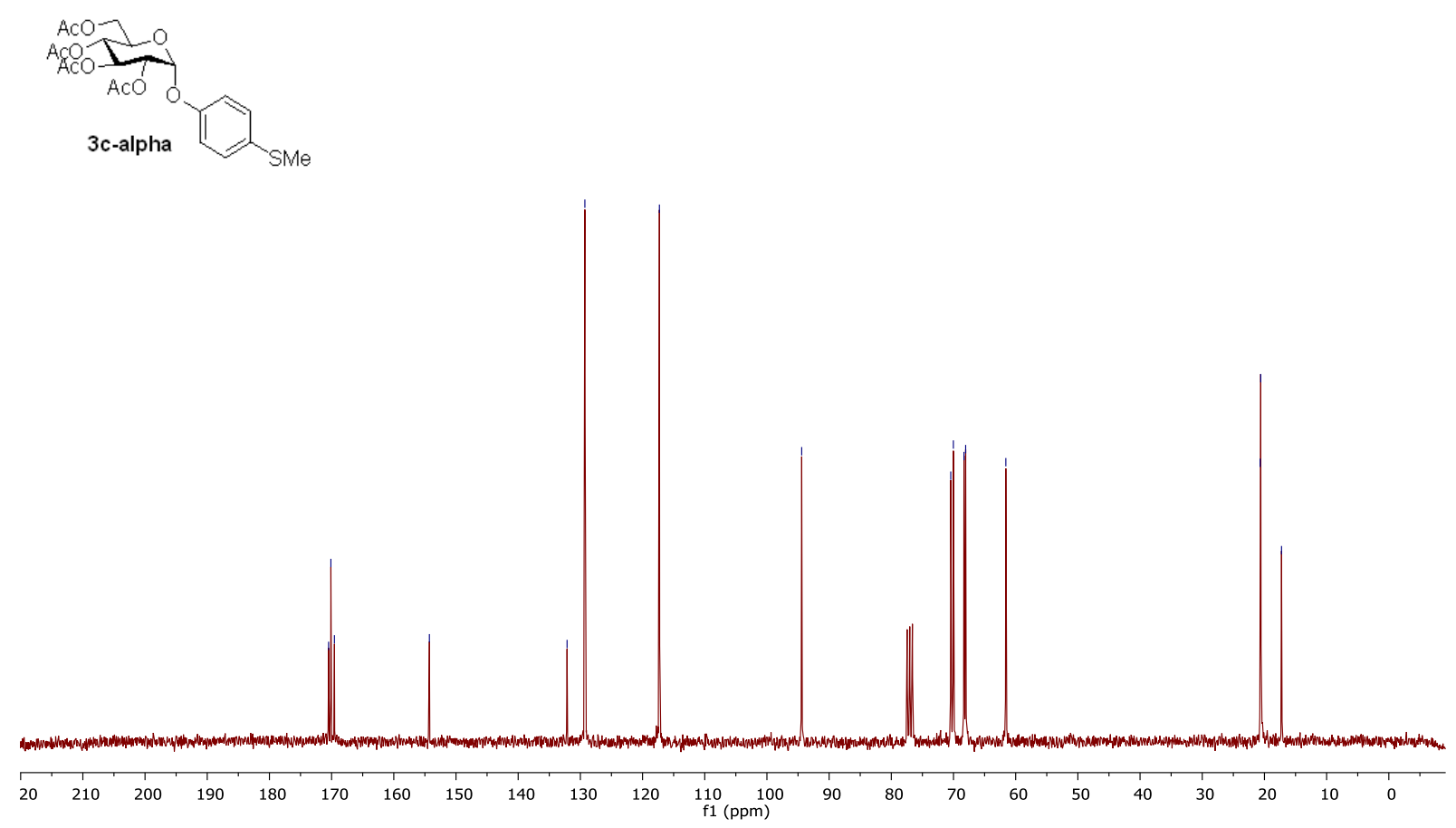


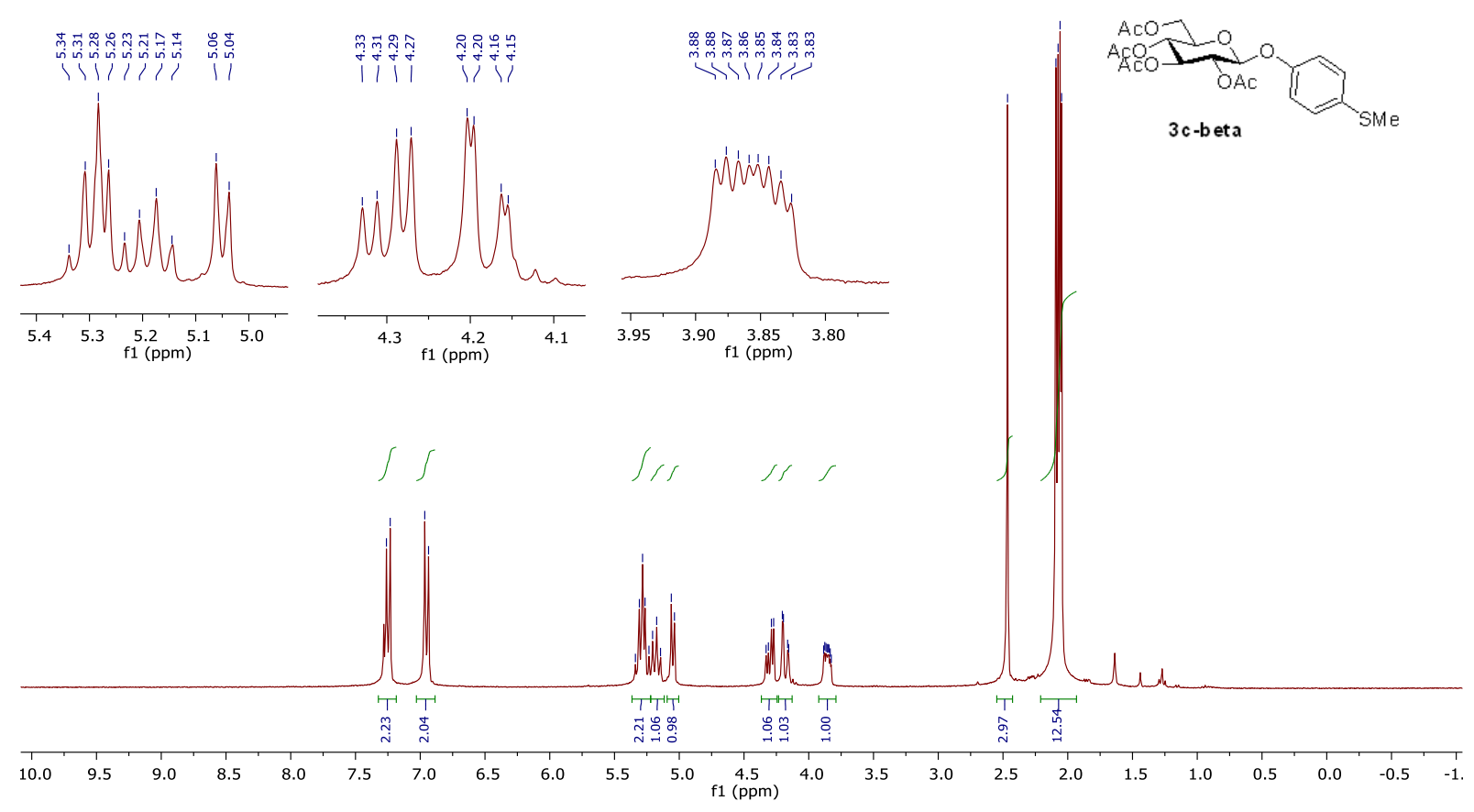

1-(4'-Thiomethyl)-phenyl-2,3,4,6-tetra-O-acetyl-b-D-glucopyranoside

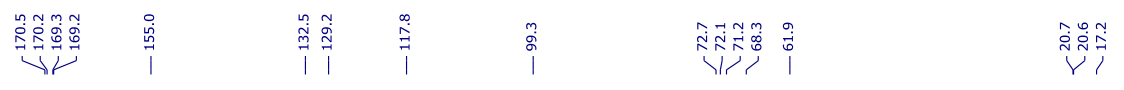

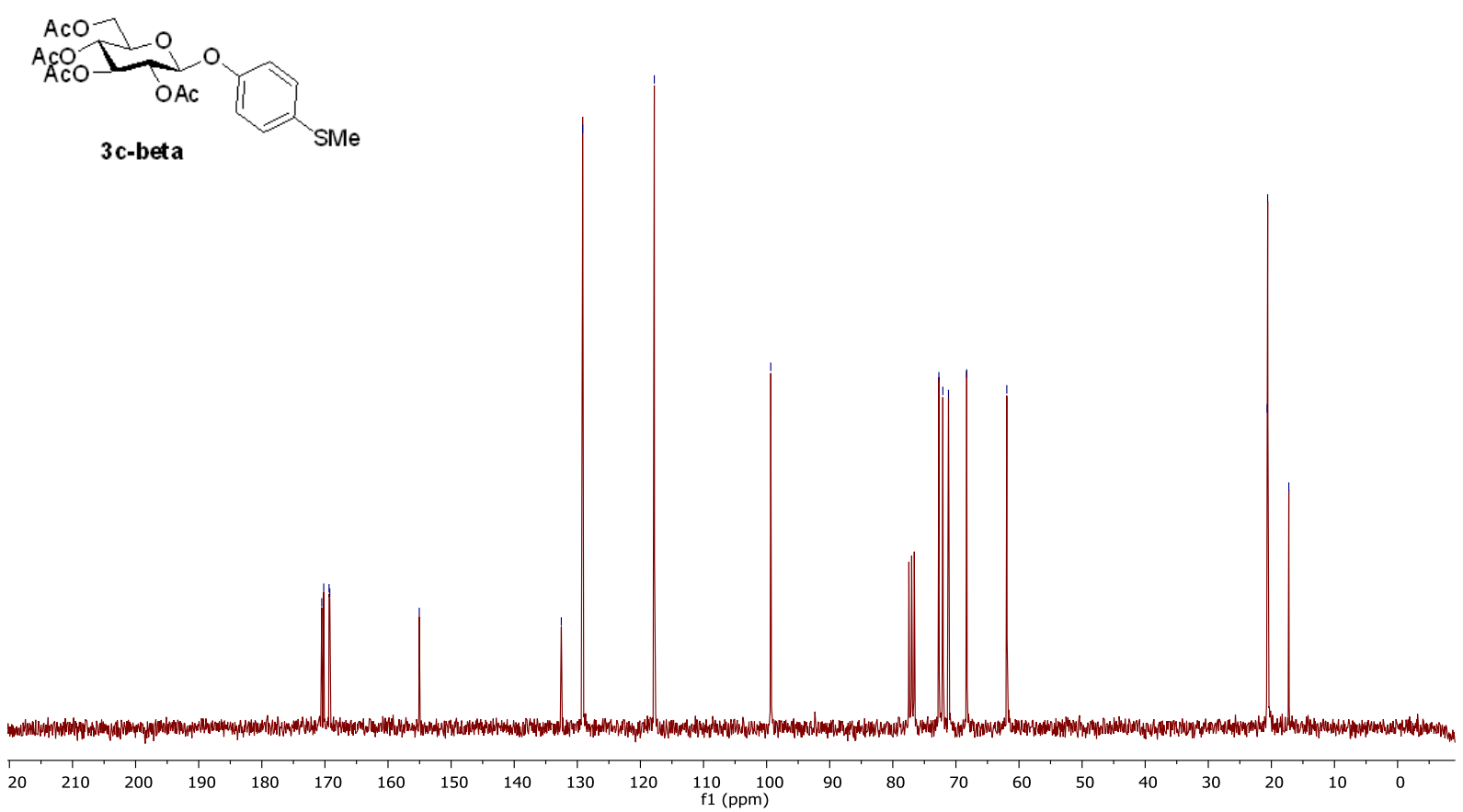


1-(4'-vinyl)-phenyl-2,3,4,6-tetra-O-acetyl-a-D-glucopyranoside

人
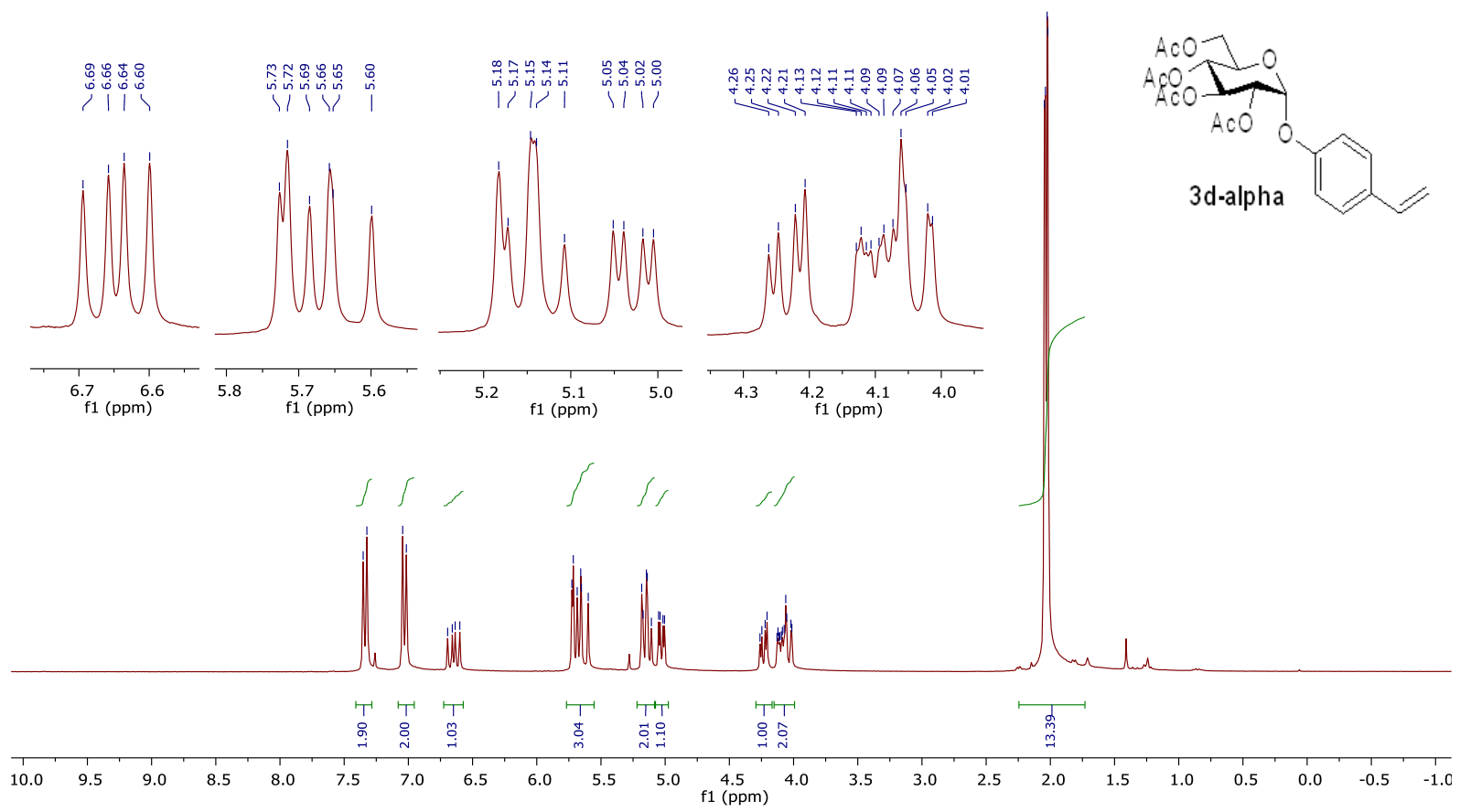

1-(4'-vinyl)-phenyl-2,3,4,6-tetra-O-acetyl-a-D-glucopyranoside

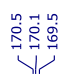

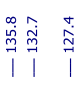

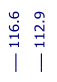

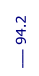

|com

ทิ่ง

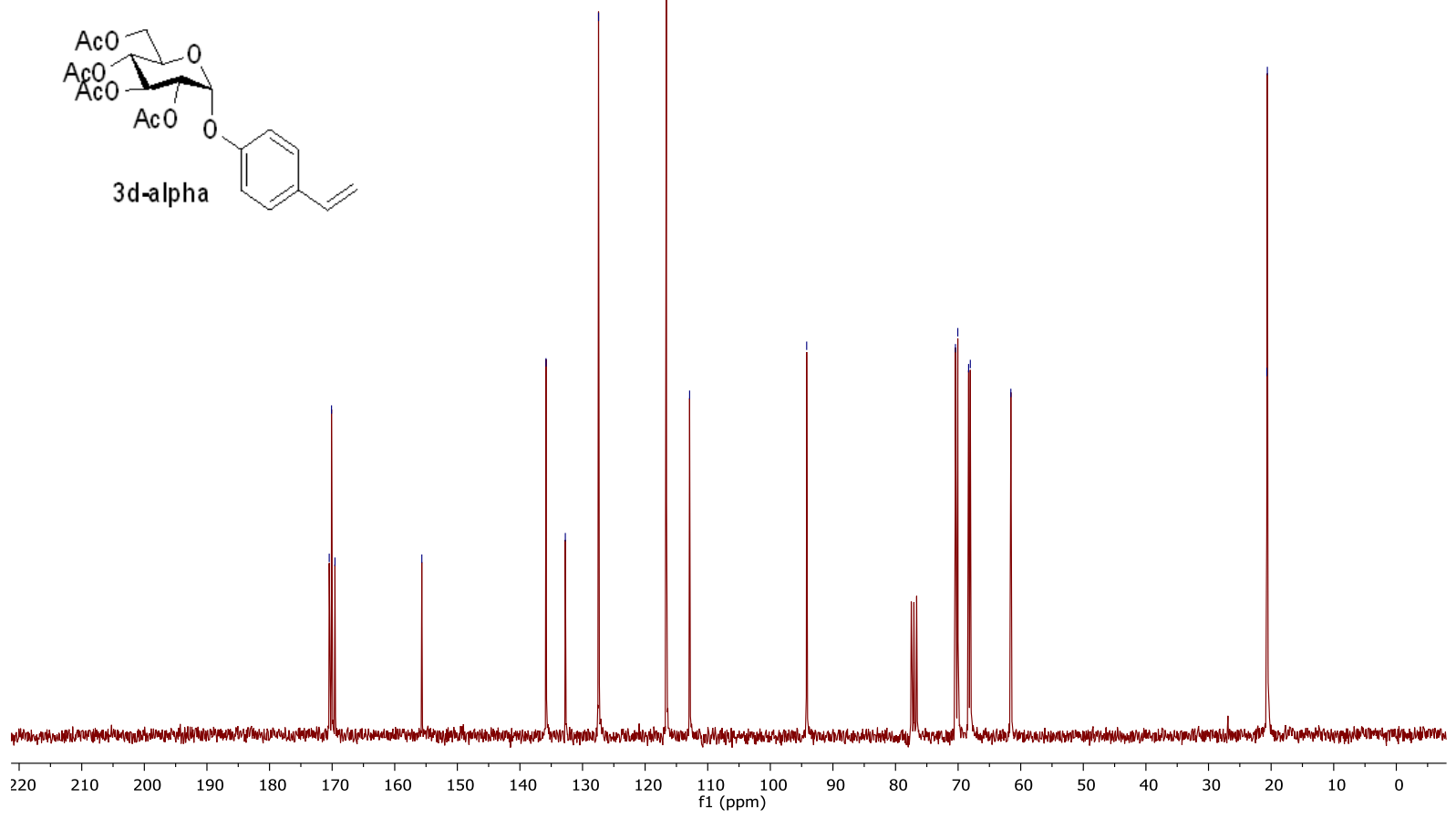

S10 

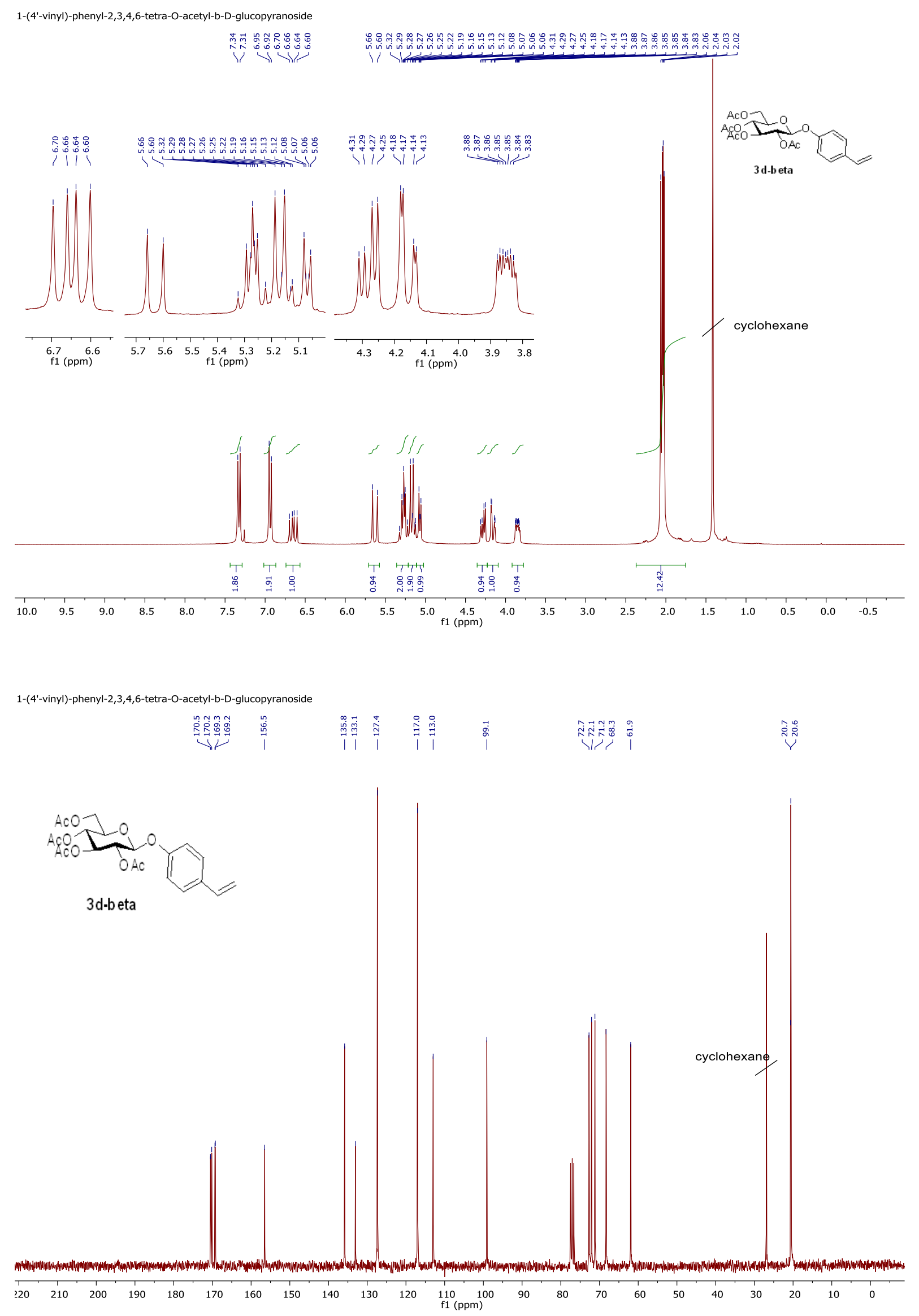

S11 


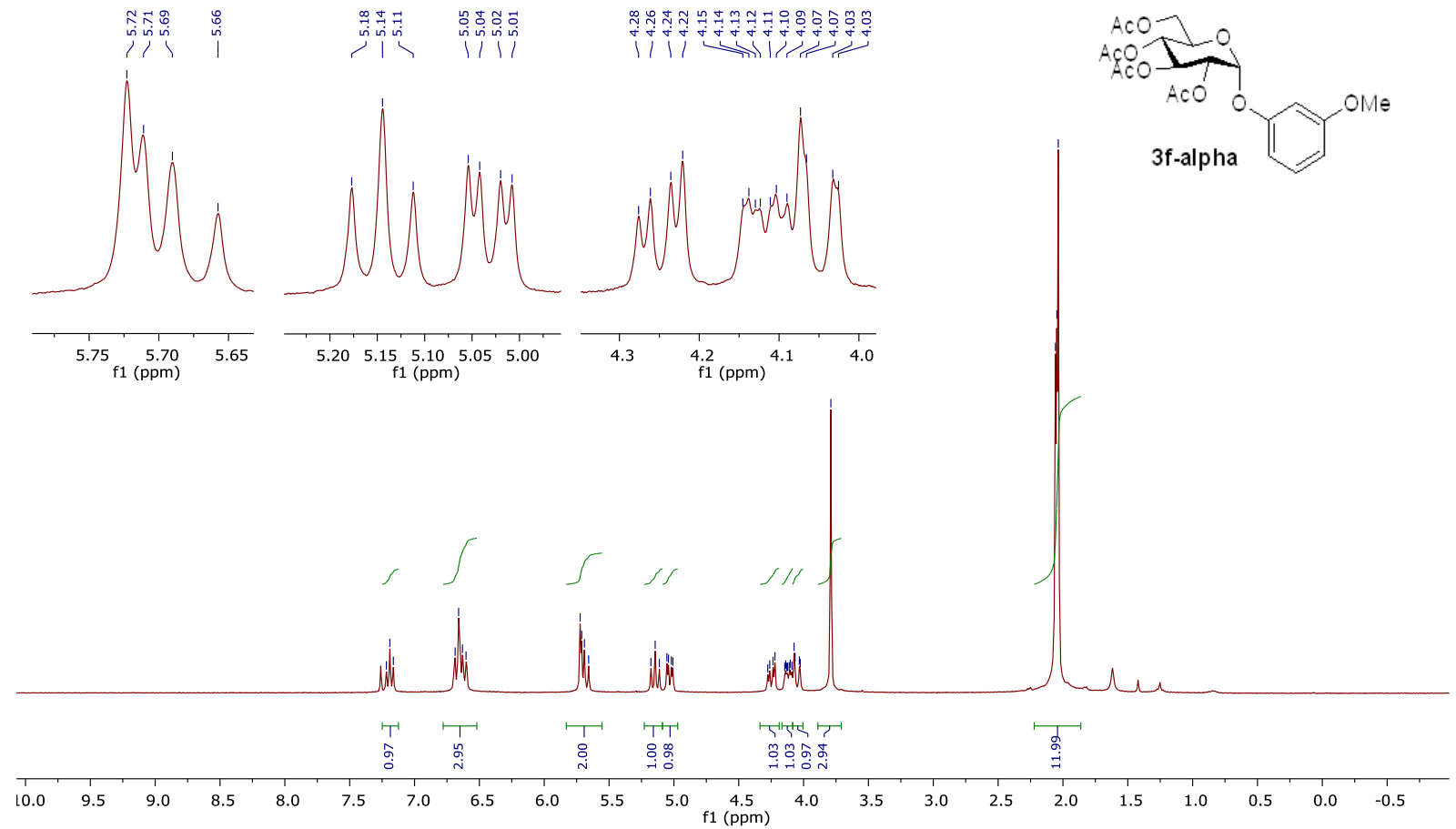

1-(3'-Methoxy)-2,3,4,6-tetra-O-acetyl-a-D-glucopyranoside

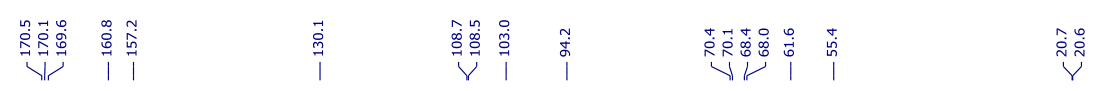
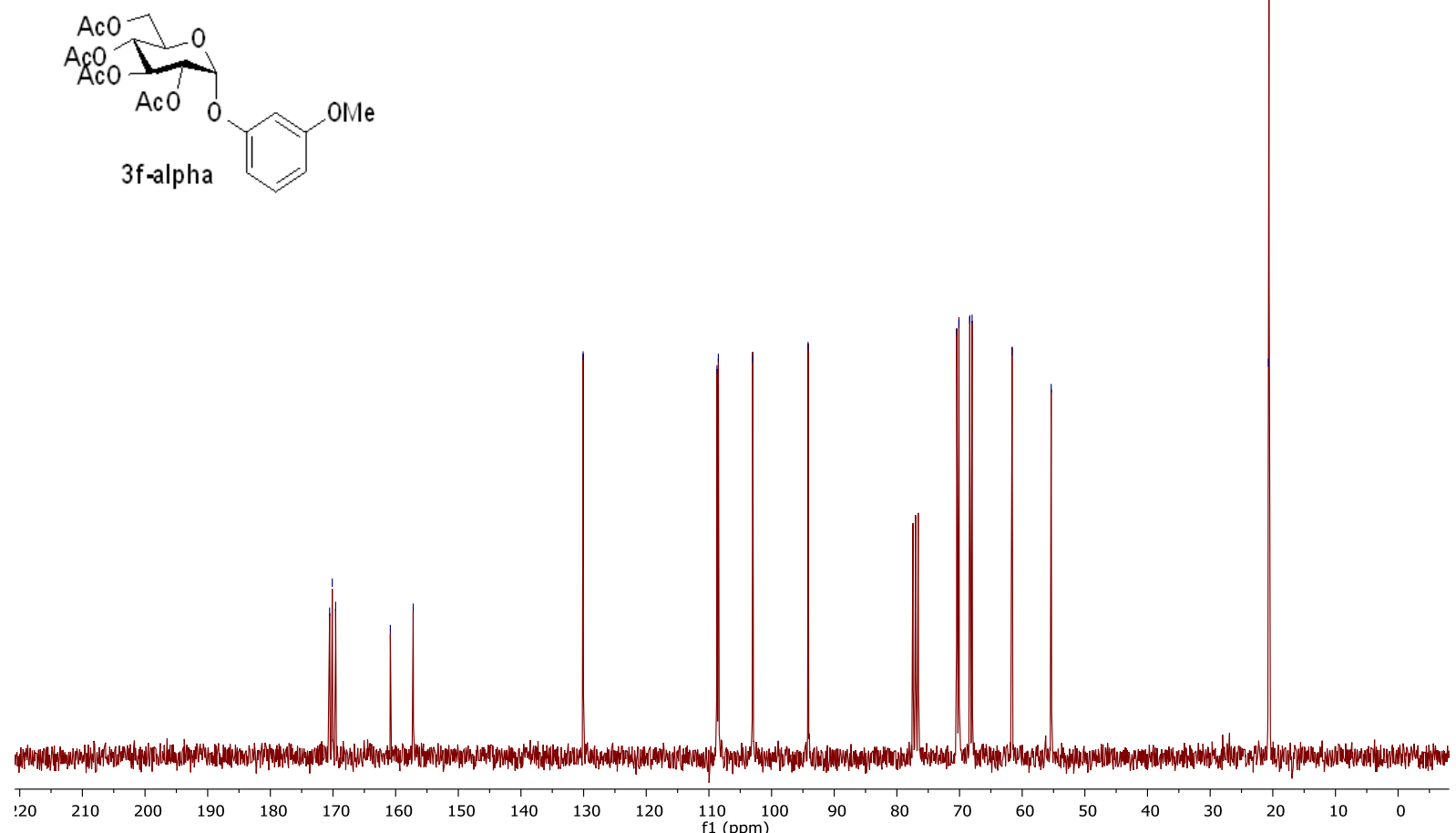


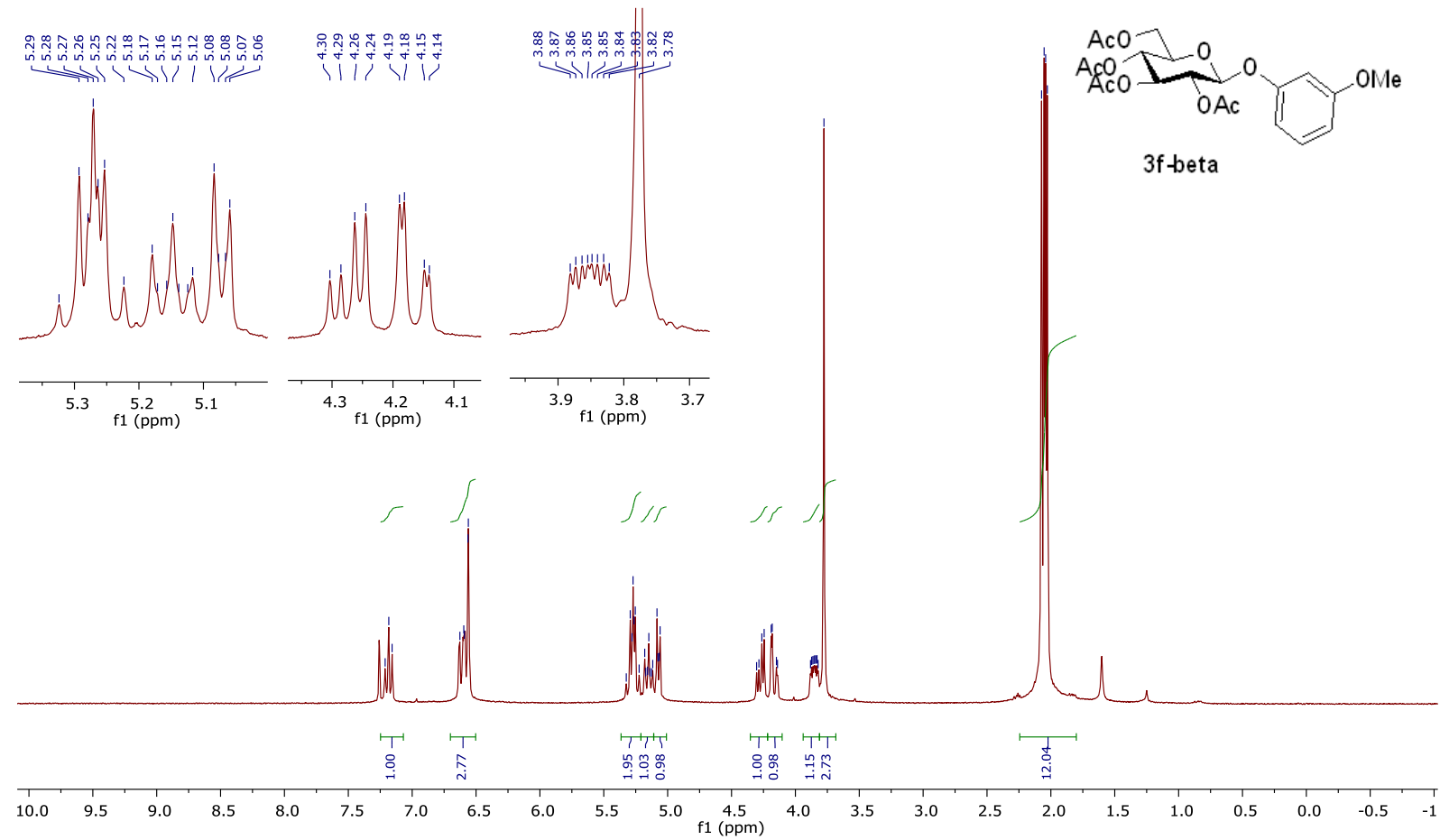

1-(3'-Methoxy)-2,3,4,6-tetra-O-acetyl-b-D-glucopyranoside

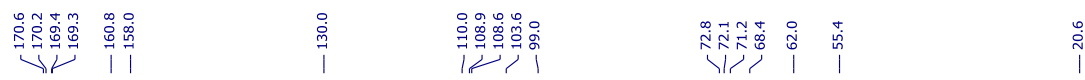

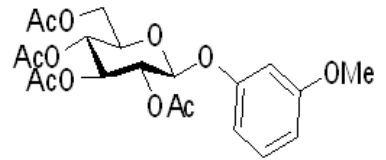

$3 f$ beta

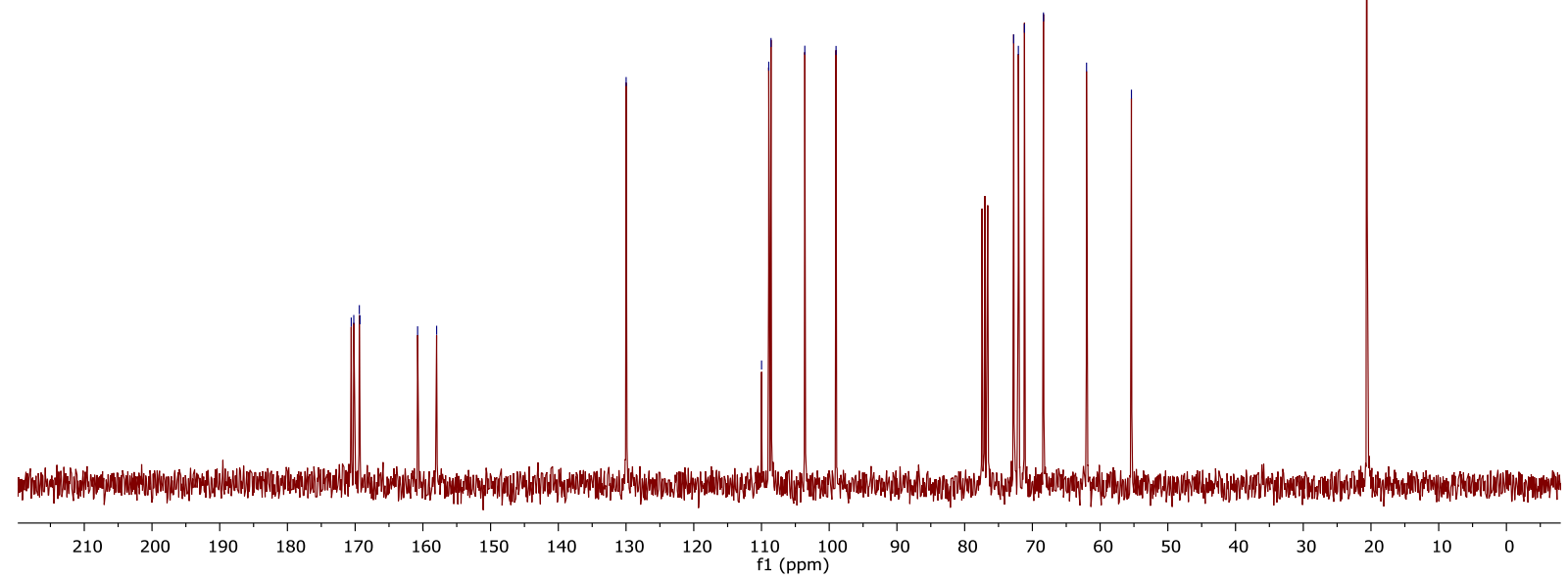




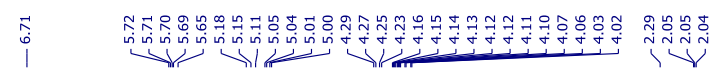

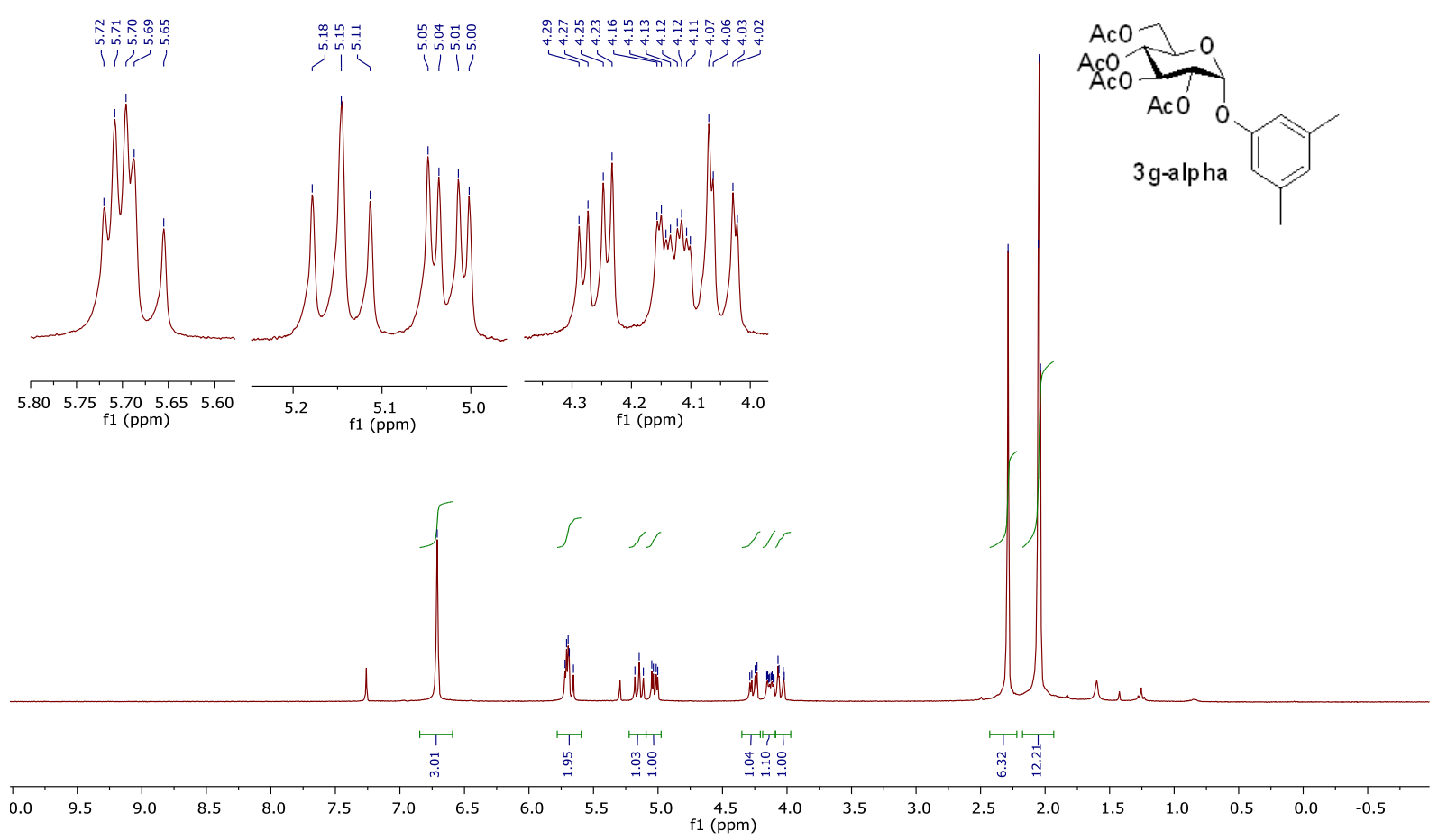

1-(3',5'-dimethyl)-phenyl-2,3,4,6-tetra-O-acetyl-a-D-glucopyranoside

象

$\stackrel{1}{\overrightarrow{0}}$

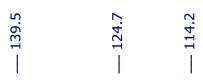

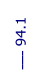

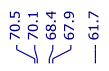

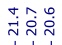

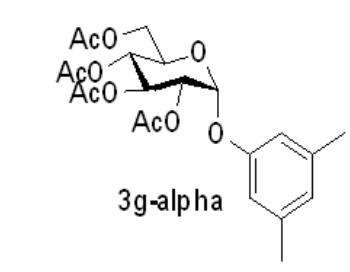

$\mathrm{S} 14$ 


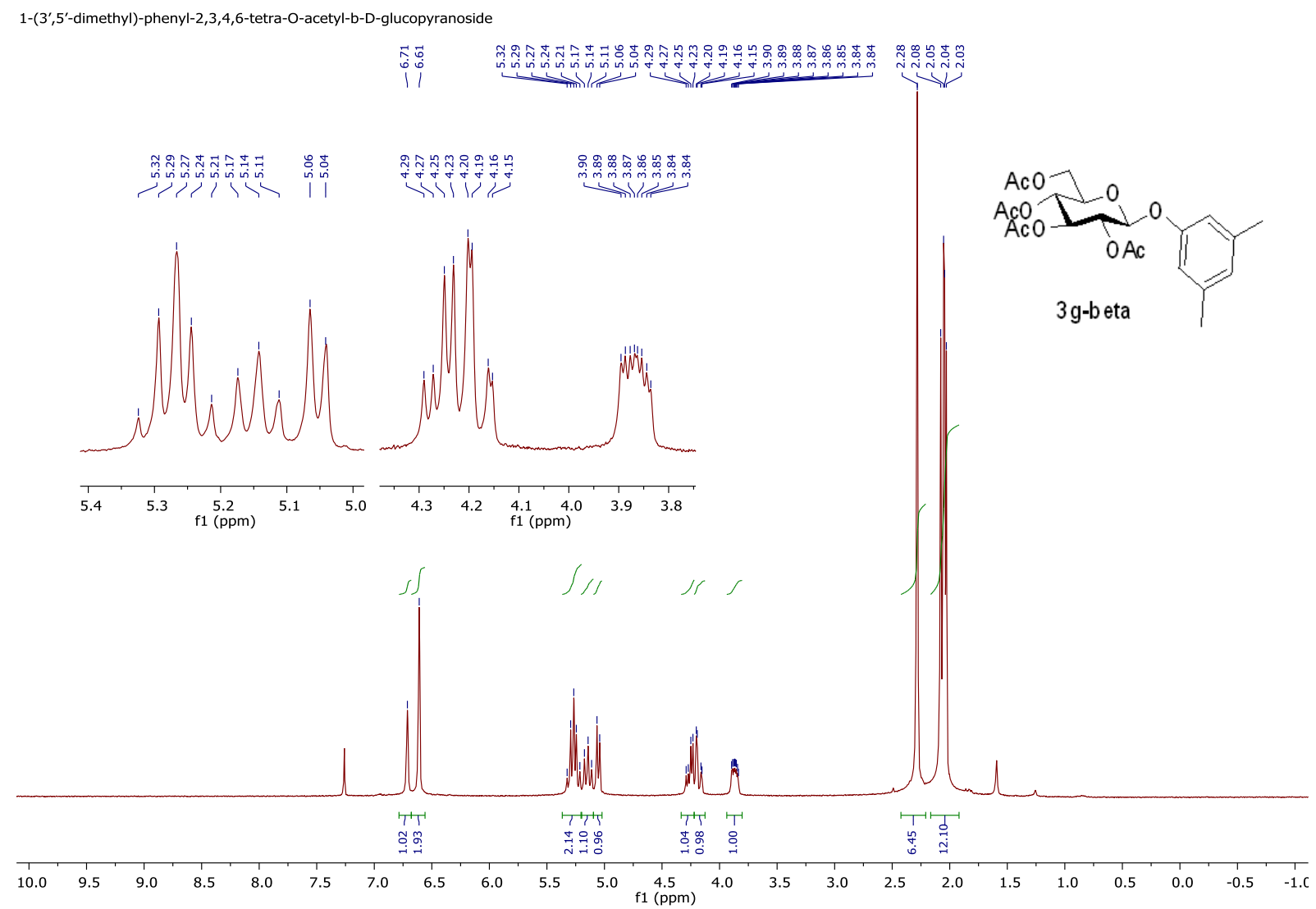

1-(3',5'-dimethyl)-phenyl-2,3,4,6-tetra-O-acetyl-b-D-glucopyranoside
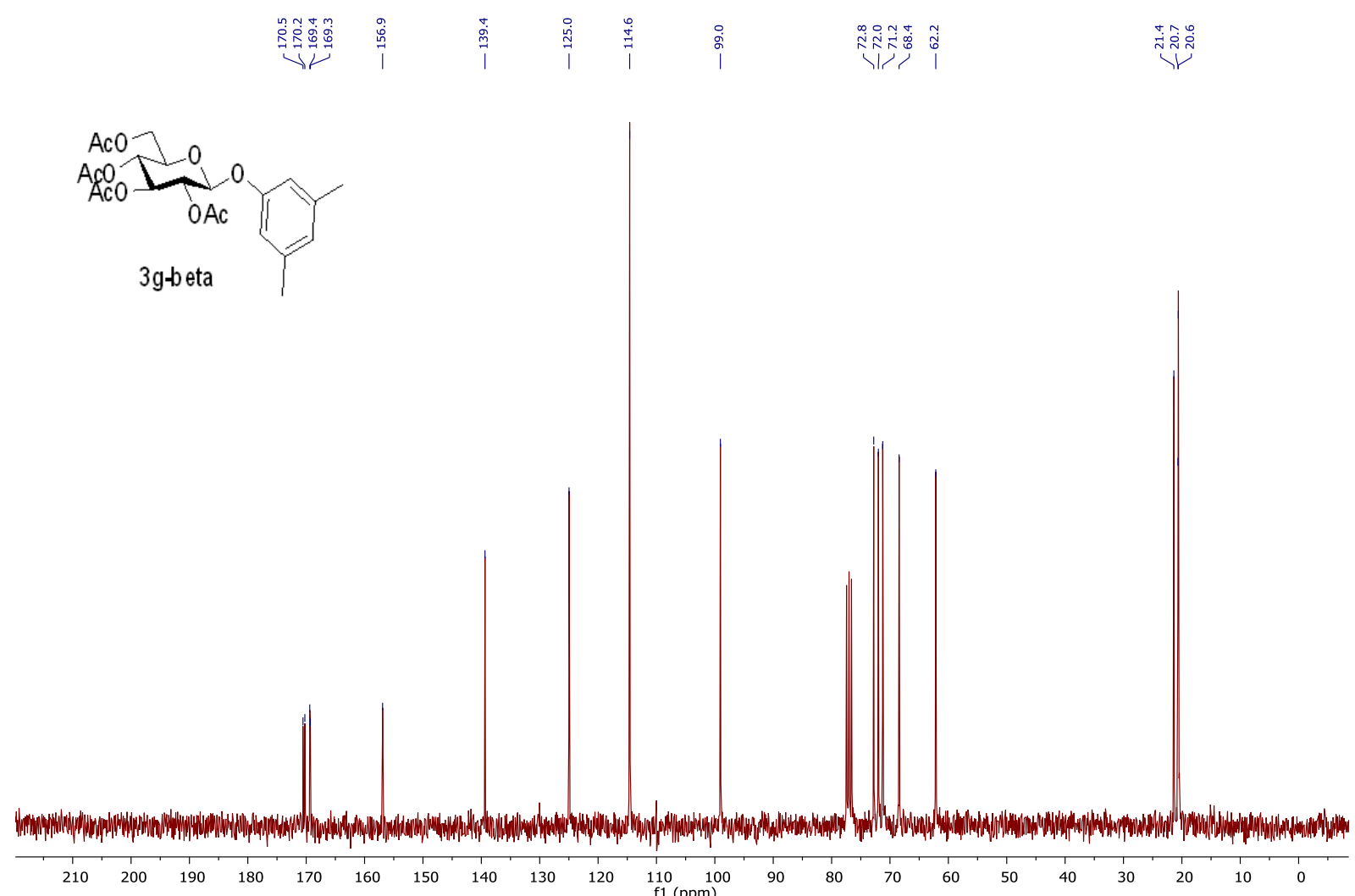


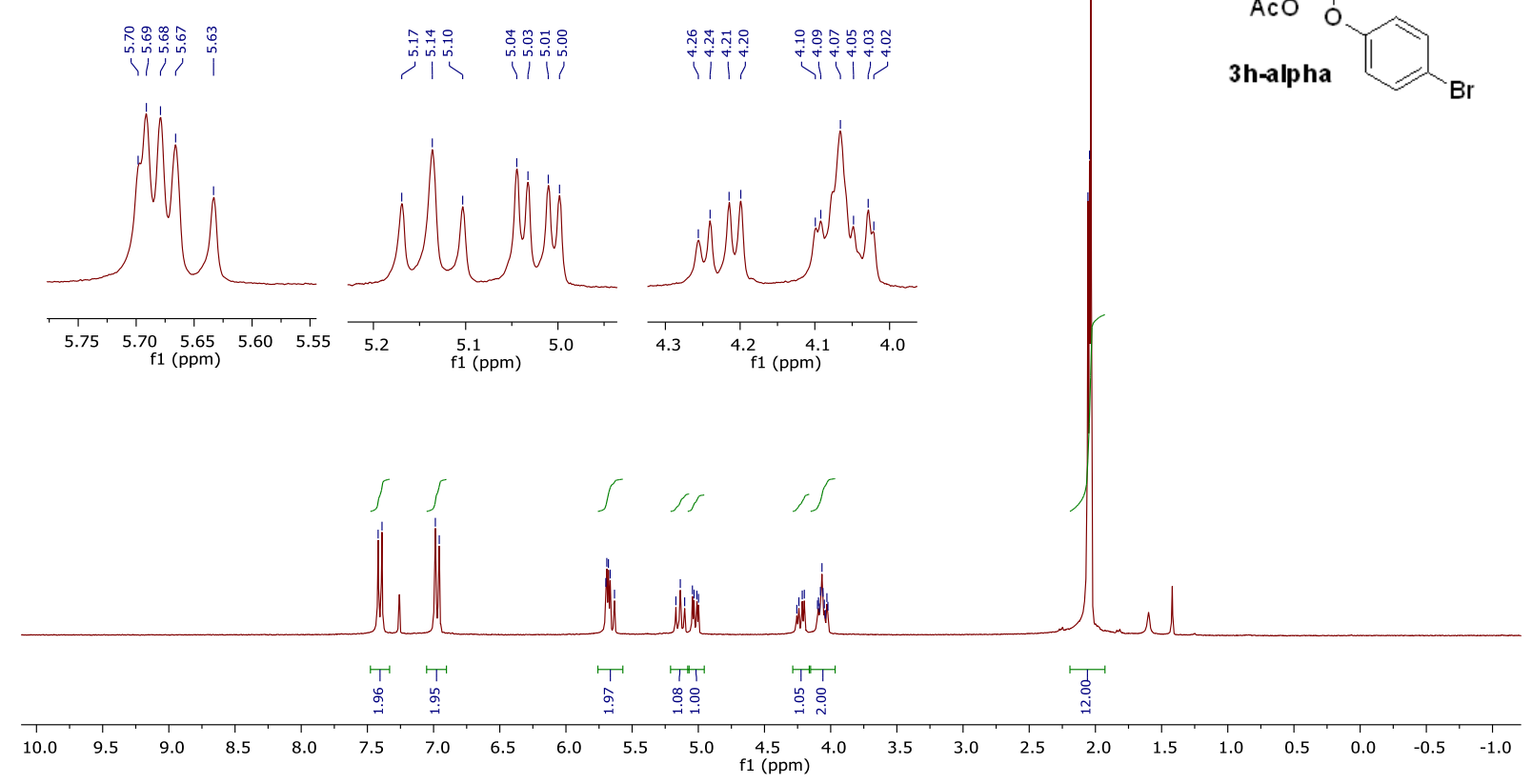

4'-Bromophenyl 2,3,4,6-tetra-O-acetyl-a-D-glucopyranoside

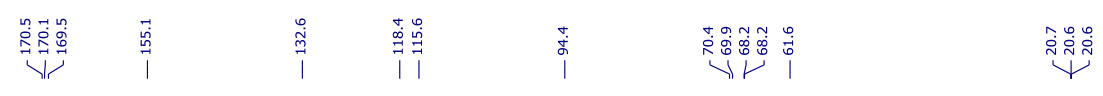
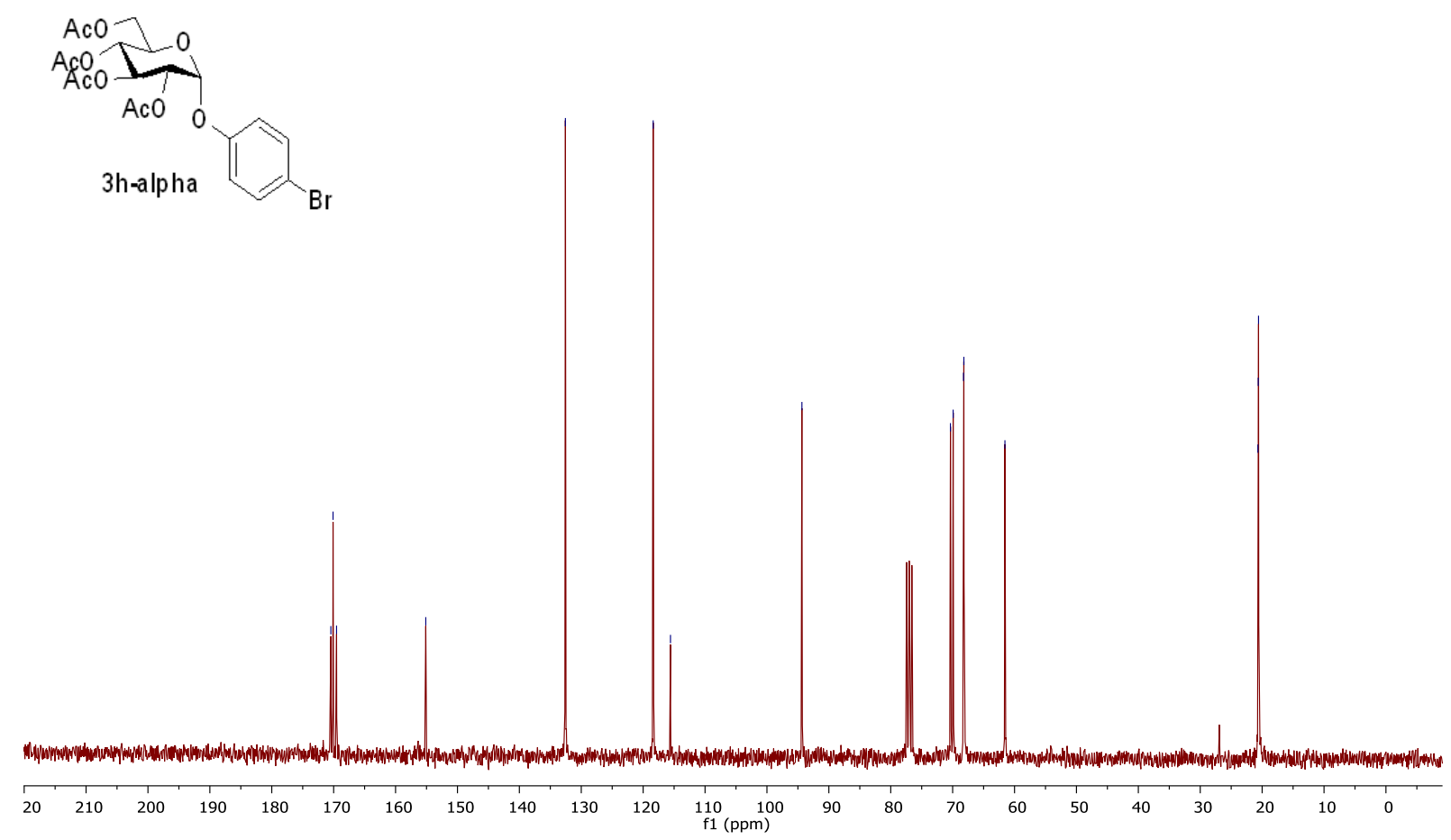


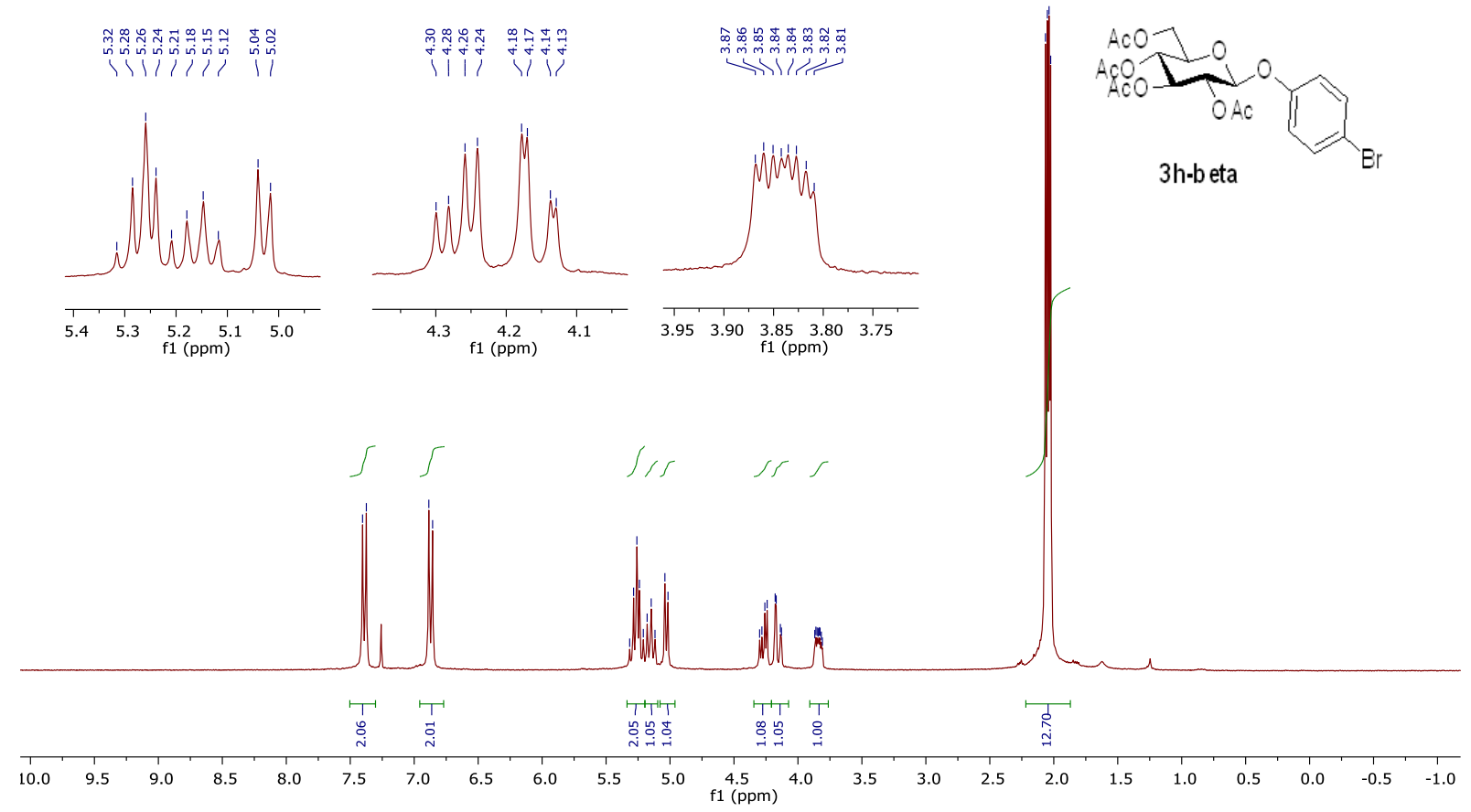

4'-Bromophenyl 2,3,4,6-tetra-O-acetyl-b-D-glucopyranoside

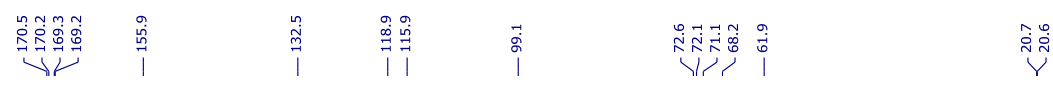

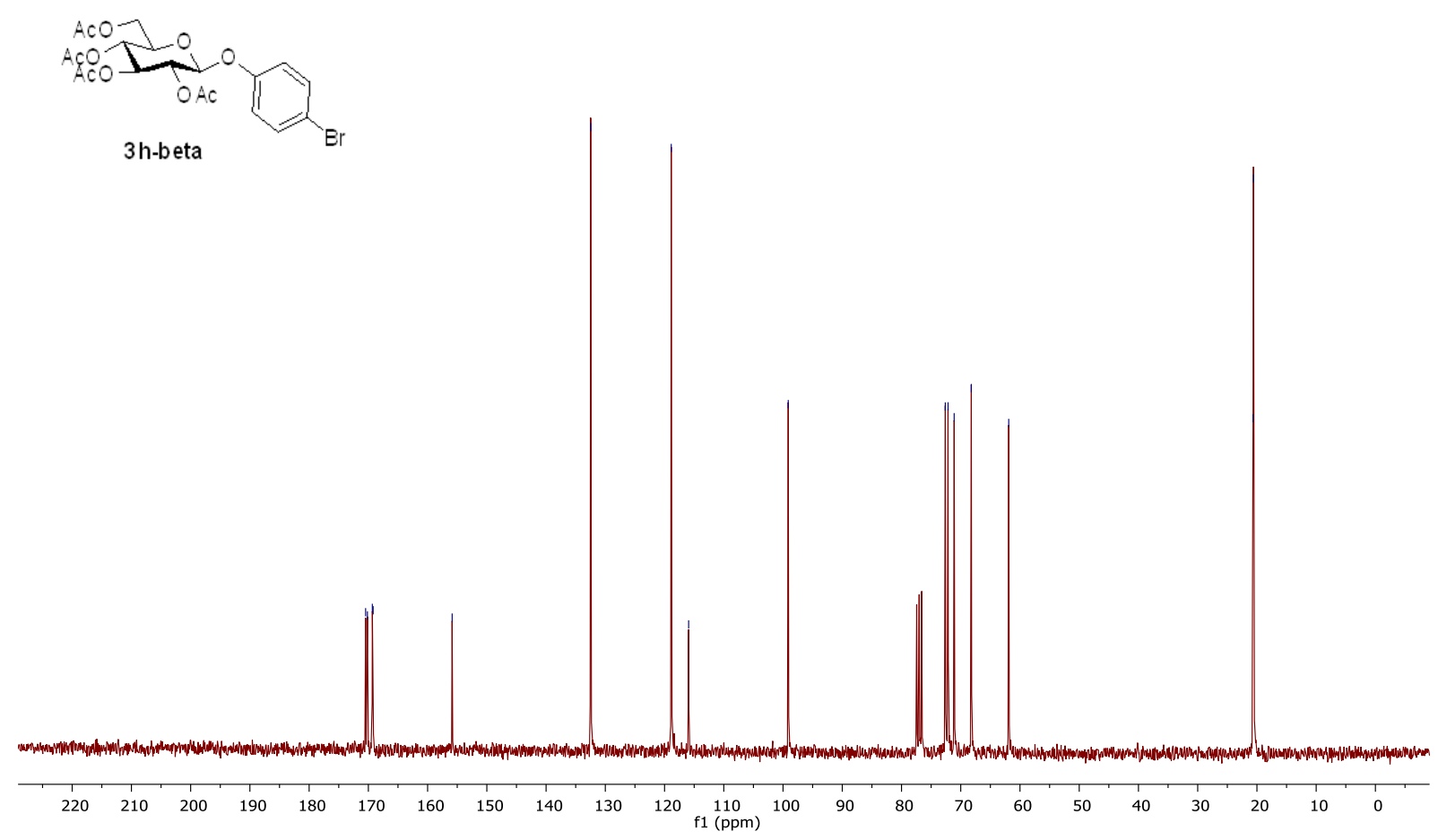


4'-Fluorophenyl 2,3,4,6-tetra-O-acetyl-a-D-glucopyranoside

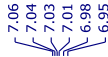

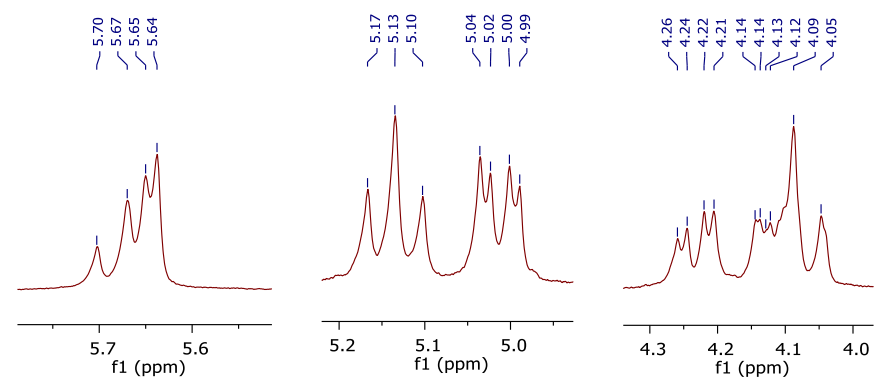

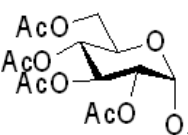

3i-alpha
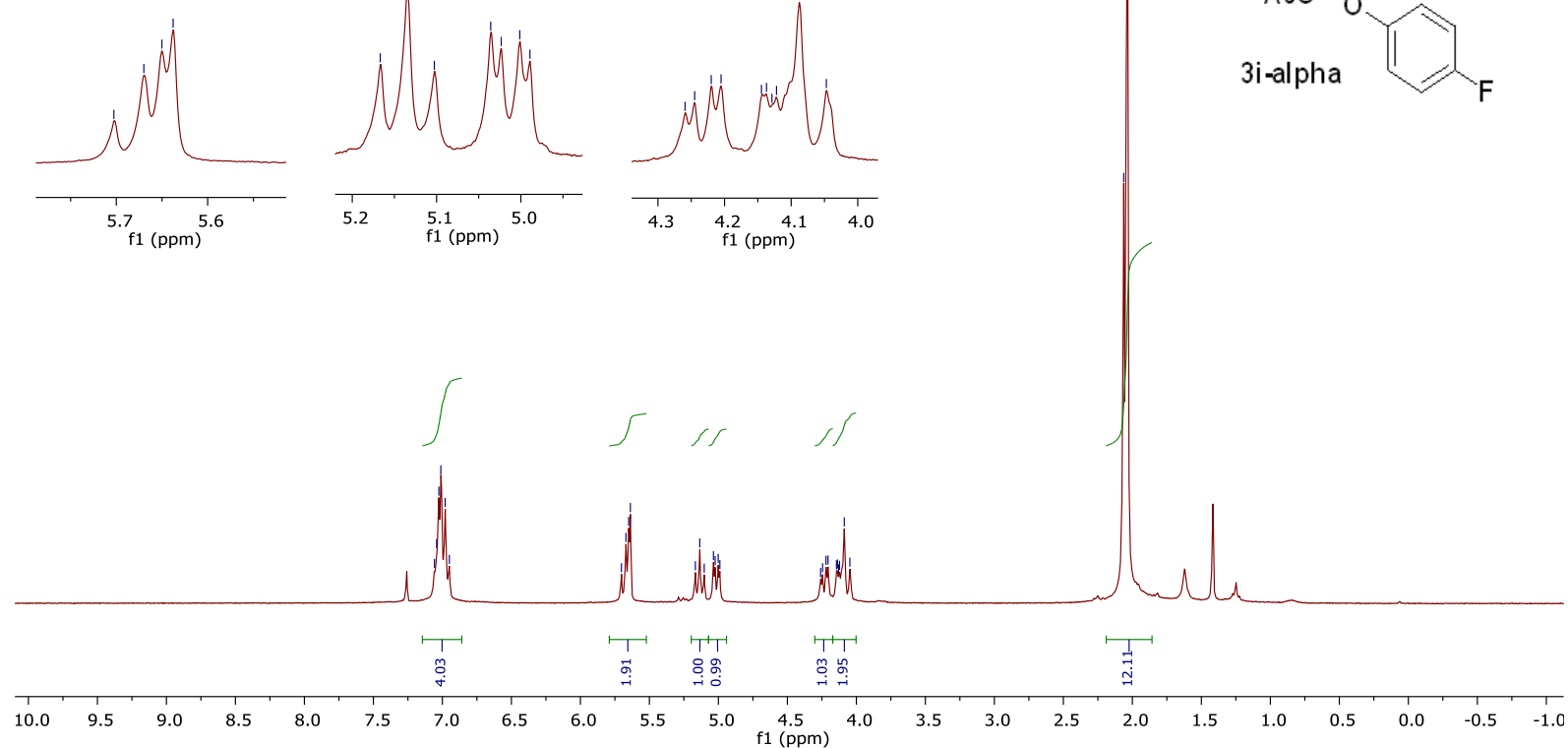

4'-Fluorophenyl 2,3,4,6-tetra-O-acetyl-a-D-glucopyranoside

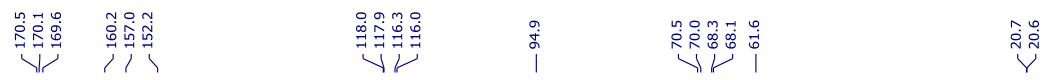
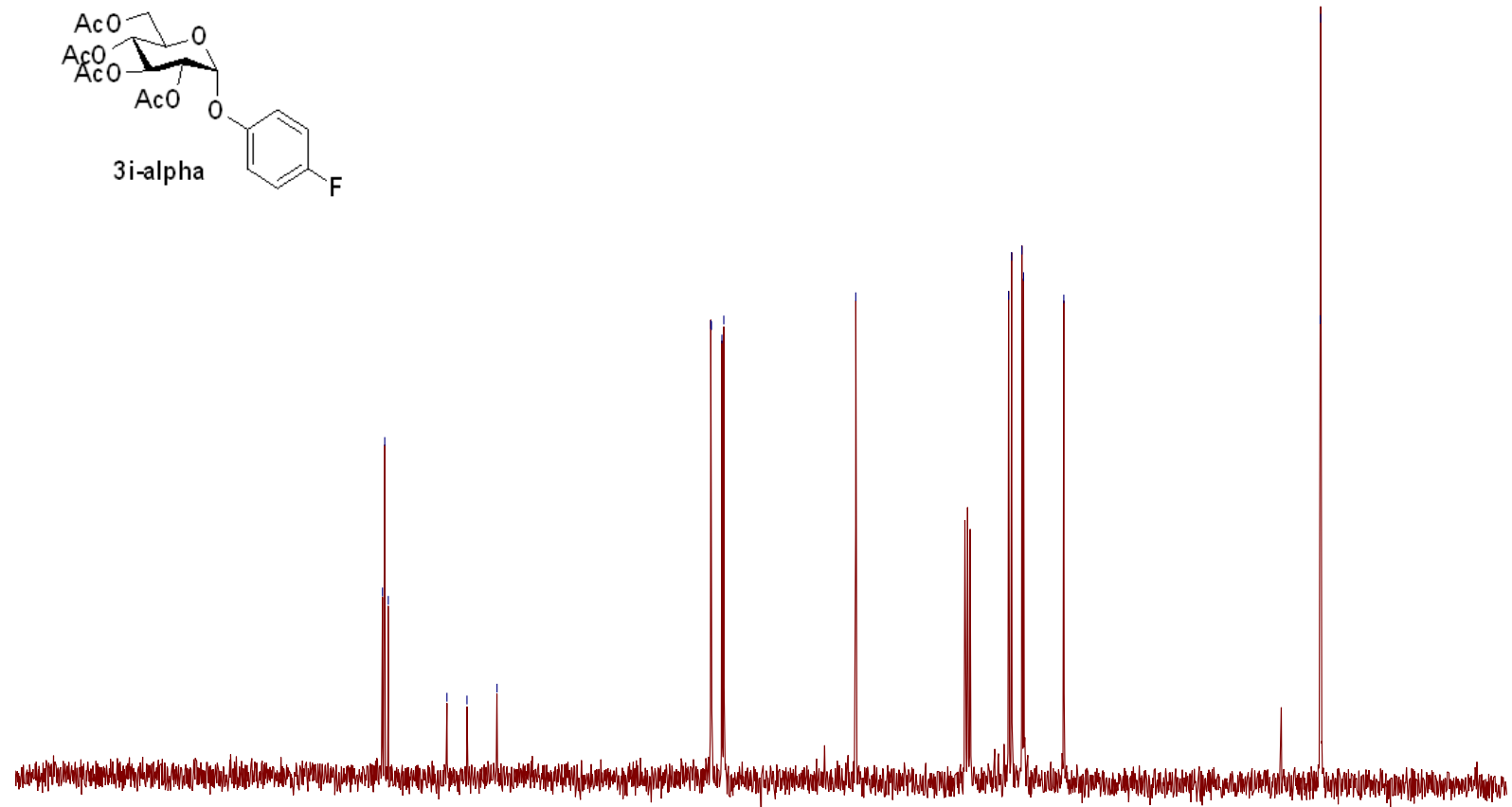

$\begin{array}{lllllllllllllllllllllll}220 & 210 & 200 & 190 & 180 & 170 & 160 & 150 & 140 & 130 & 120 & \underset{f 1}{110} & 100 & 90 & 80 & 70 & 60 & 50 & 40 & 30 & 20 & 10 & 0\end{array}$ 
4'-Fluorophenyl 2,3,4,6-tetra-O-acetyl-b-D-glucopyranoside

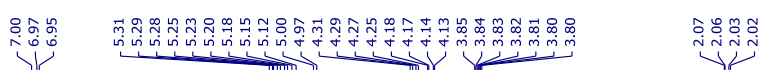
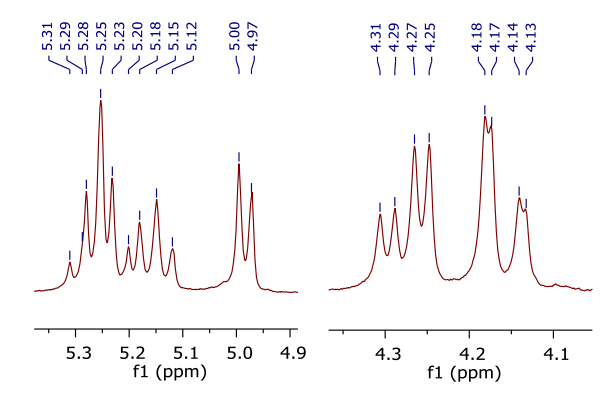

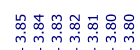

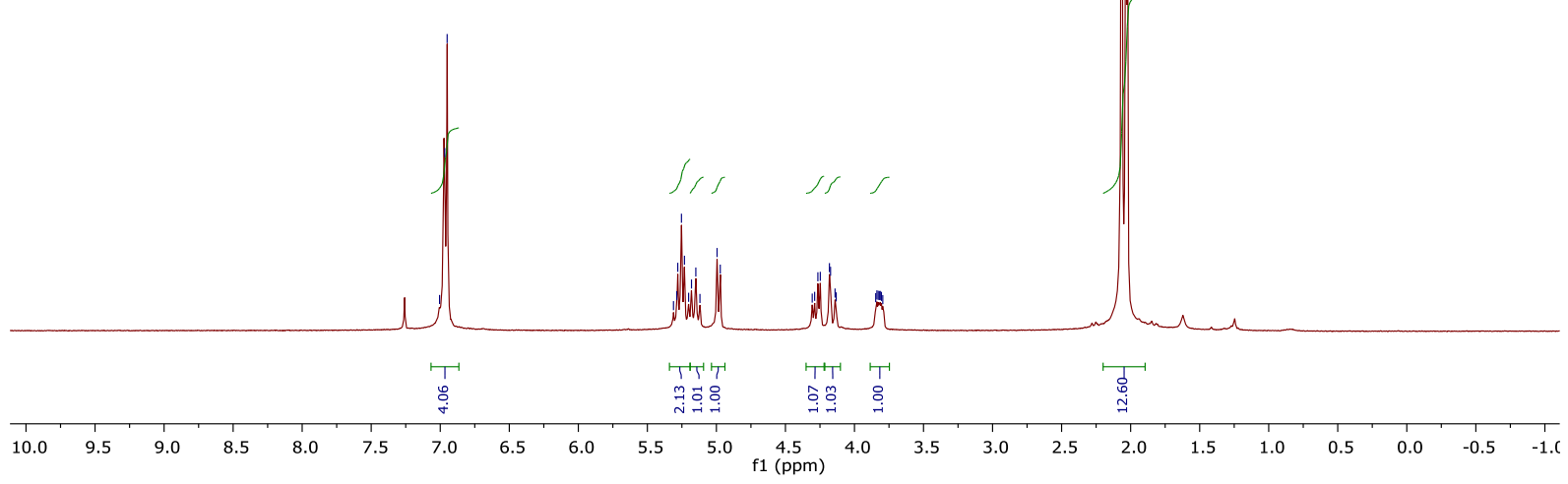

4'-Fluorophenyl 2,3,4,6-tetra-O-acetyl-b-D-glucopyranoside

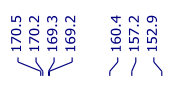

ע山

ò่

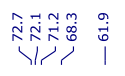

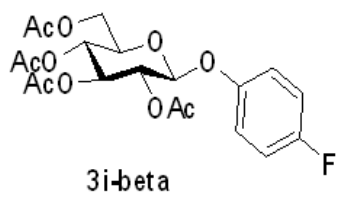

$3 i$ beta
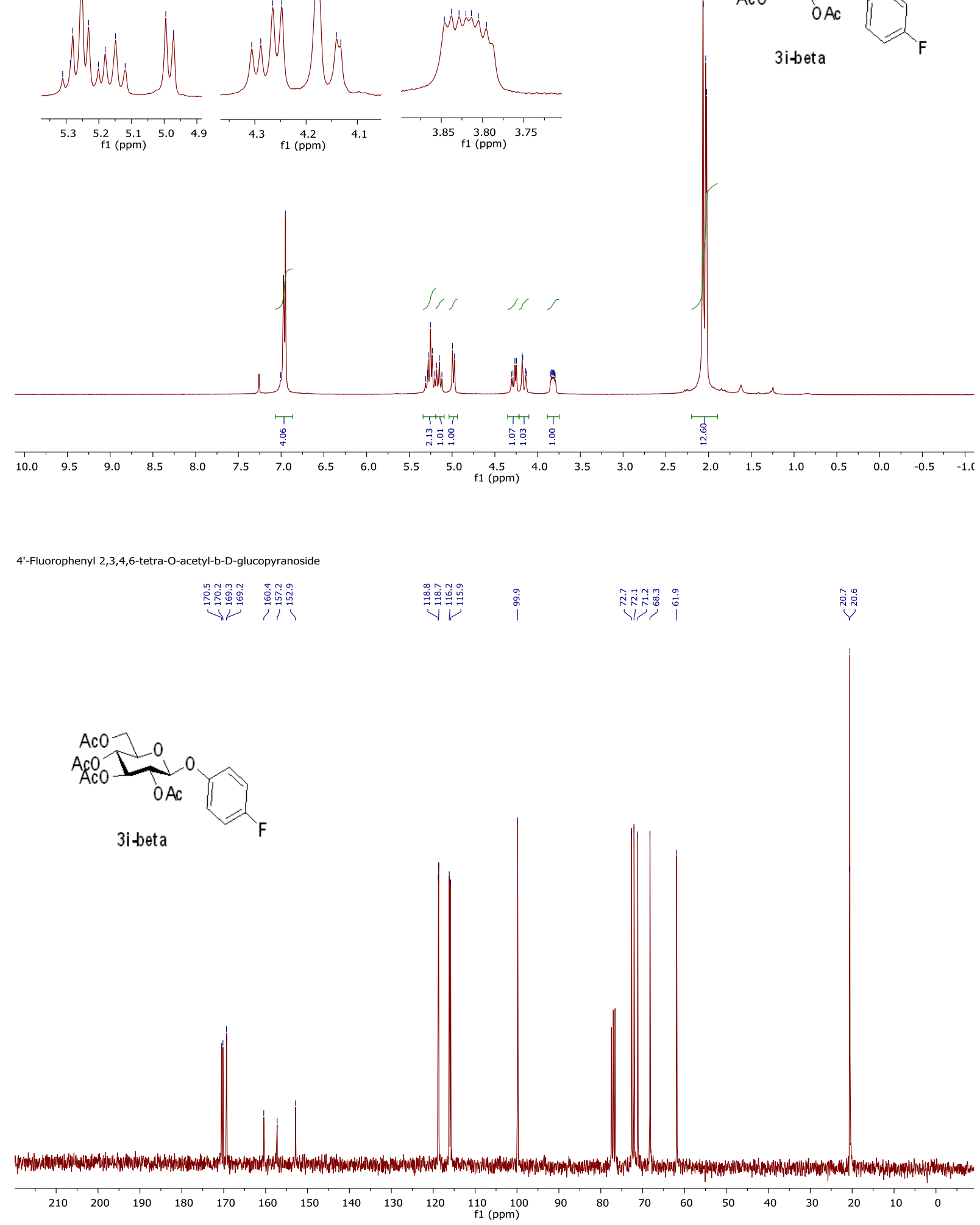

S19 
1-(4'-Trifluoromethyl)phenyl-2,3,4,6-tetra-O-acetyl-a-D-glucopyranoside

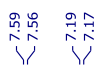

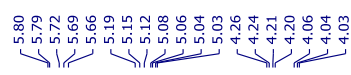

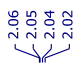
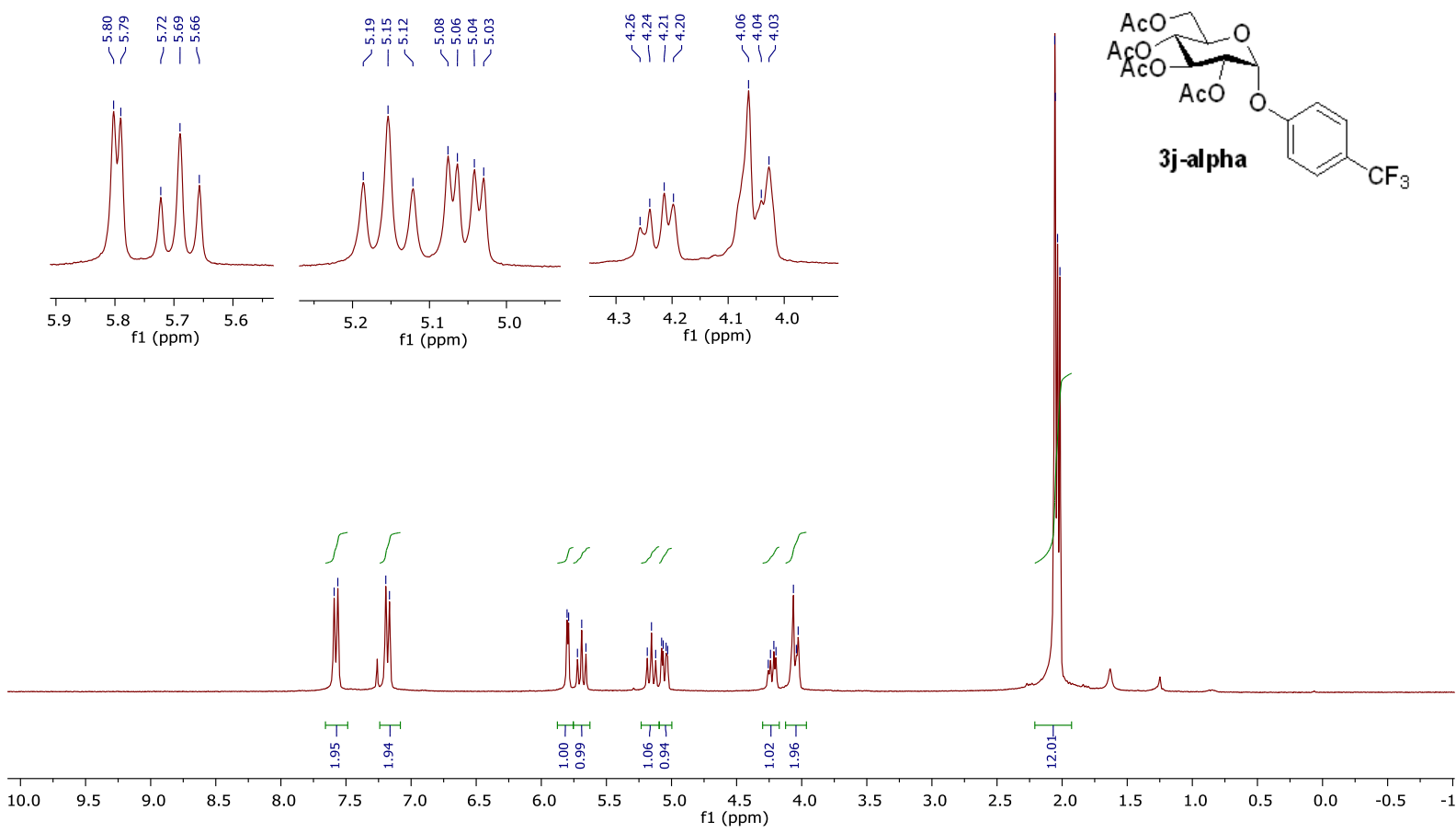

1-(4'-trifluoromethoxy)-phenyl-2,3,4,6-tetra-O-acetyl-a-D-glucopyranoside

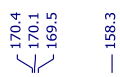

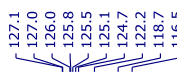

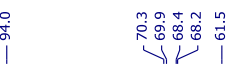

บับ

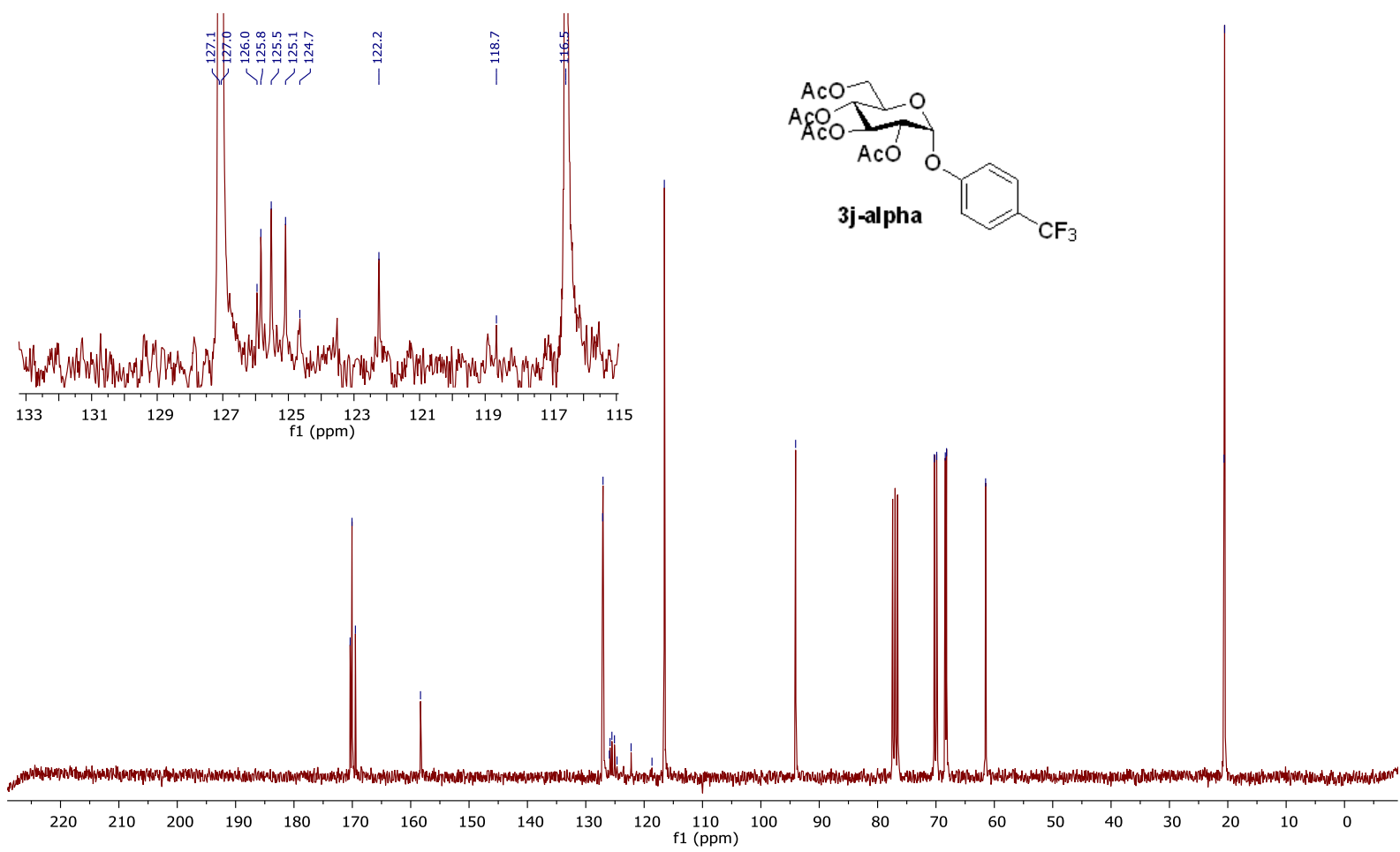

S20 
(4'-Trifluoromethyl)phenyl 2,3,4,6-tetra-O-acetyl-b-D-glucopyranoside

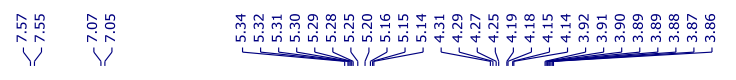

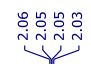
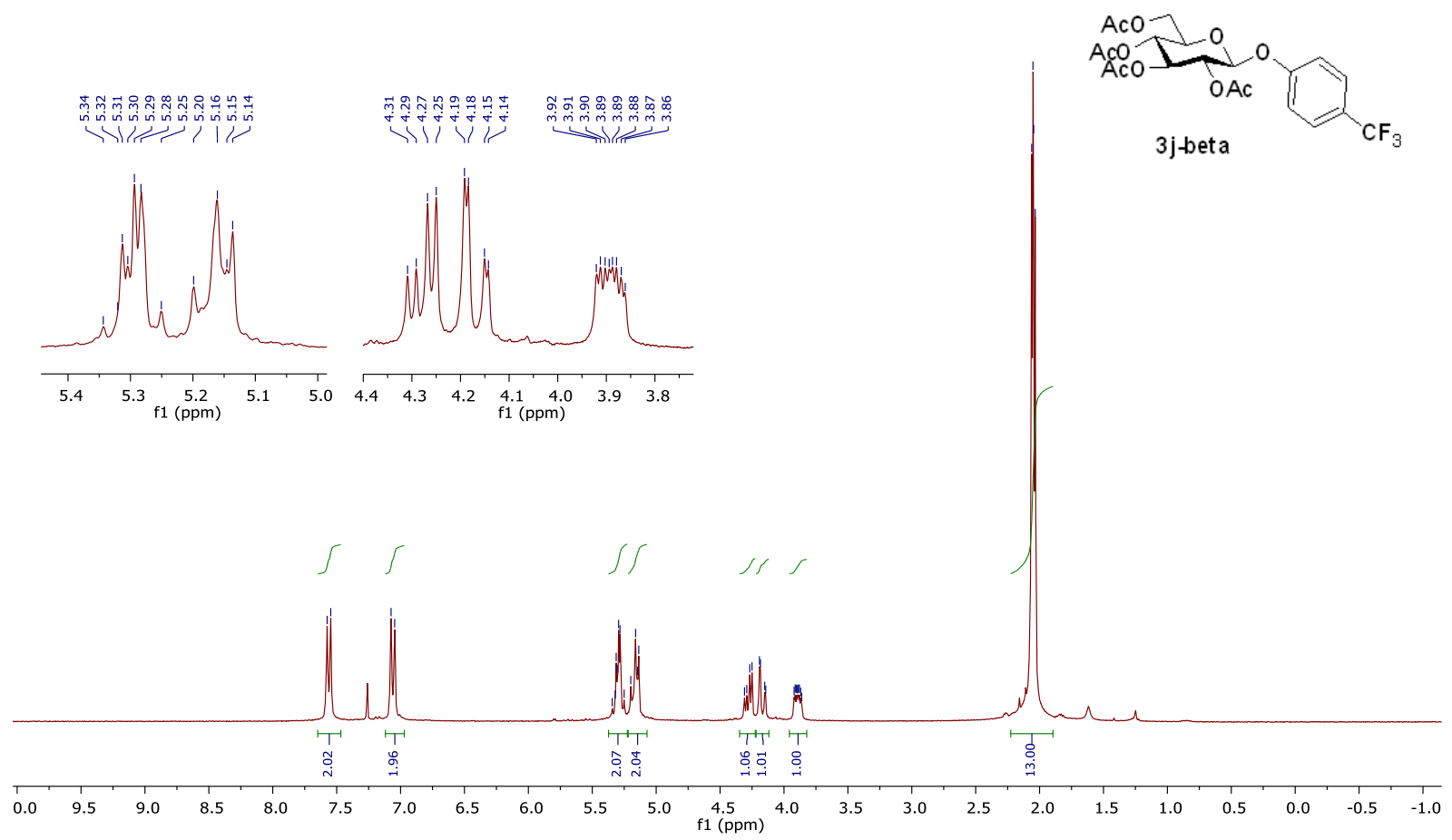

1-(4'-Trifluoromethyl)-phenyl-2,3,4,6-tetra-O-acetyl-b-D-glucopyranoside

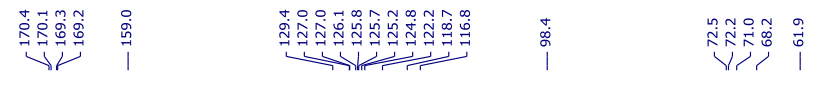

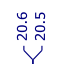

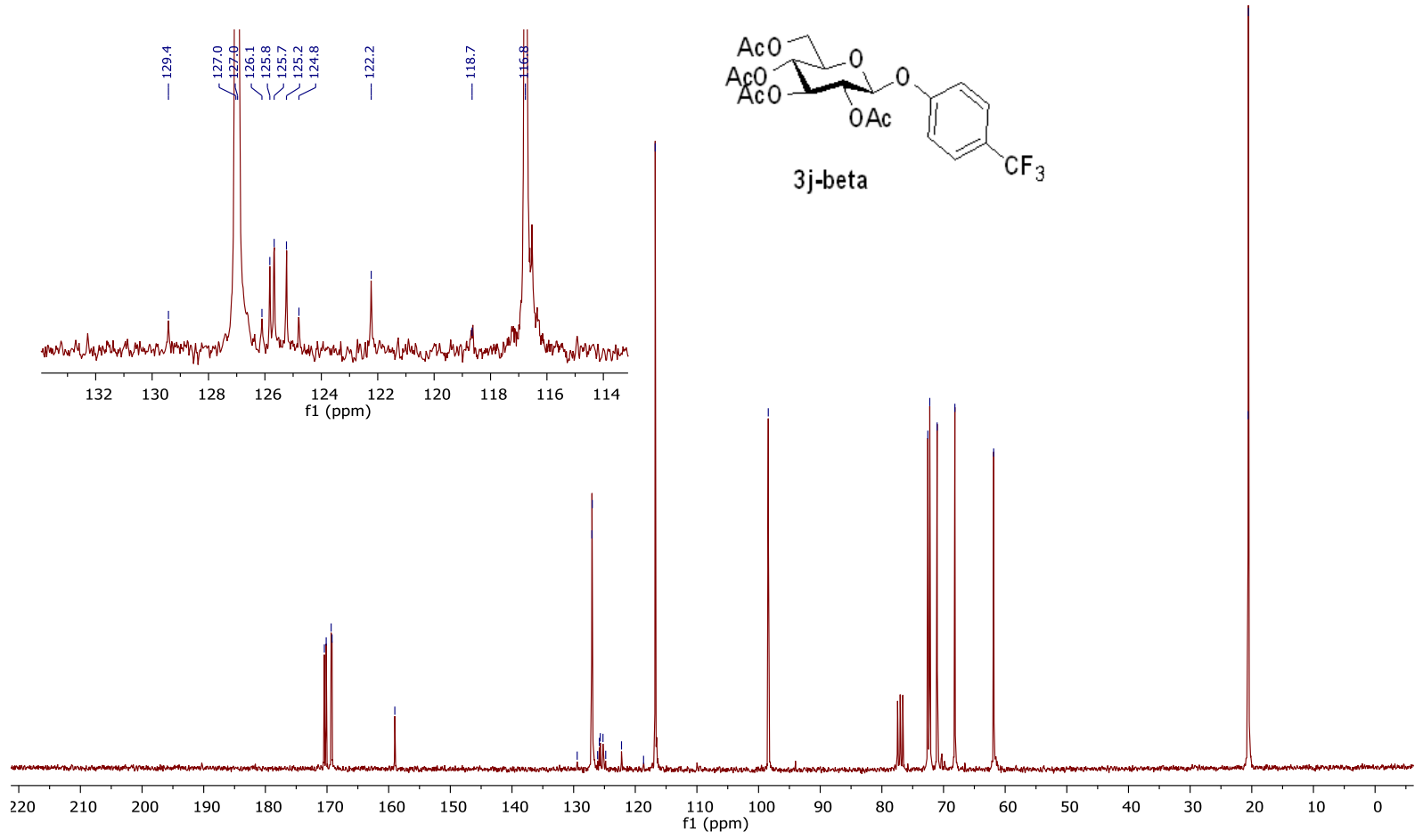

S21 

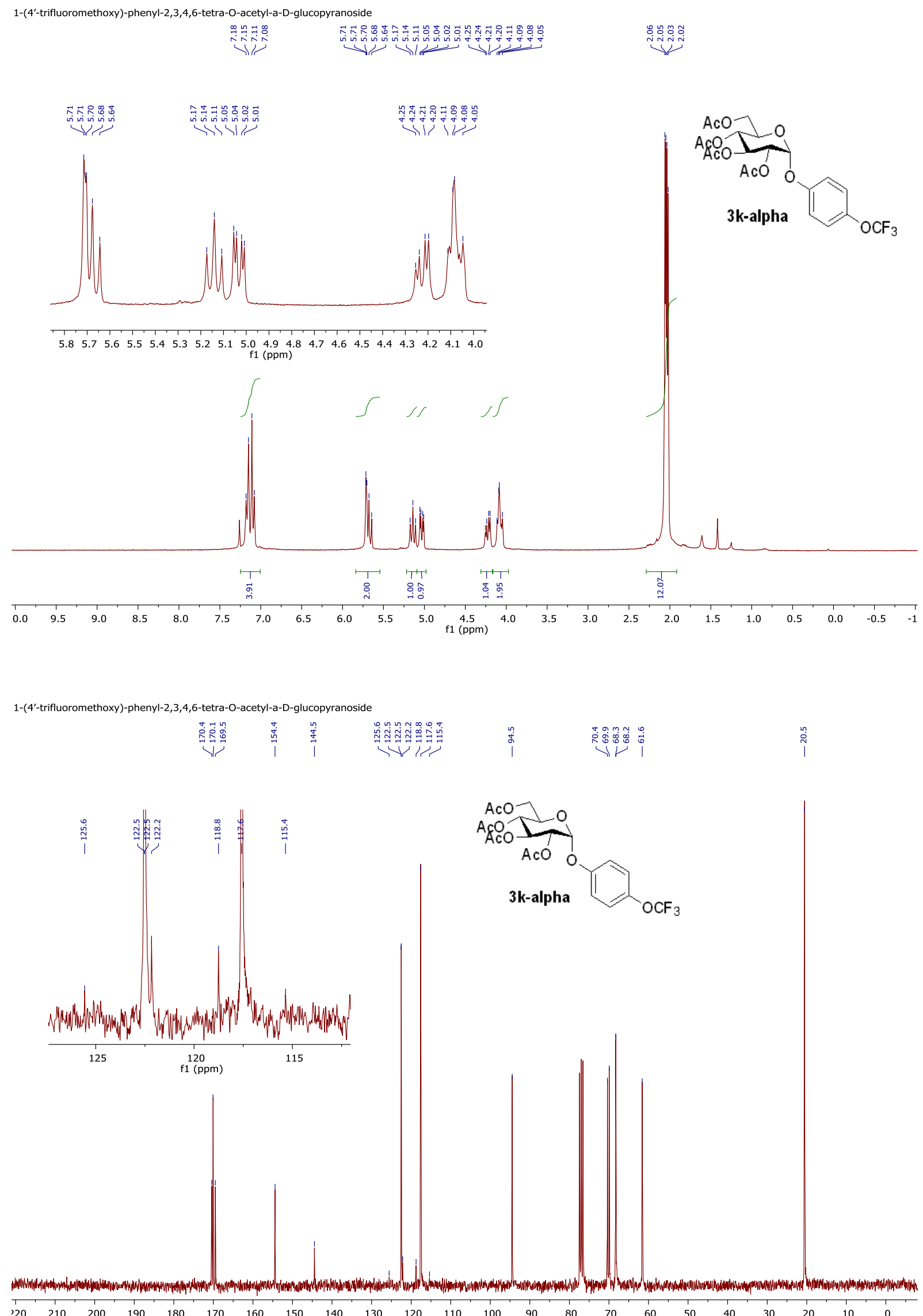

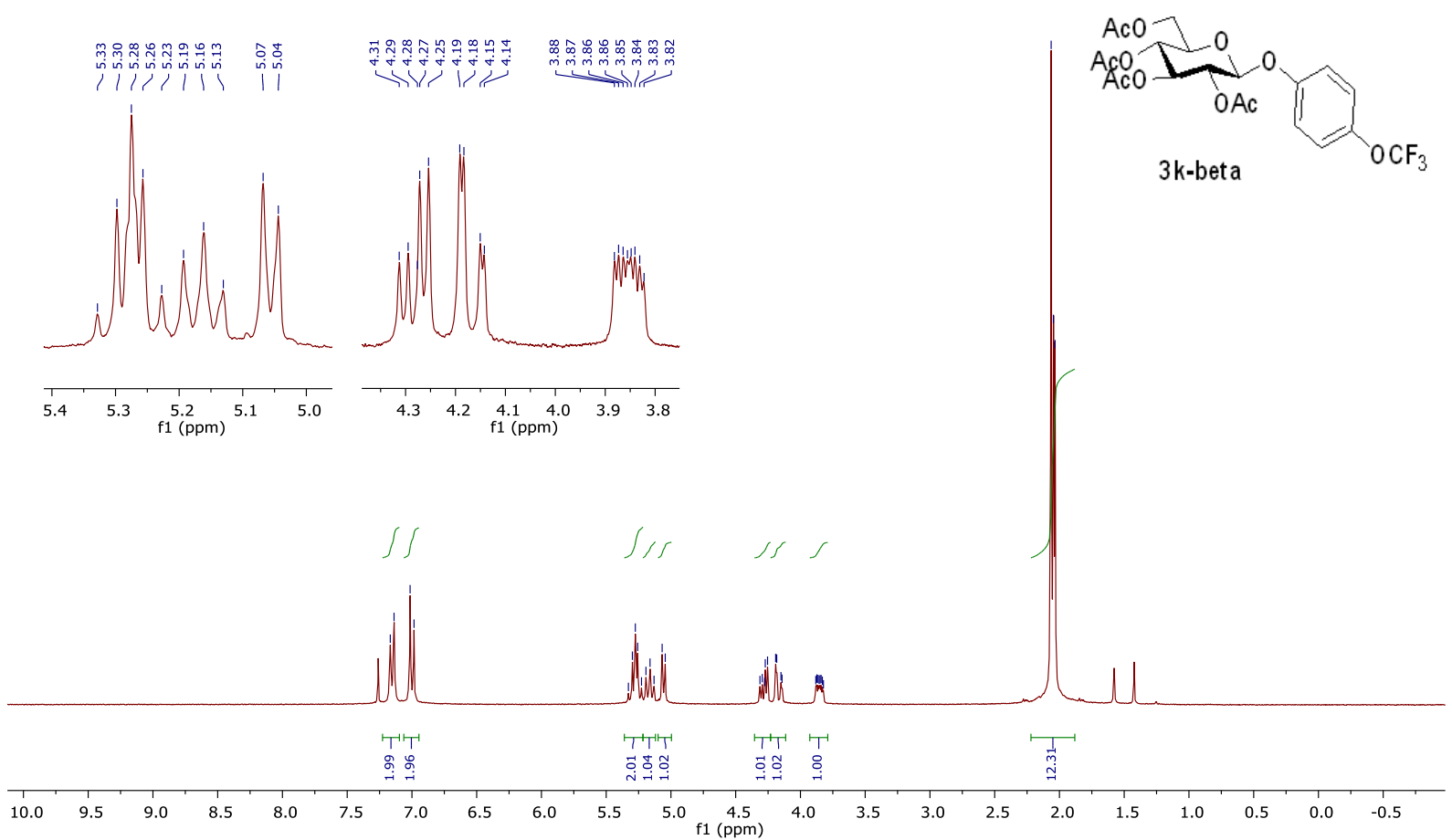

1-(4'-trifluoromethoxy)-phenyl-2,3,4,6-tetra-O-acetyl-b-D-glucopyranoside

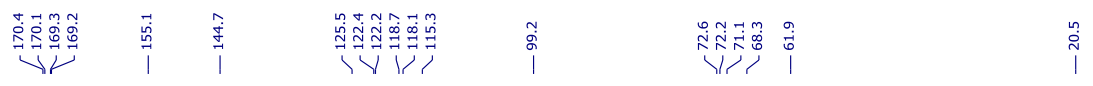
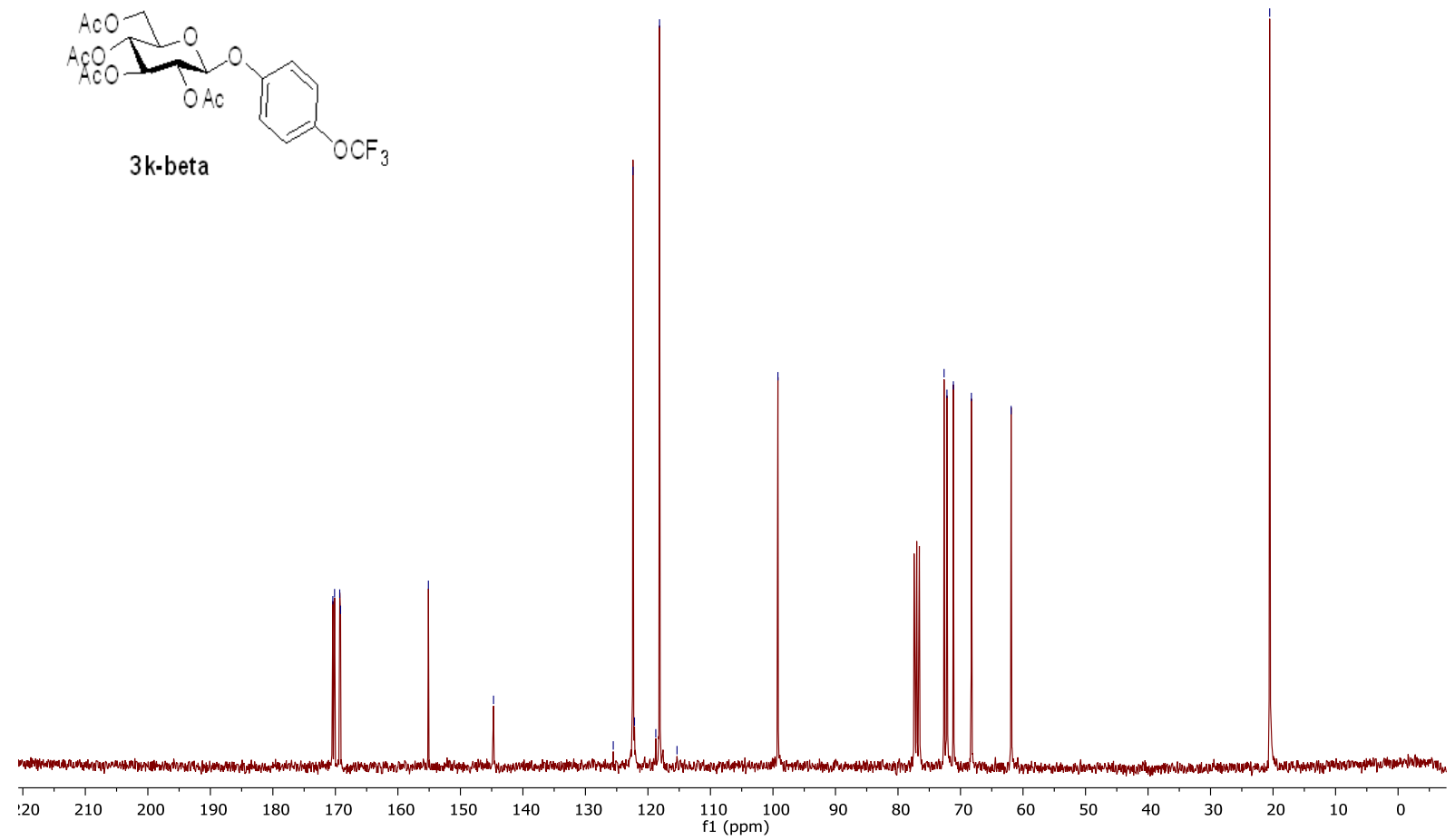


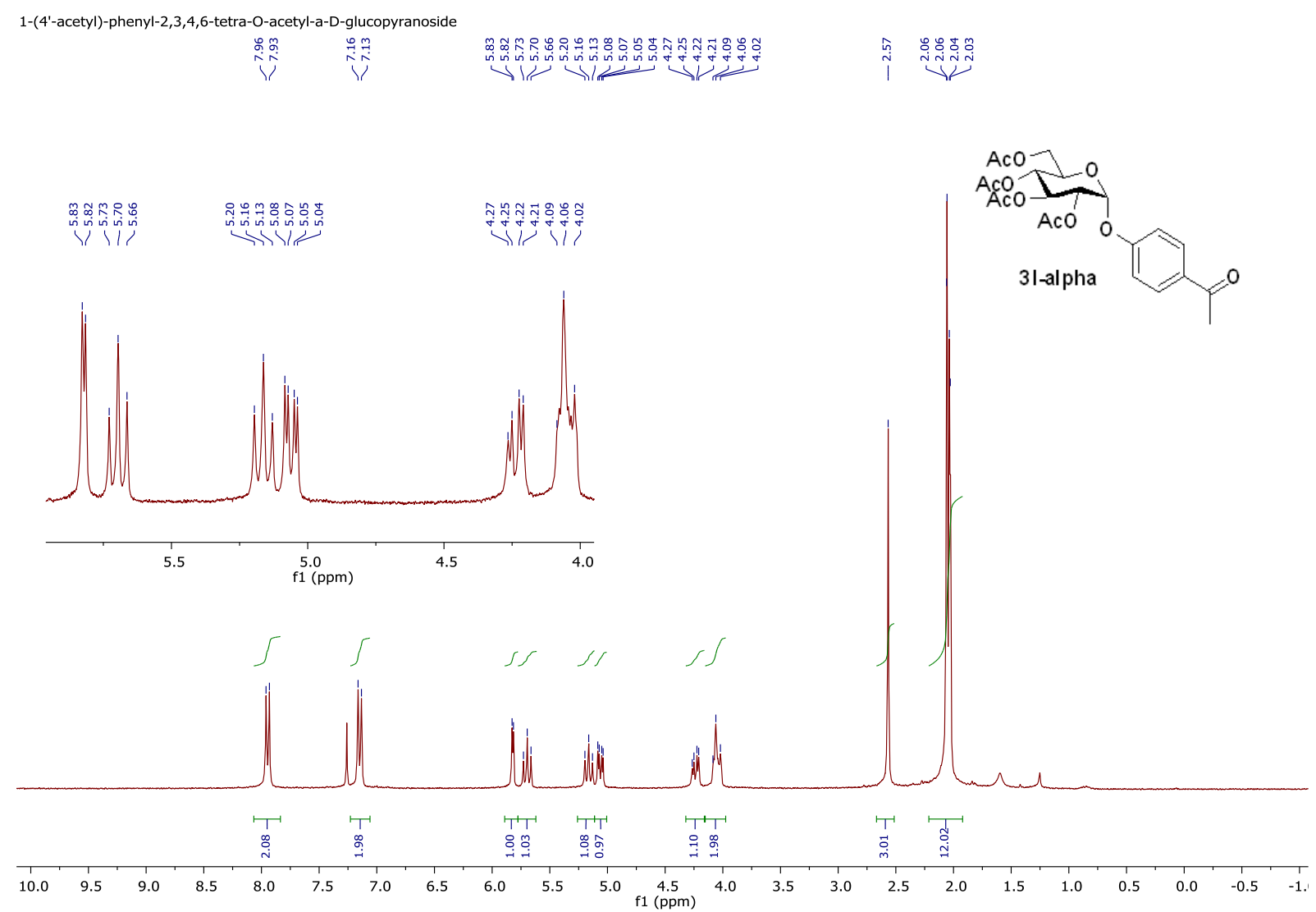

1-(4'-acetyl)-phenyl-2,3,4,6-tetra-O-acetyl-a-D-glucopyranoside

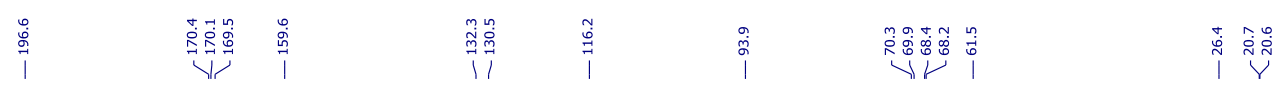

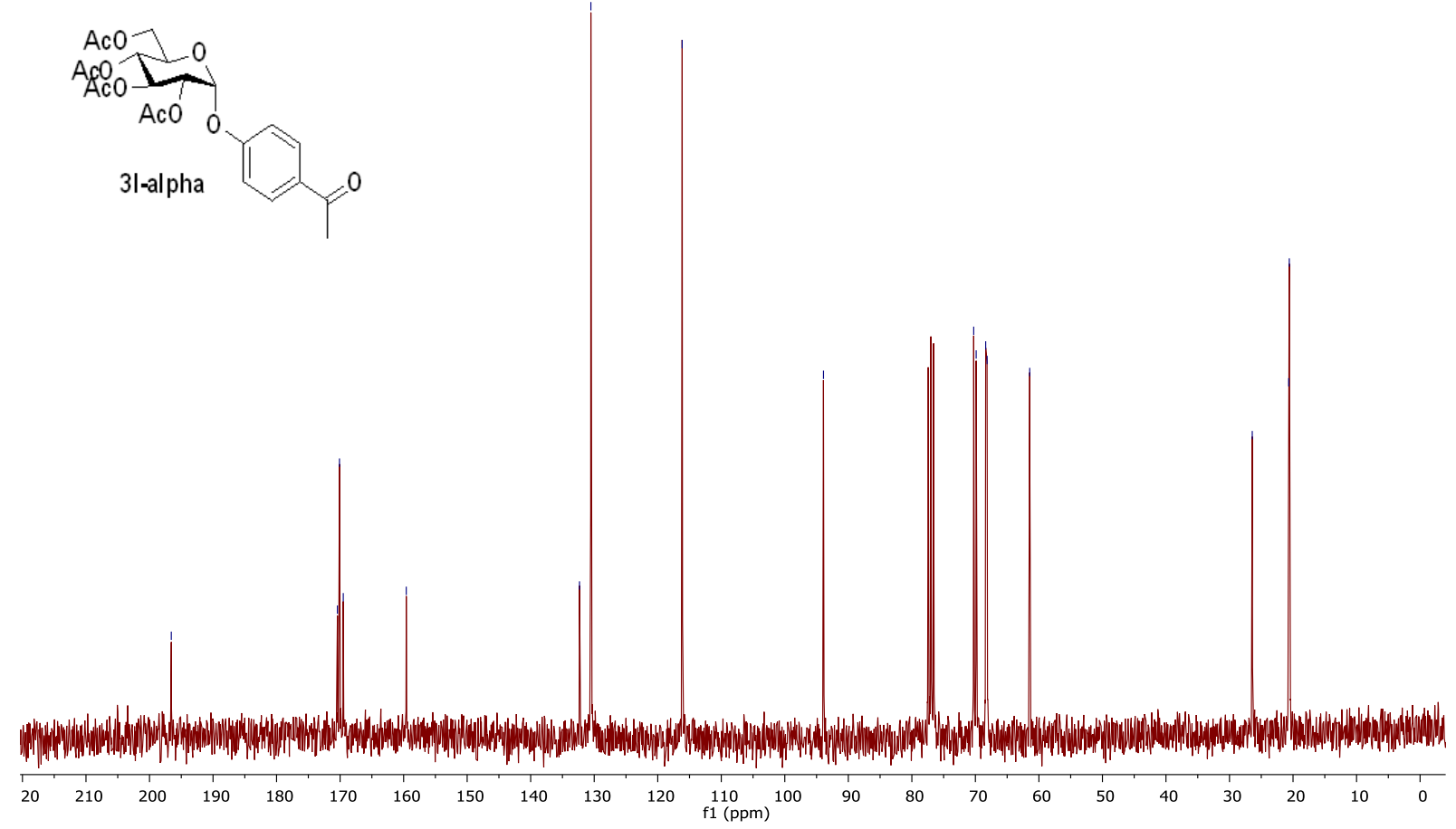


1-(4'-acetyl)-phenyl-2,3,4,6-tetra-O-acetyl-b-D-glucopyranoside

$$
\text { 至 }
$$
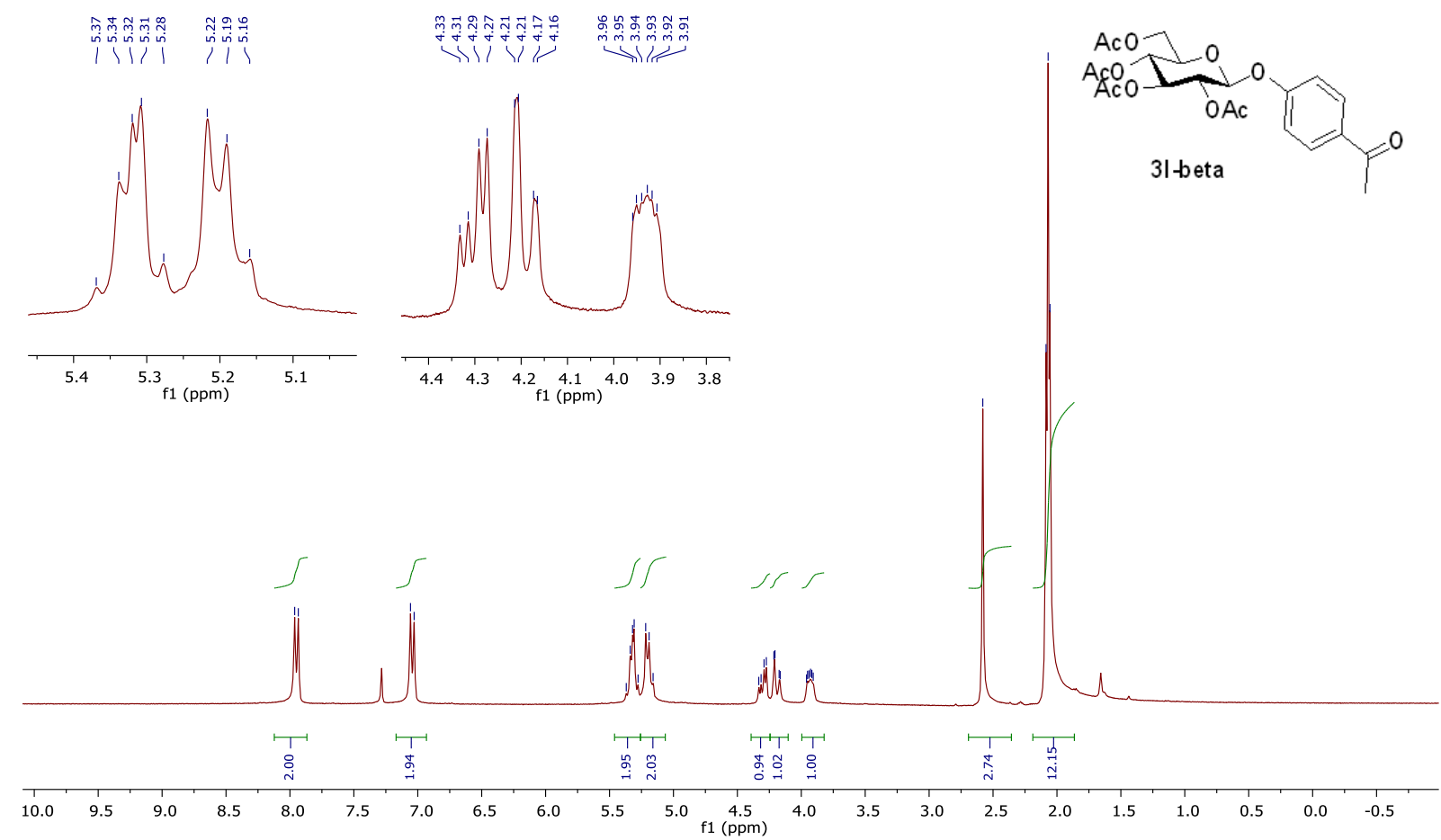

1-(4'-acetyl)-phenyl-2,3,4,6-tetra-O-acetyl-b-D-glucopyranoside

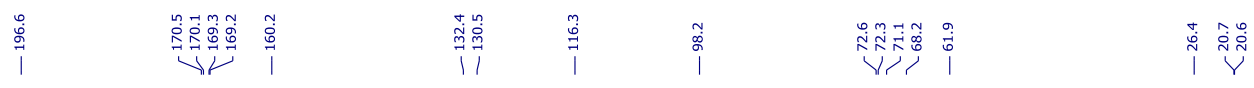

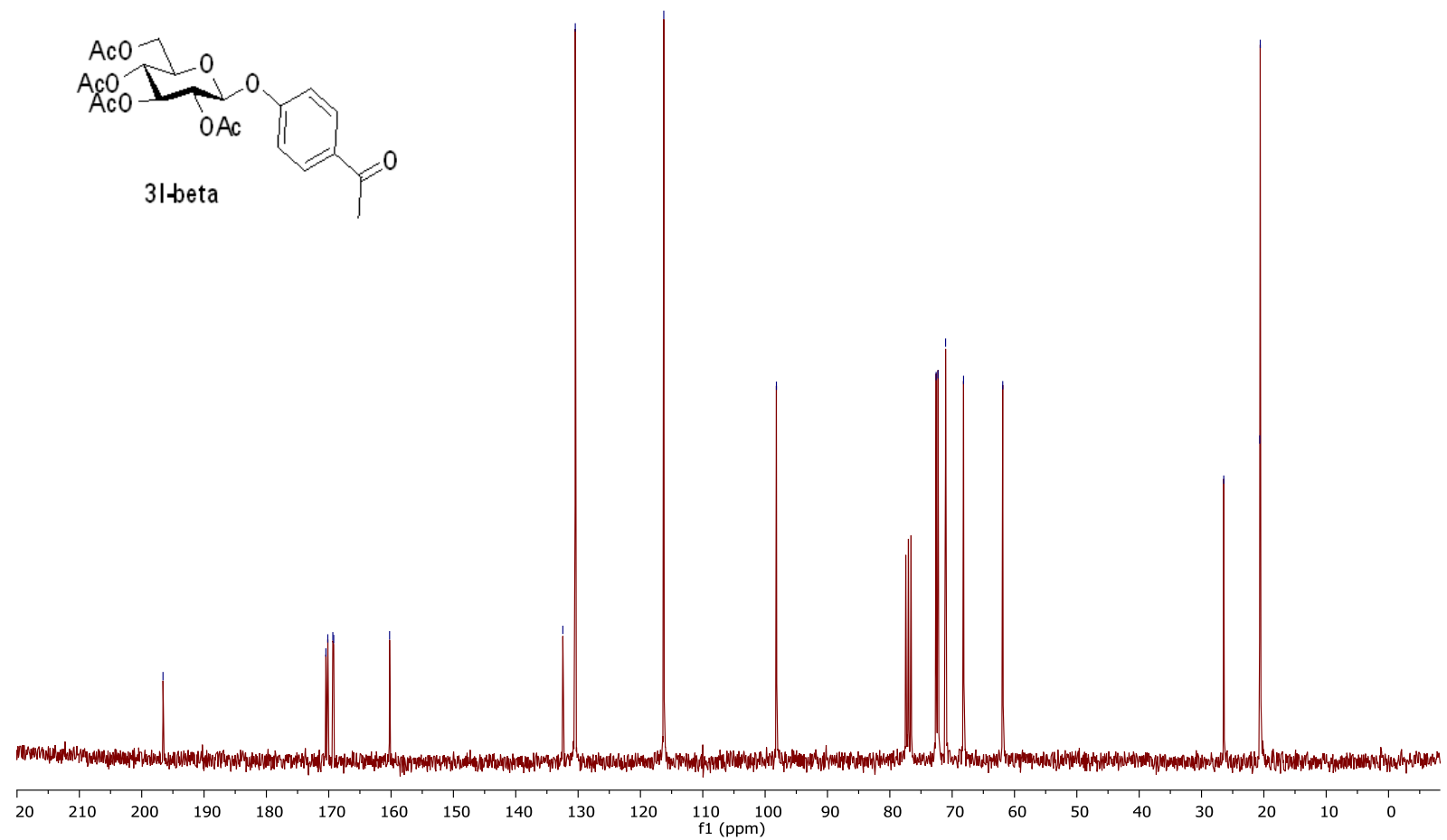




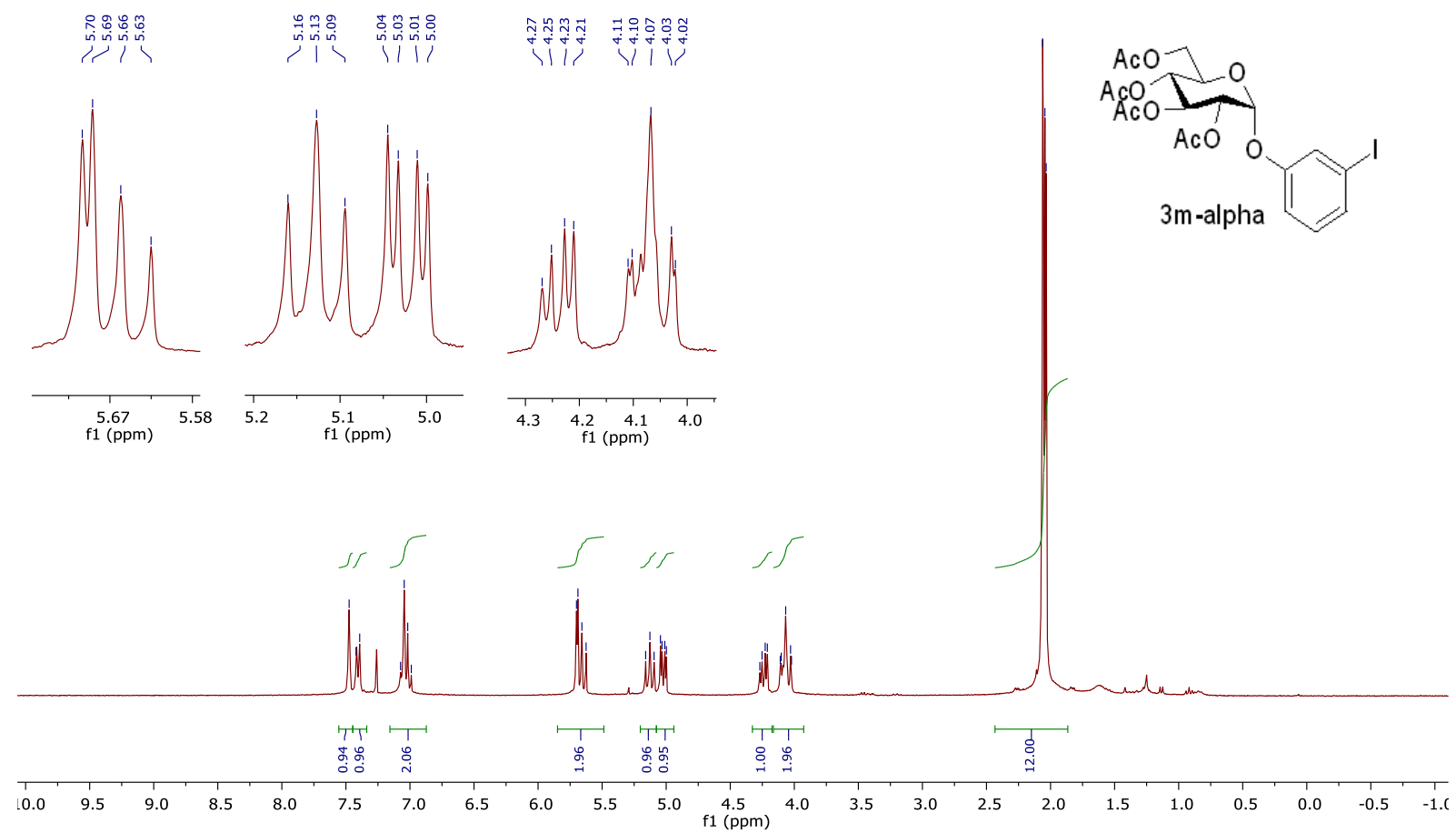

1-(3'-Iodo)-phenyl-2,3,4,6-tetra-O-acetyl-a-D-glucopyranoside

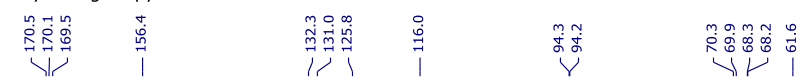
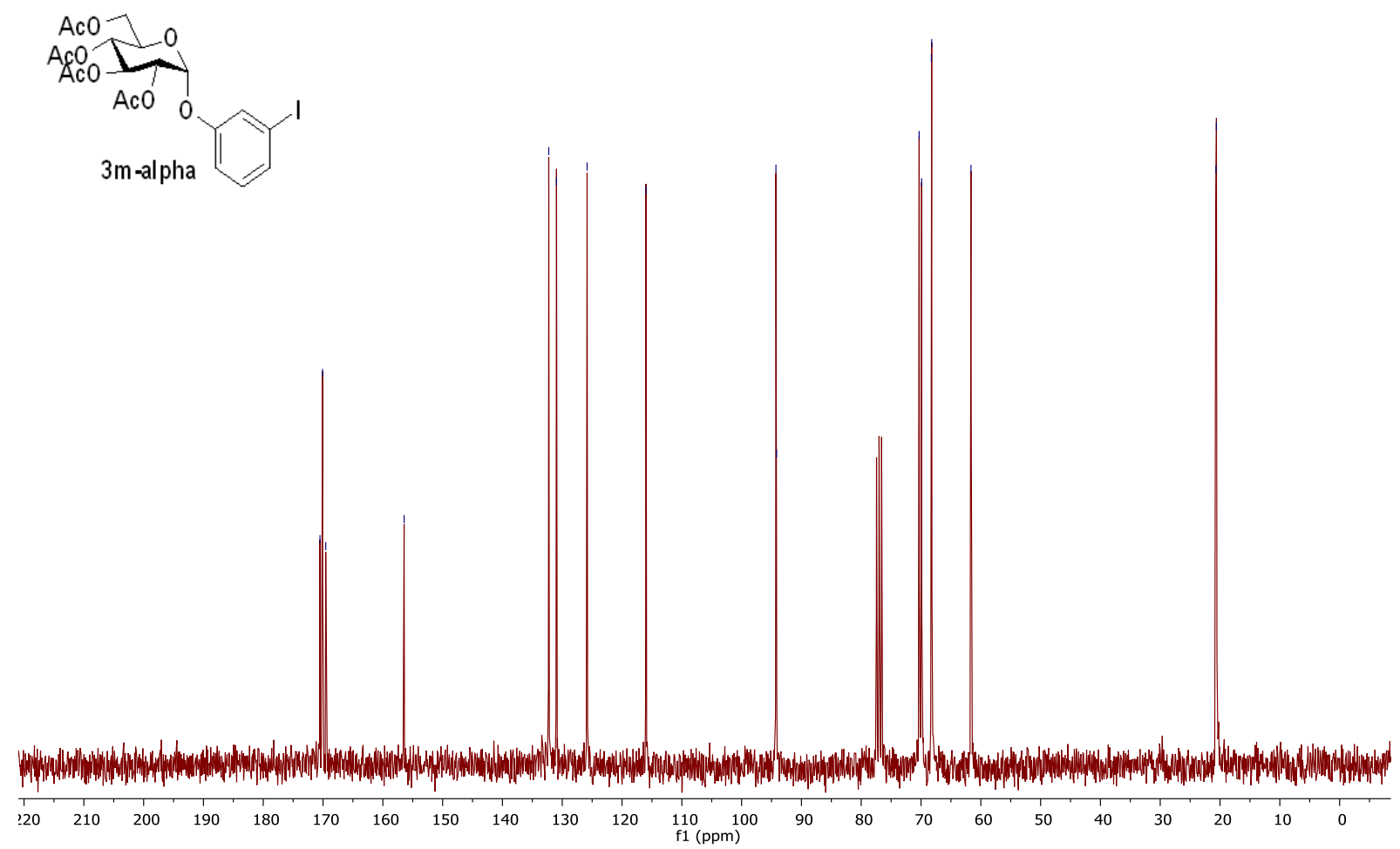
1-(3'-Iodo)-2,3,4,6-tetra-O-acetyl-b-D-glucopyranoside

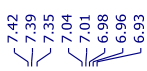

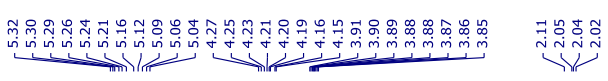

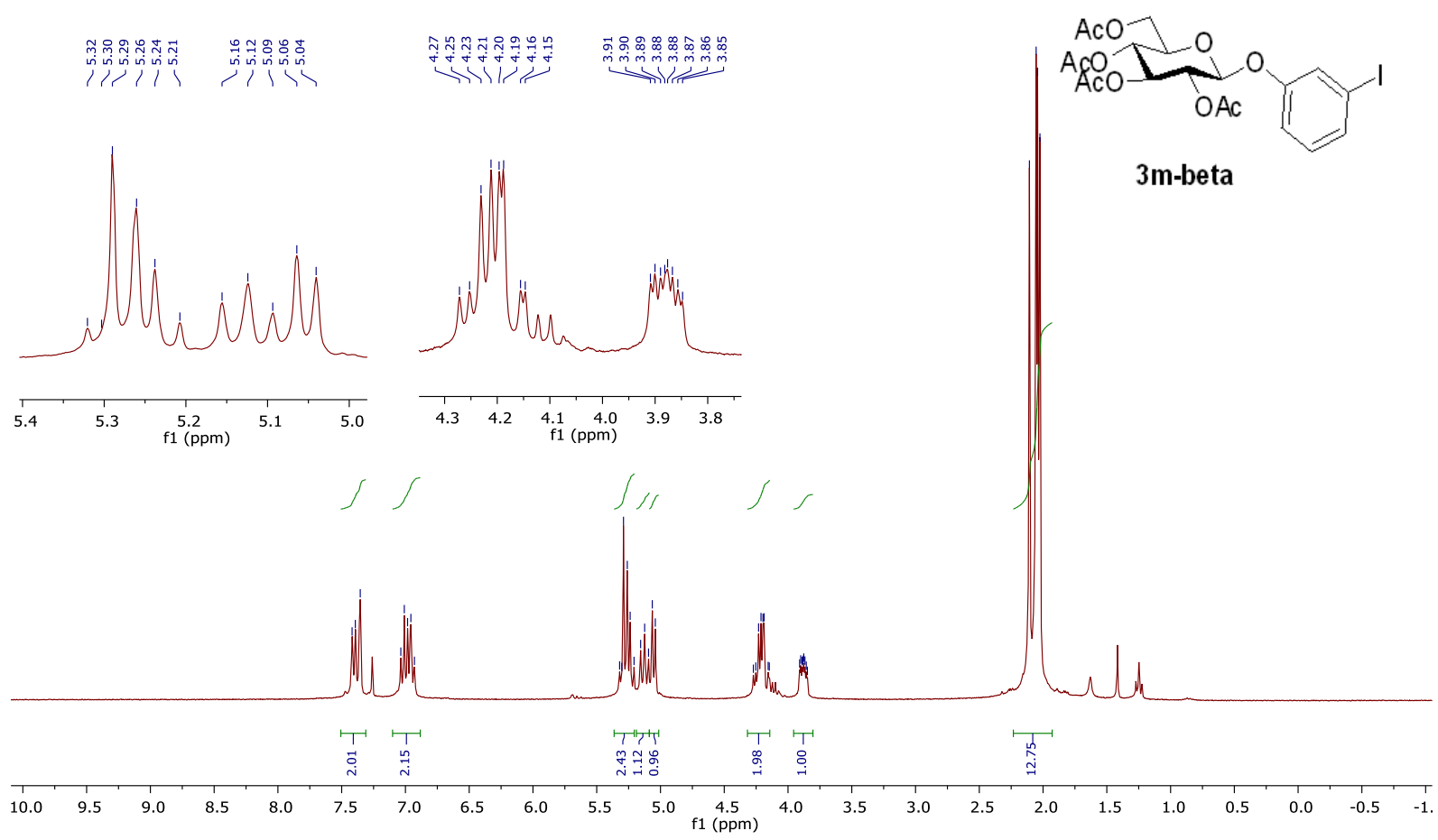

1-(3'-Iodo)-2,3,4,6-tetra-O-acetyl-b-D-glucopyranoside

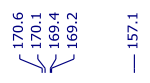

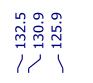

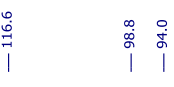

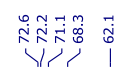

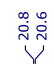

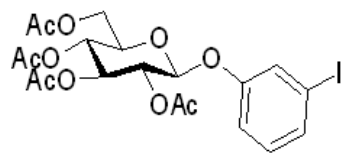

$3 \mathrm{~m}$-beta

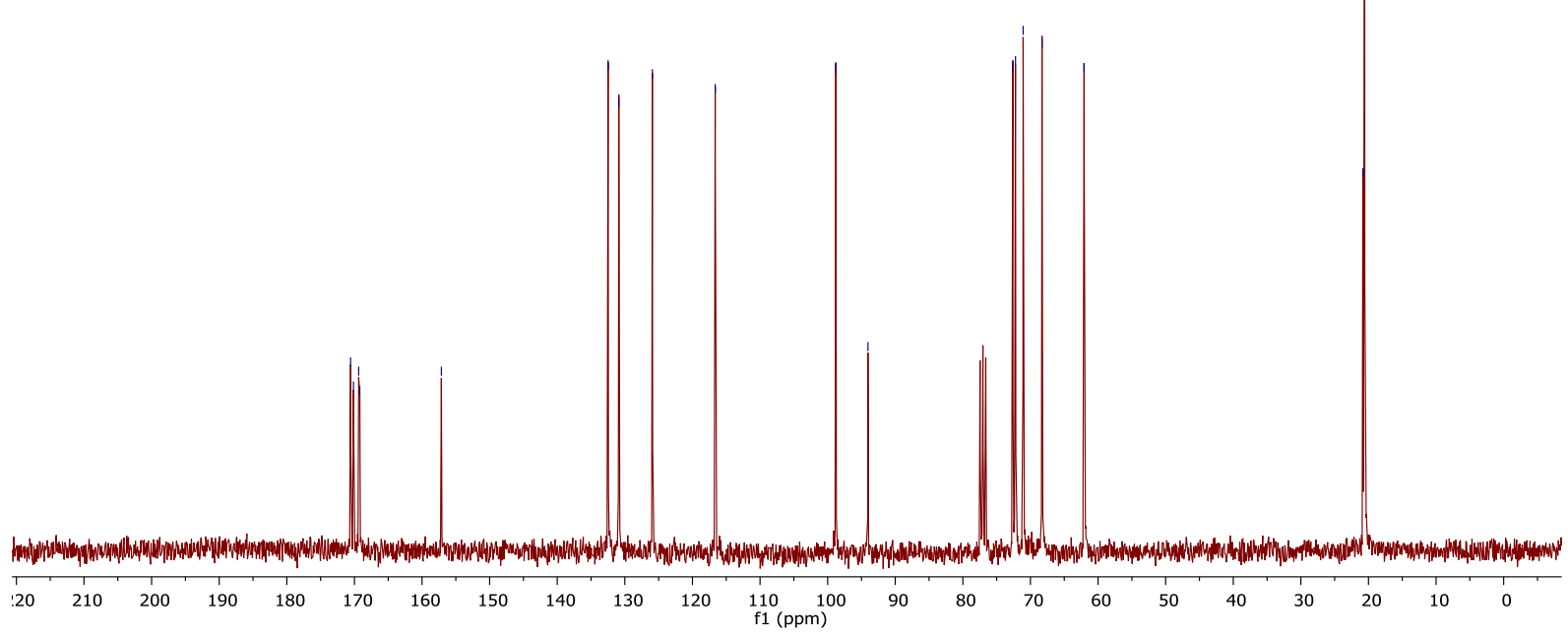

S27 


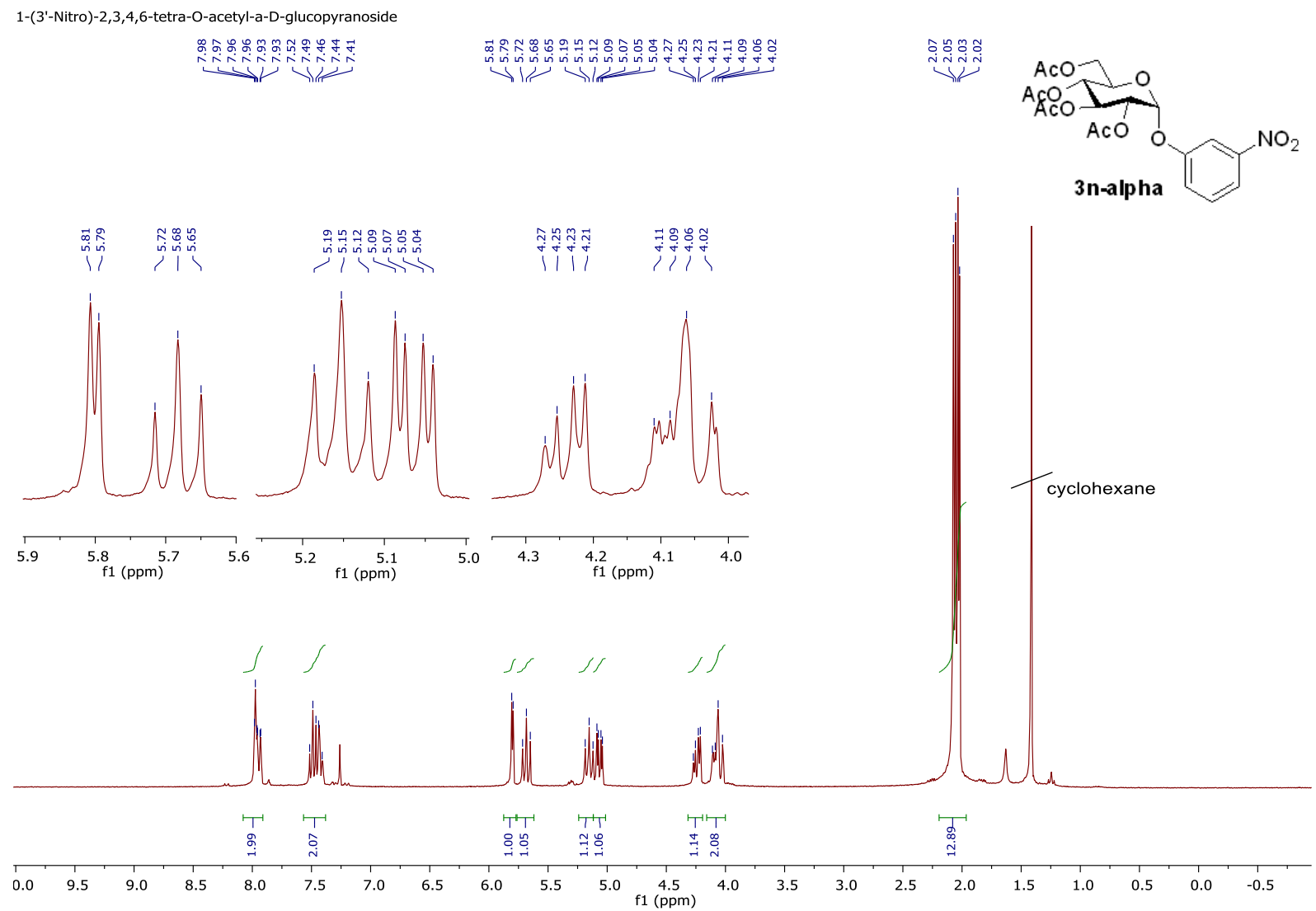

1-(3'-Nitro)-phenyl-2,3,4,6-tetra-O-acetyl-a-D-glucopyranoside

$$
\text { 至 }
$$
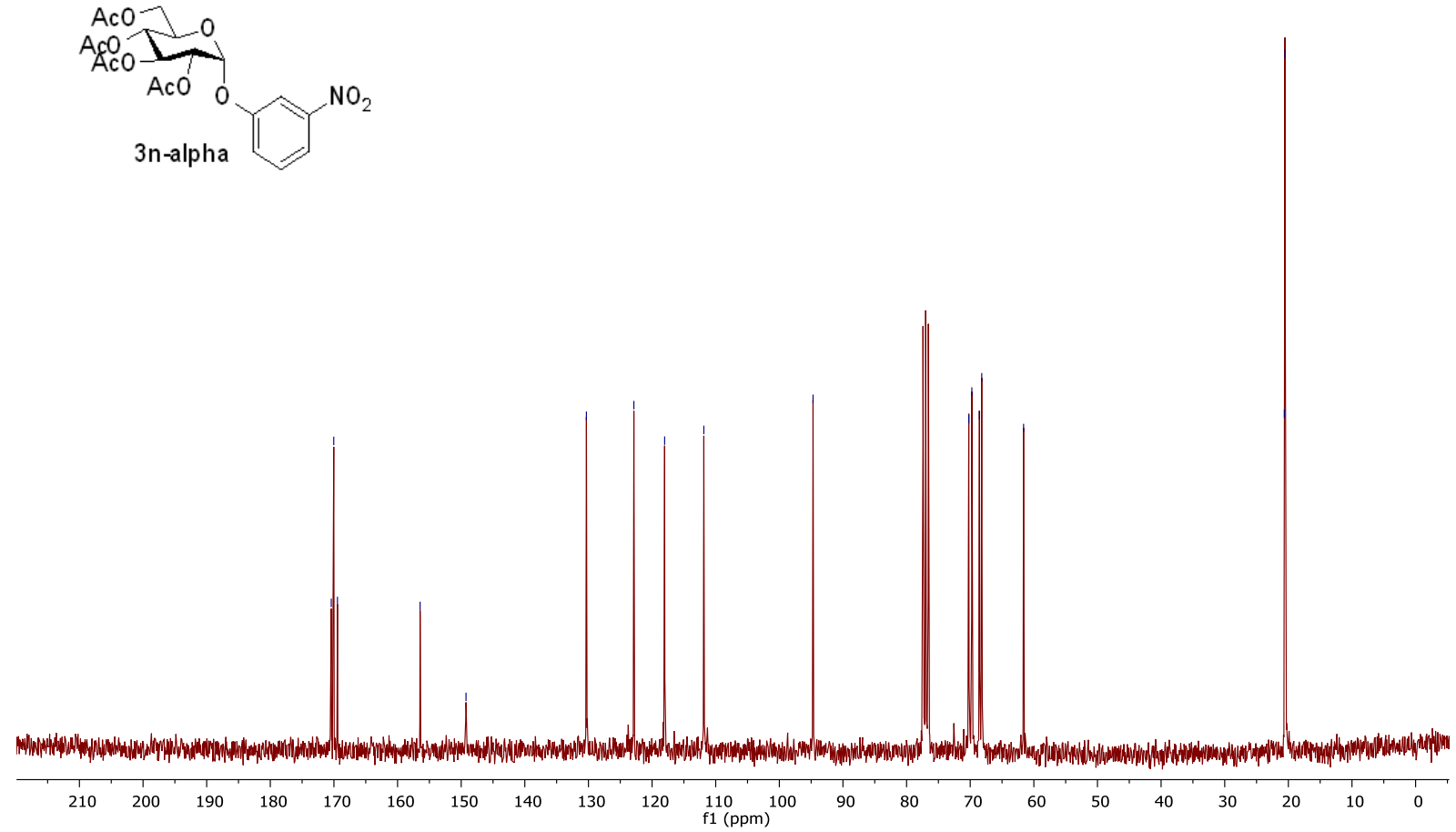

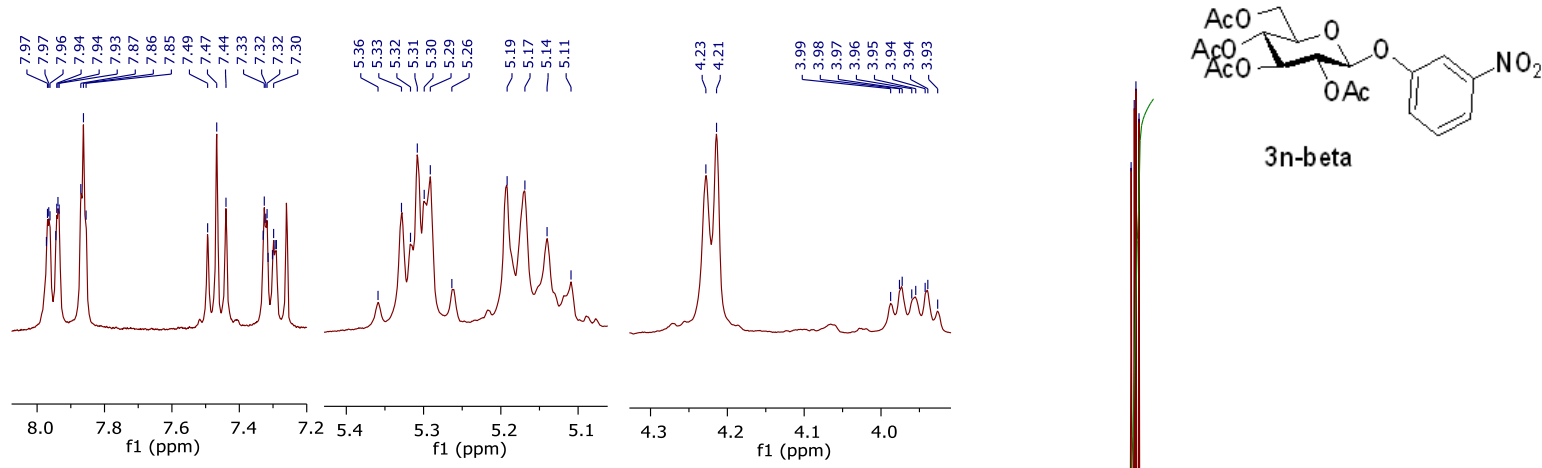

$3 n-b e t a$

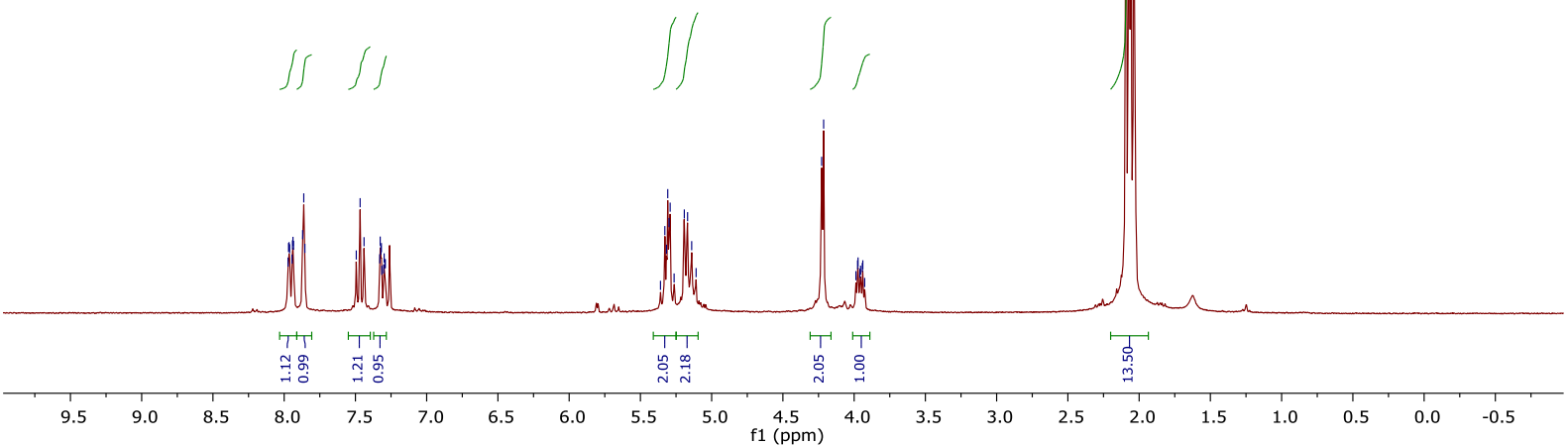

1-(3'-Nitro)-2,3,4,6-tetra-O-acetyl-b-D-glucopyranoside

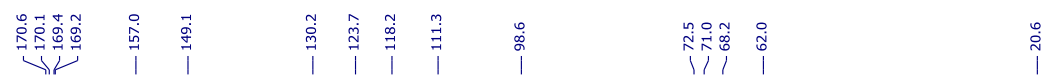

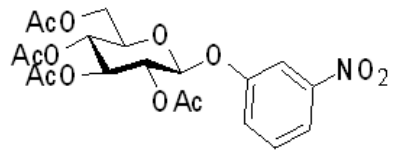

$3 n-b$ eta

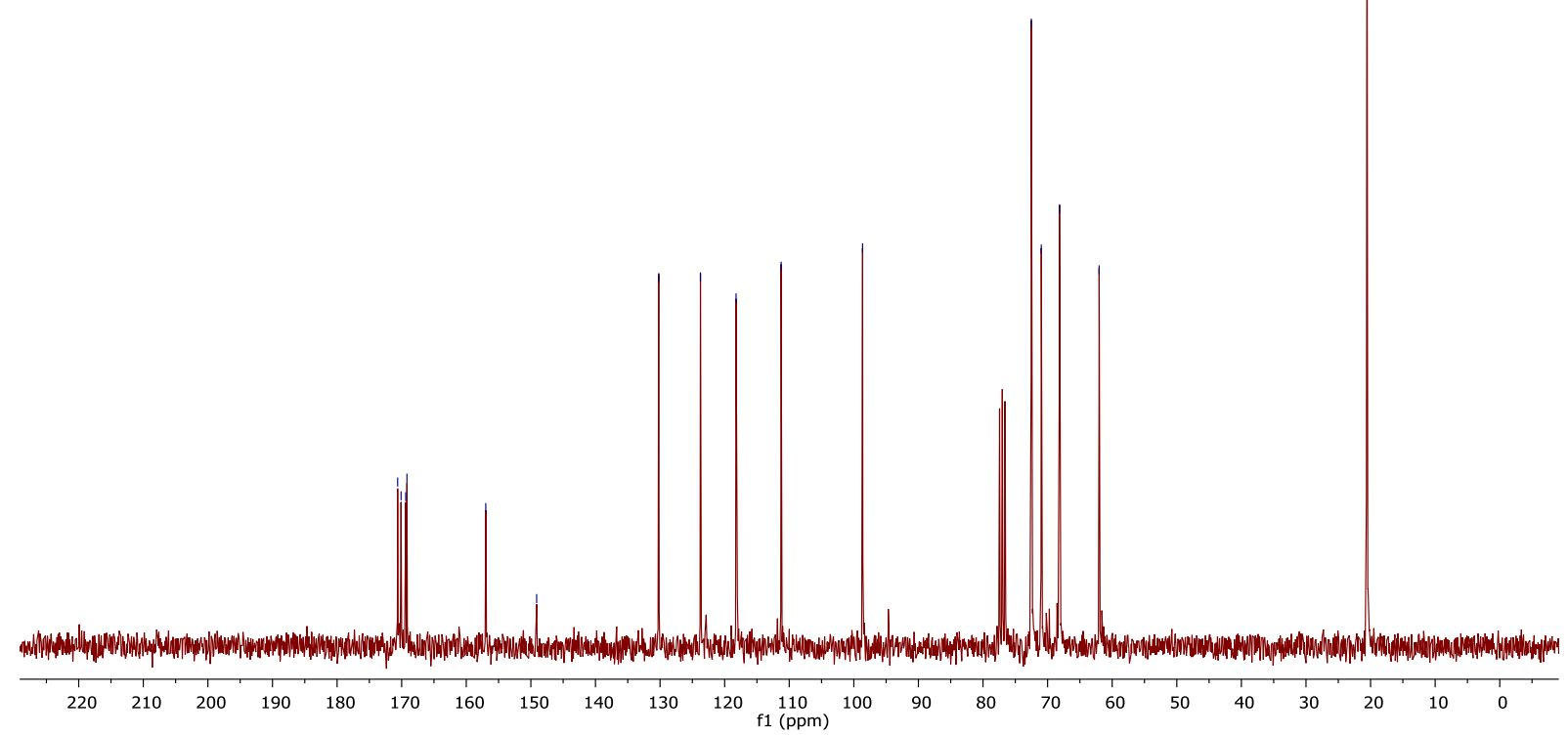




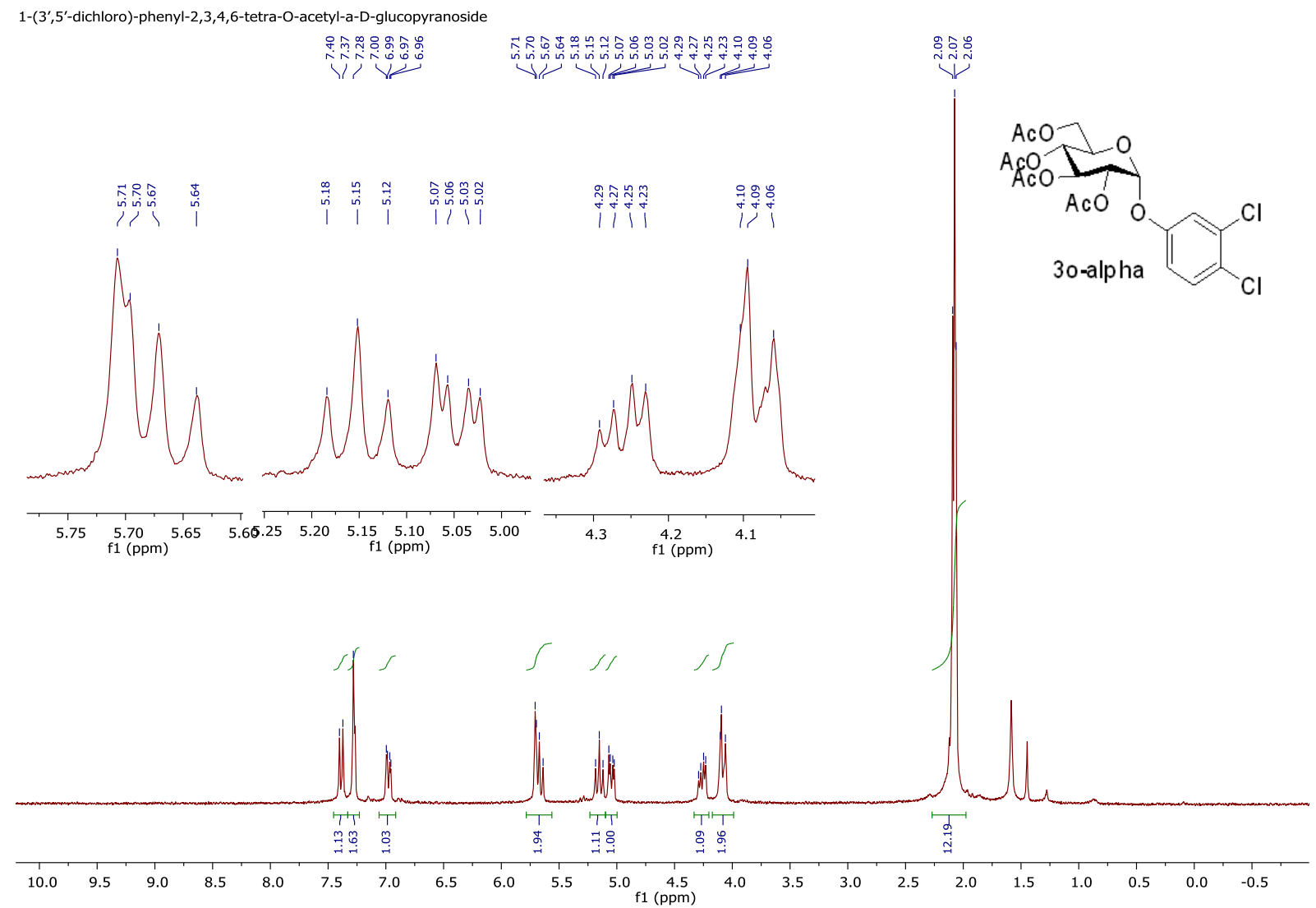

1-(3',5'-dichloro)-phenyl-2,3,4,6-tetra-O-acetyl-a-D-glucopyranoside

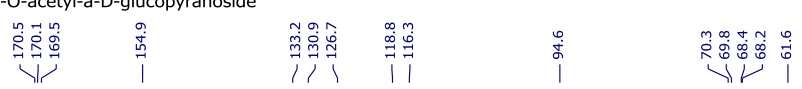
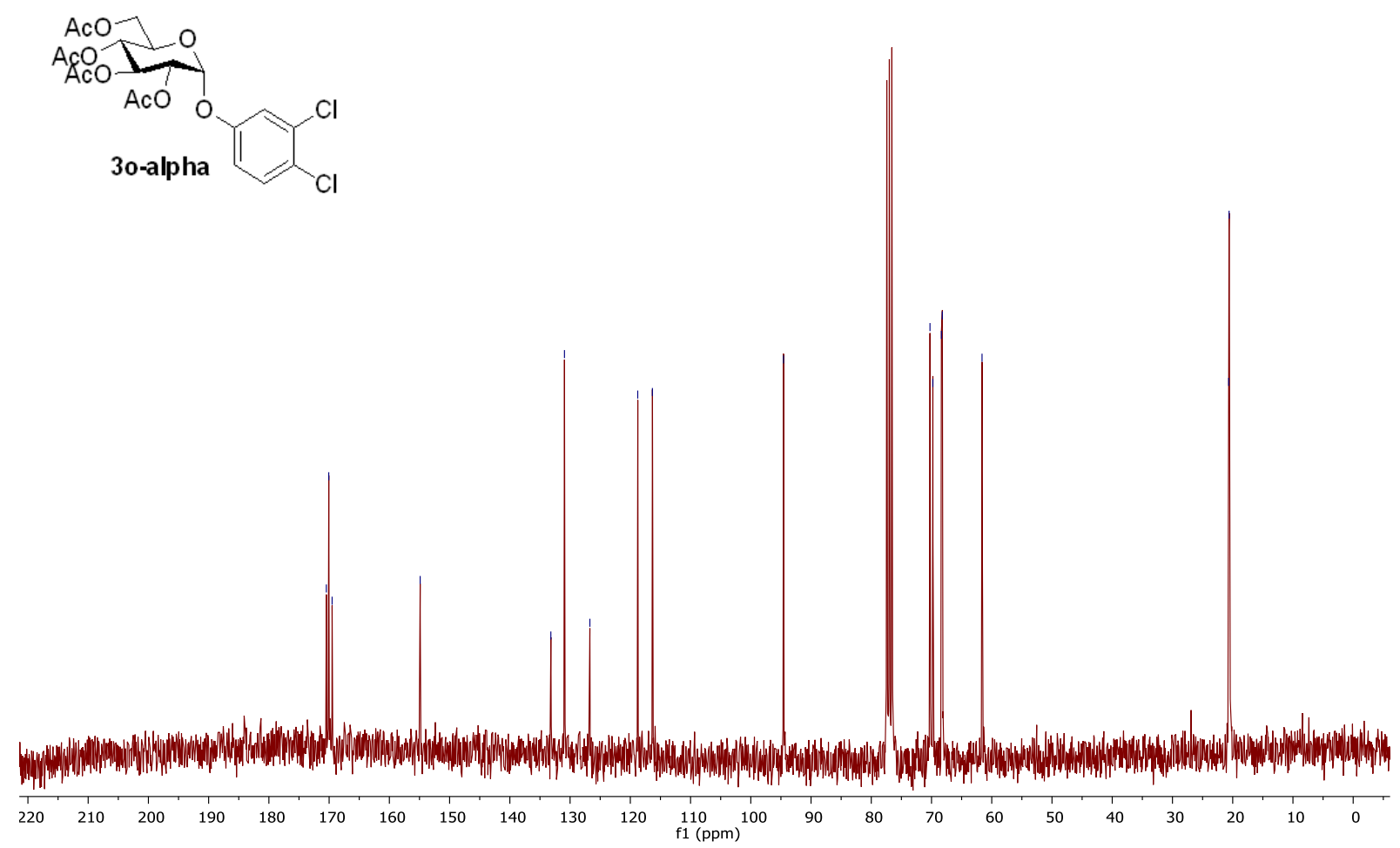


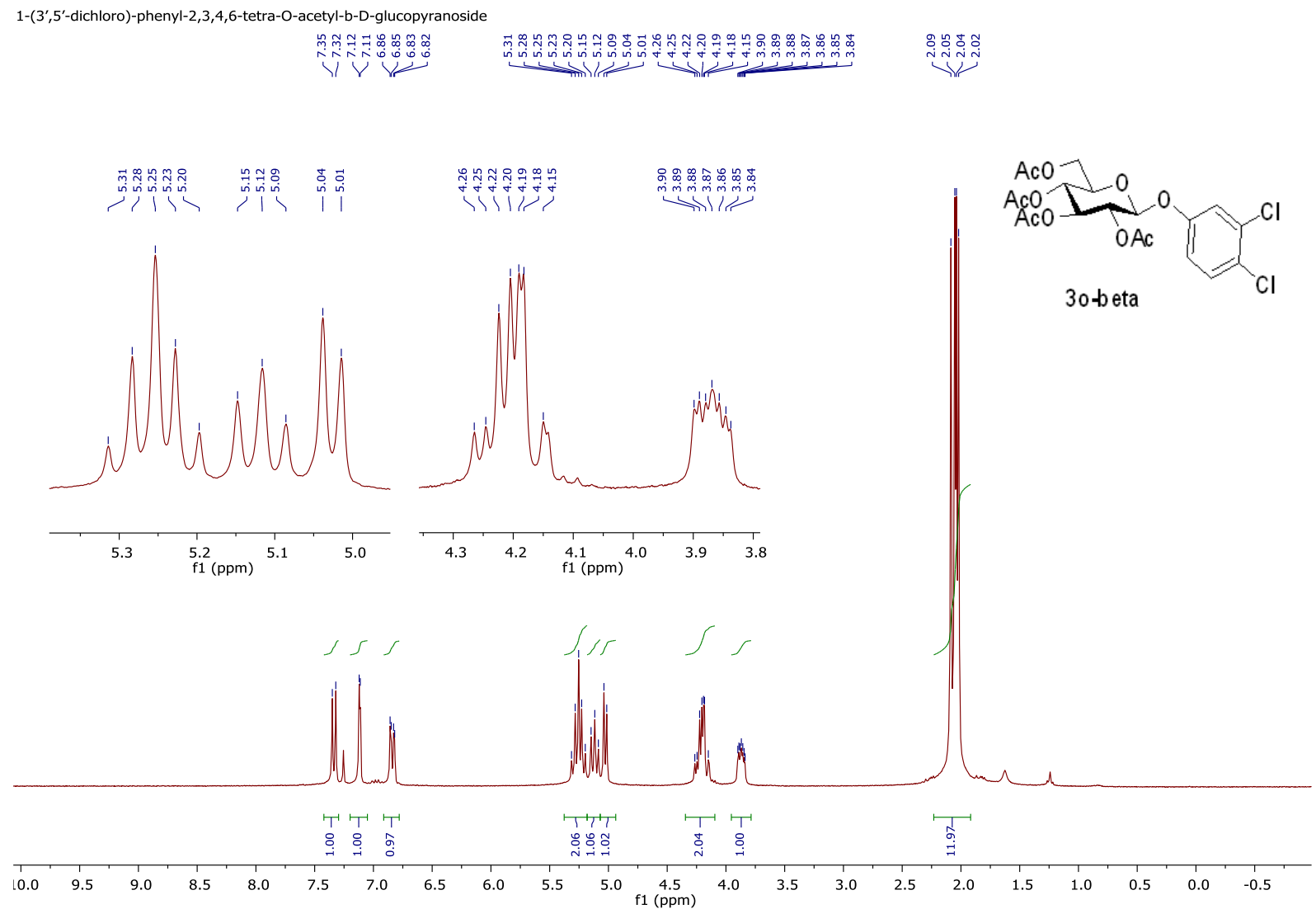

1-(3',5'-dichloro)-phenyl-2,3,4,6-tetra-O-acetyl-b-D-glucopyranoside

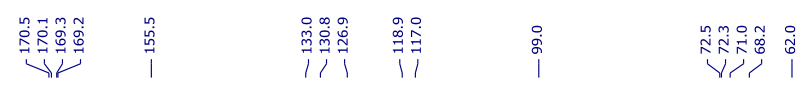

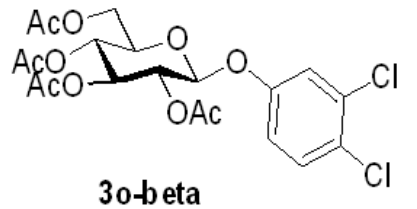

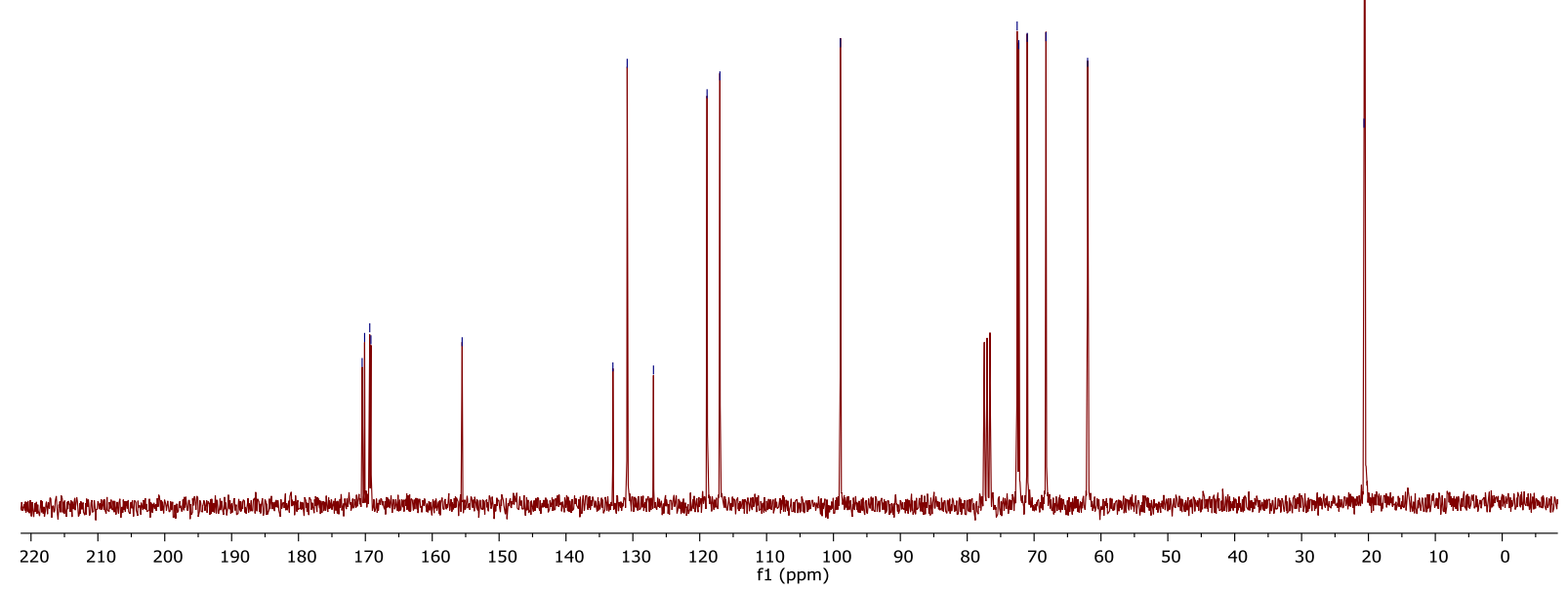




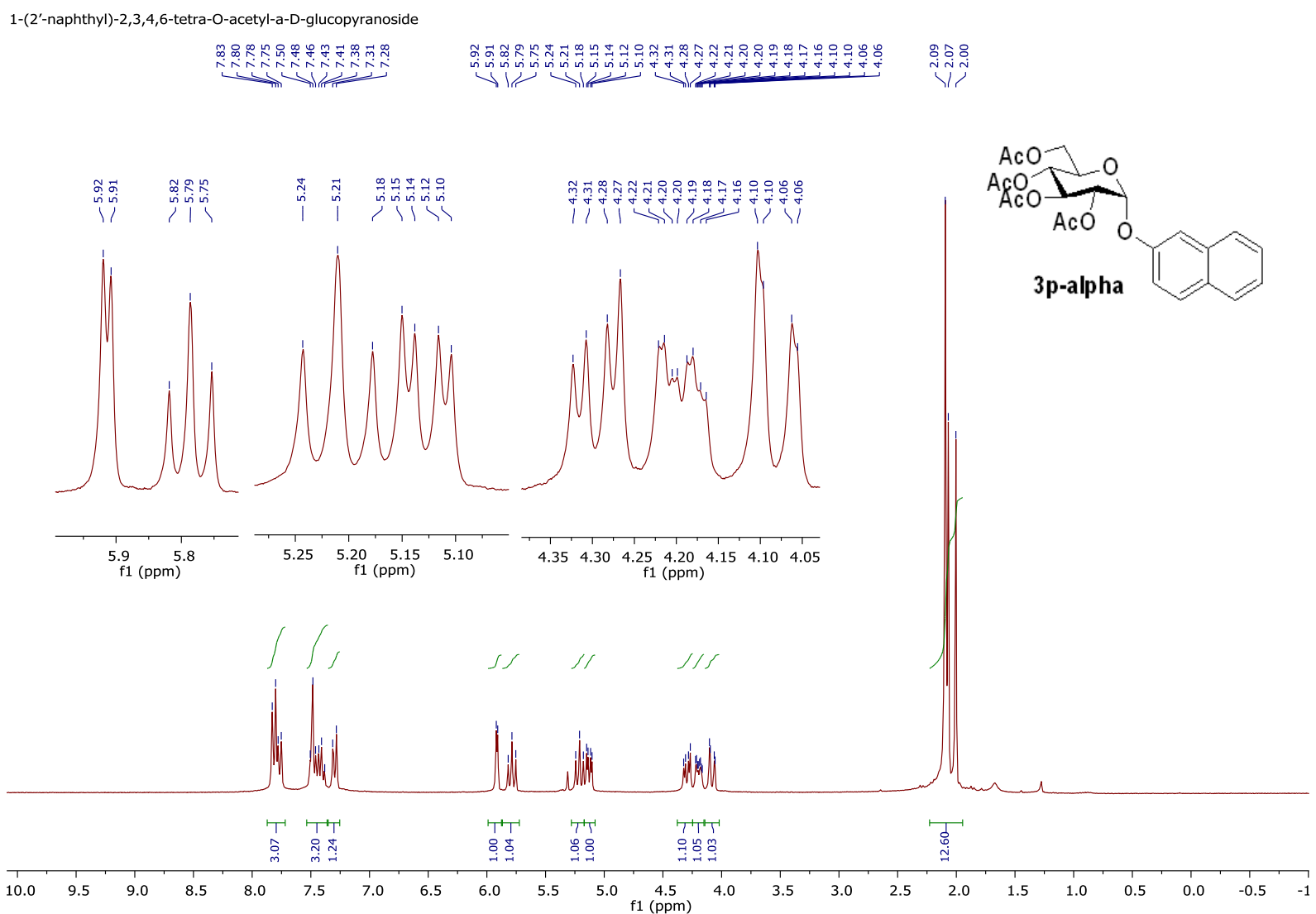

1-(2'-naphthyl)-2,3,4,6-tetra-O-acetyl-a-D-glucopyranoside

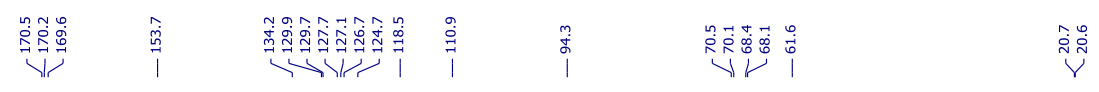
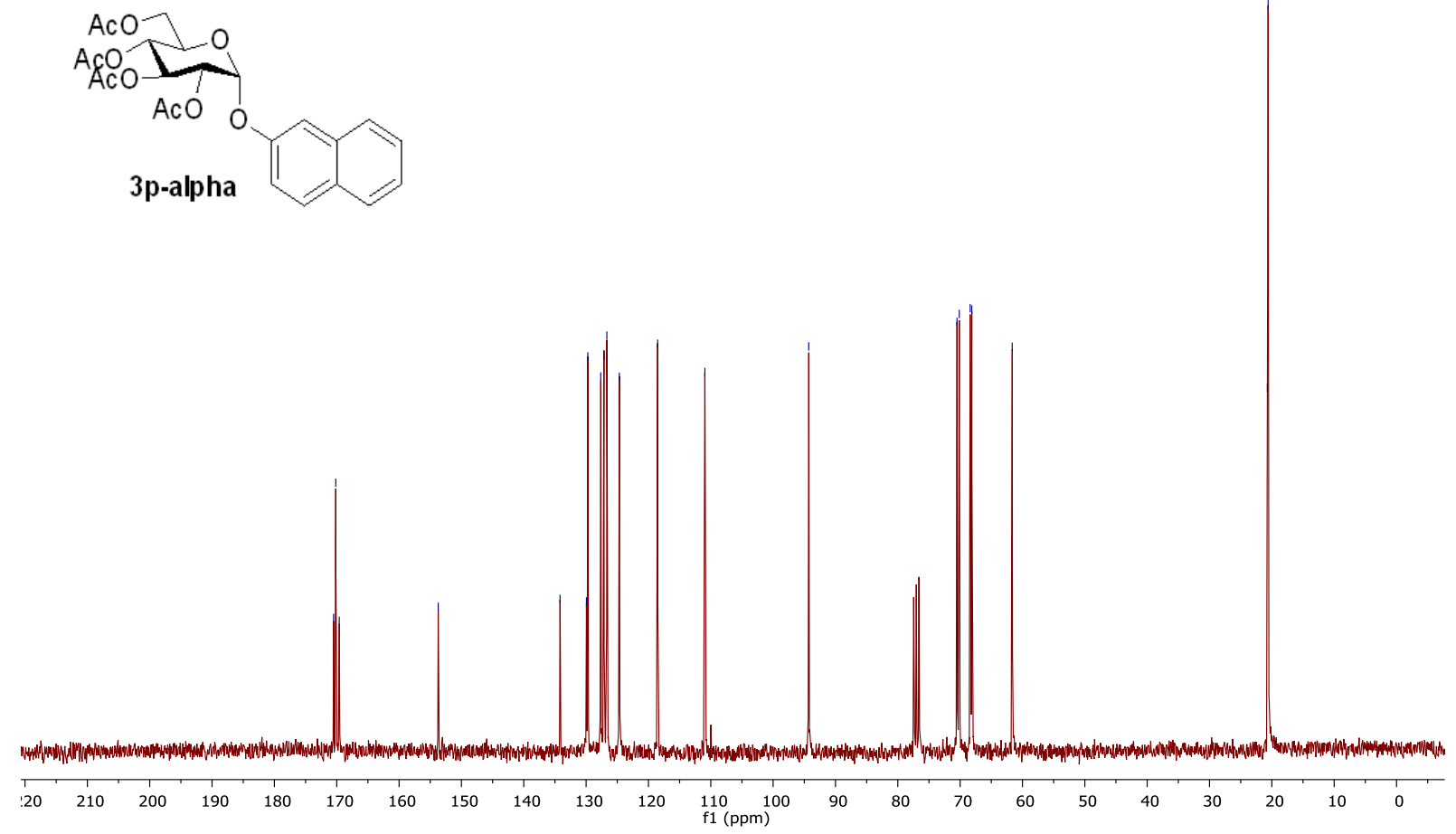
1-(2'-naphthyl)-2,3,4,6-tetra-O-acetyl-b-D-glucopyranoside

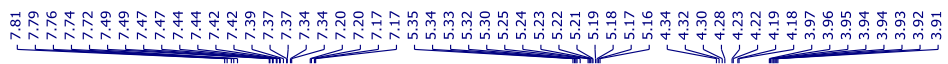

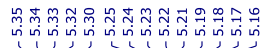

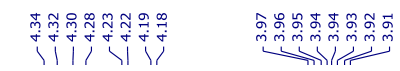
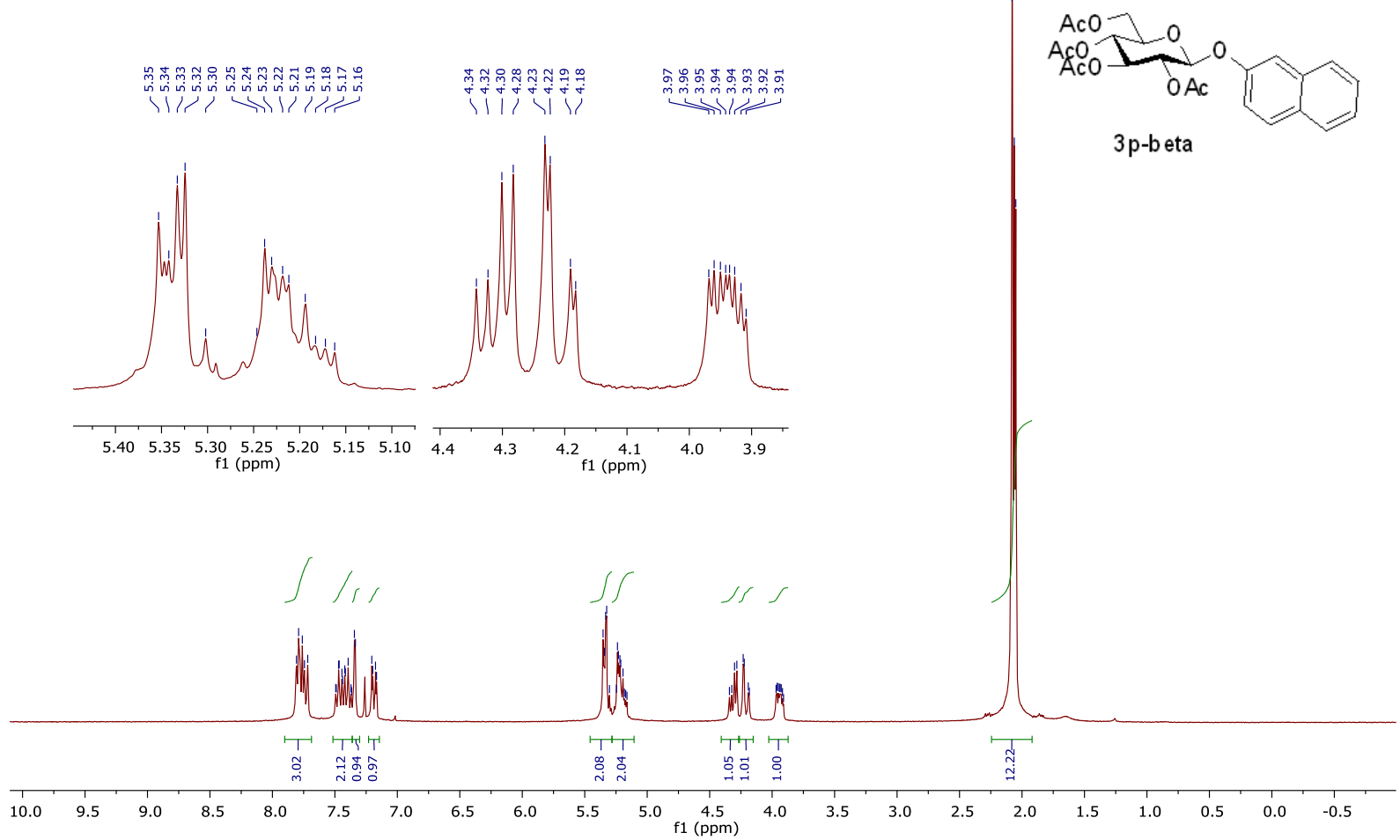

1-(2'-naphthyl)-2,3,4,6-tetra-O-acetyl-b-D-glucopyranoside

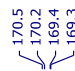

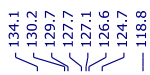

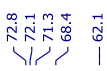

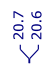
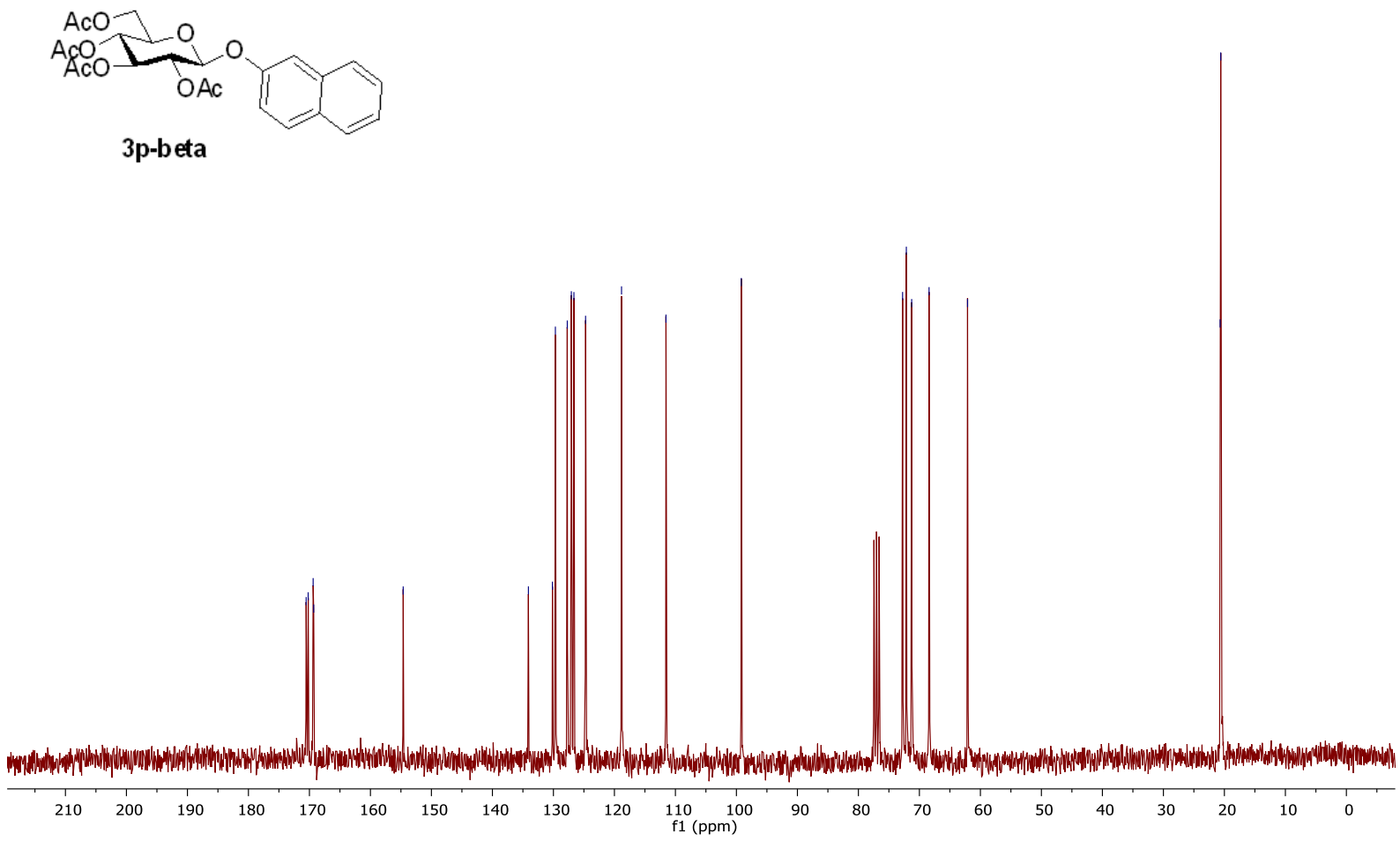

S33 


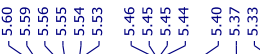

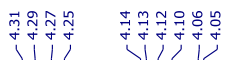
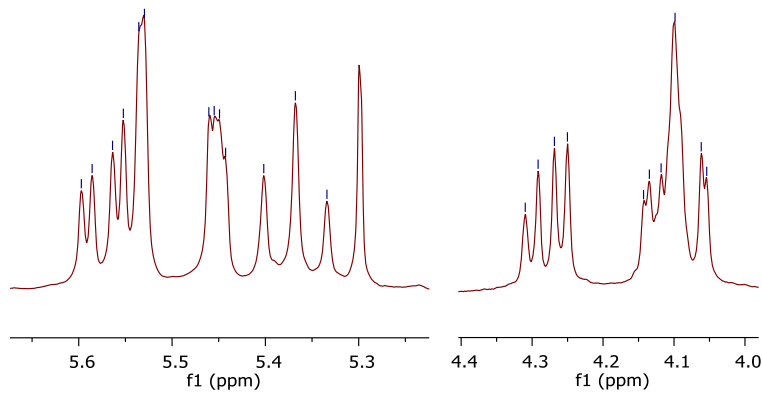

AcO

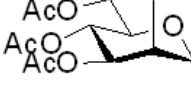

4b-alpha
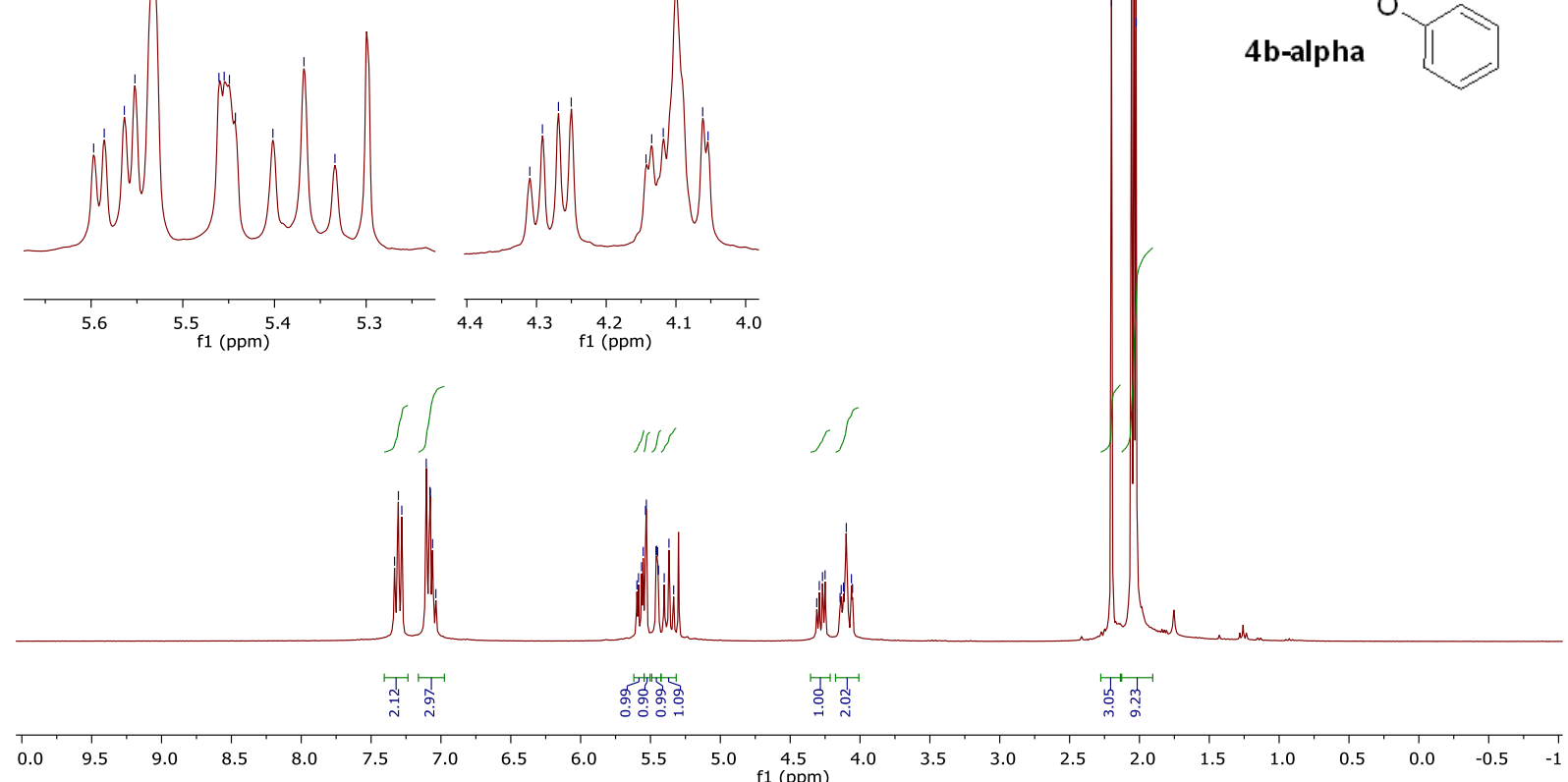

1-Phenyl-2,3,4,6-tetra-O-acetyl-a-D-mannopyranoside

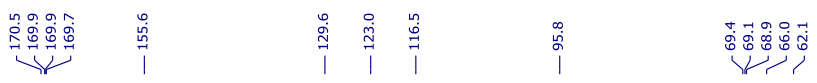

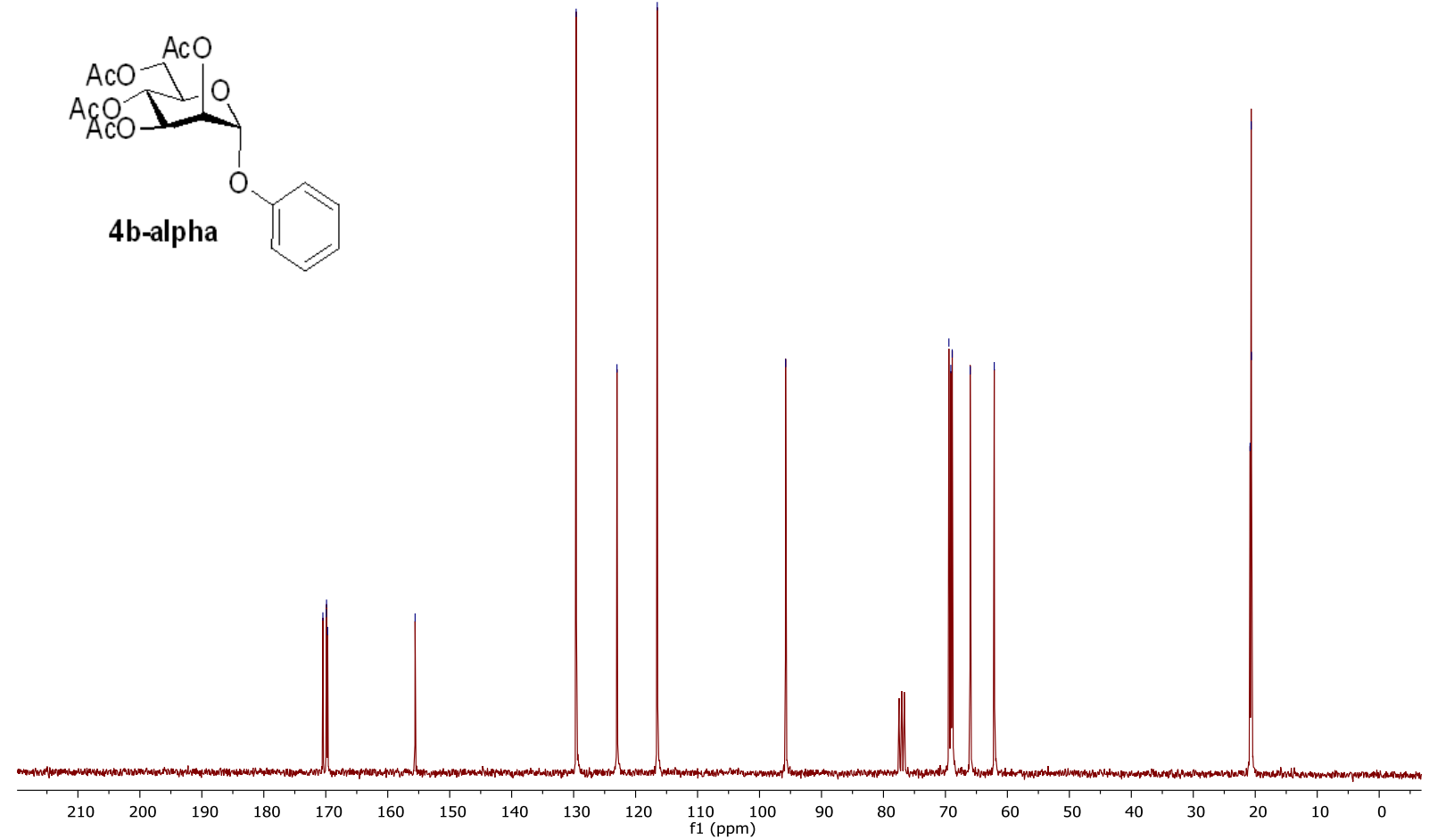


1-Phenyl-2,3,4,6-tetra-O-acetyl-b-D-mannopyranoside

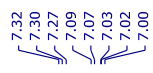

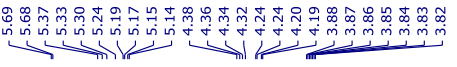

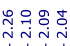

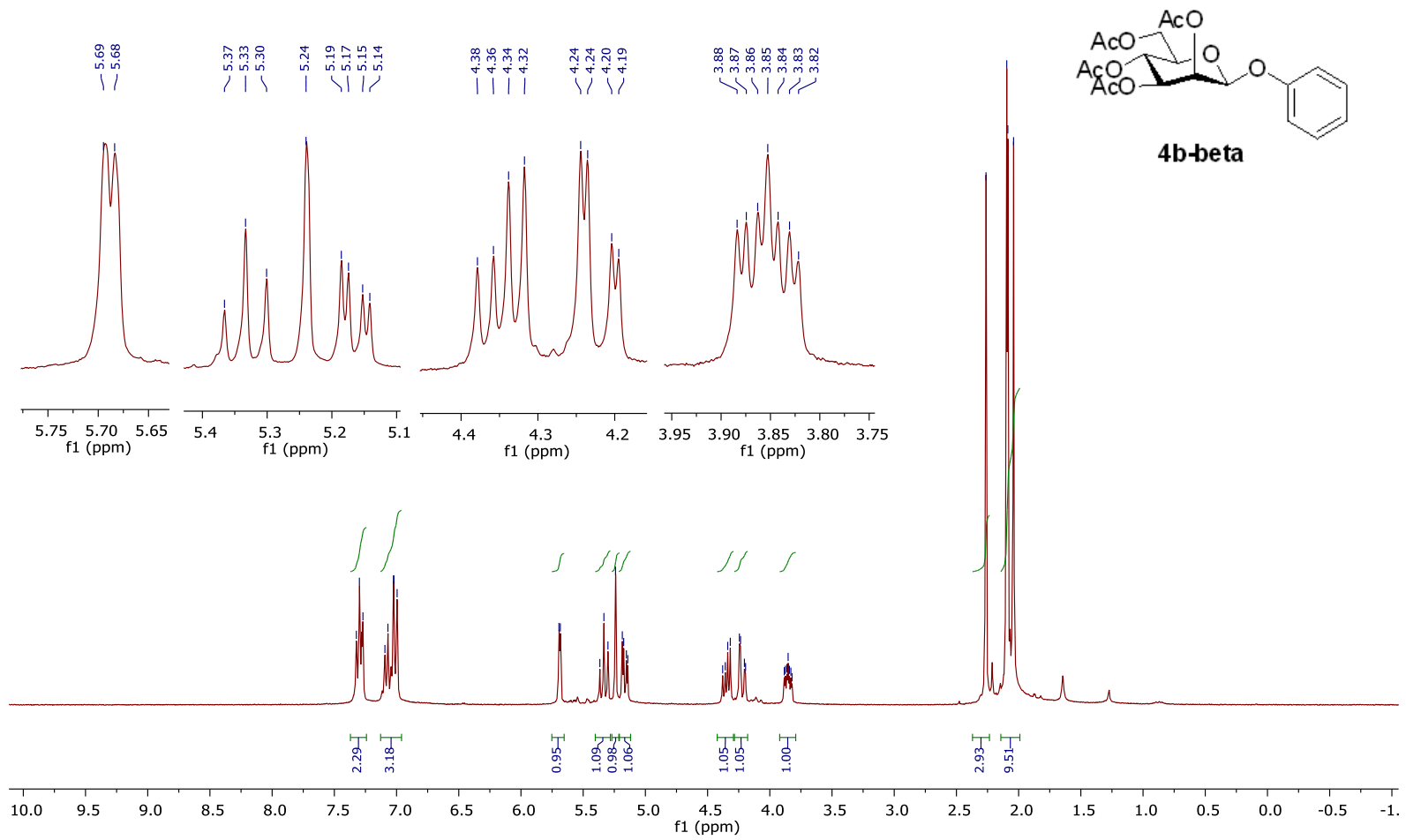

1-Phenyl-2,3,4,6-tetra-O-acetyl-b-D-mannopyranoside

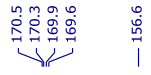

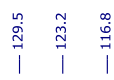

@òn

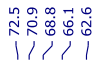

ขึํำ

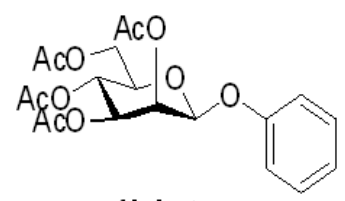

4b-beta

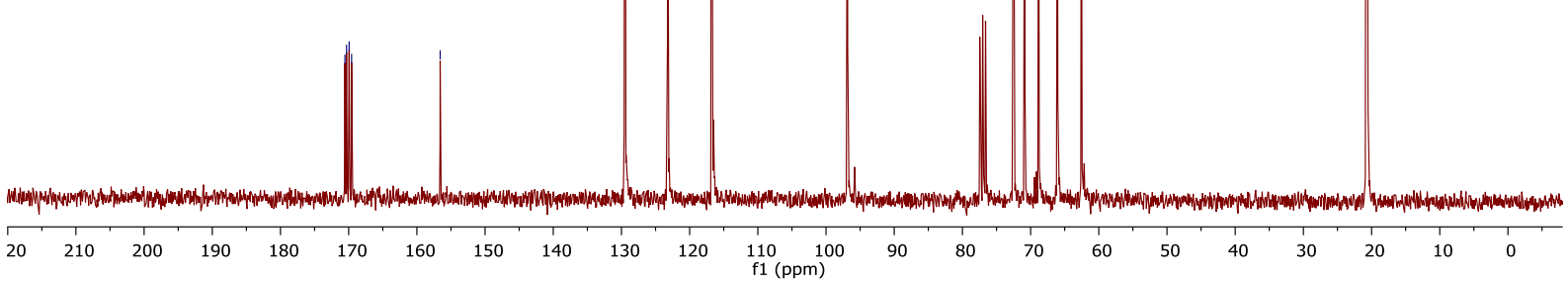

S35 


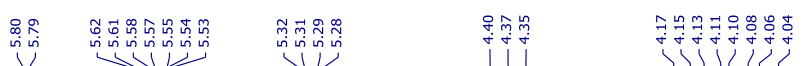

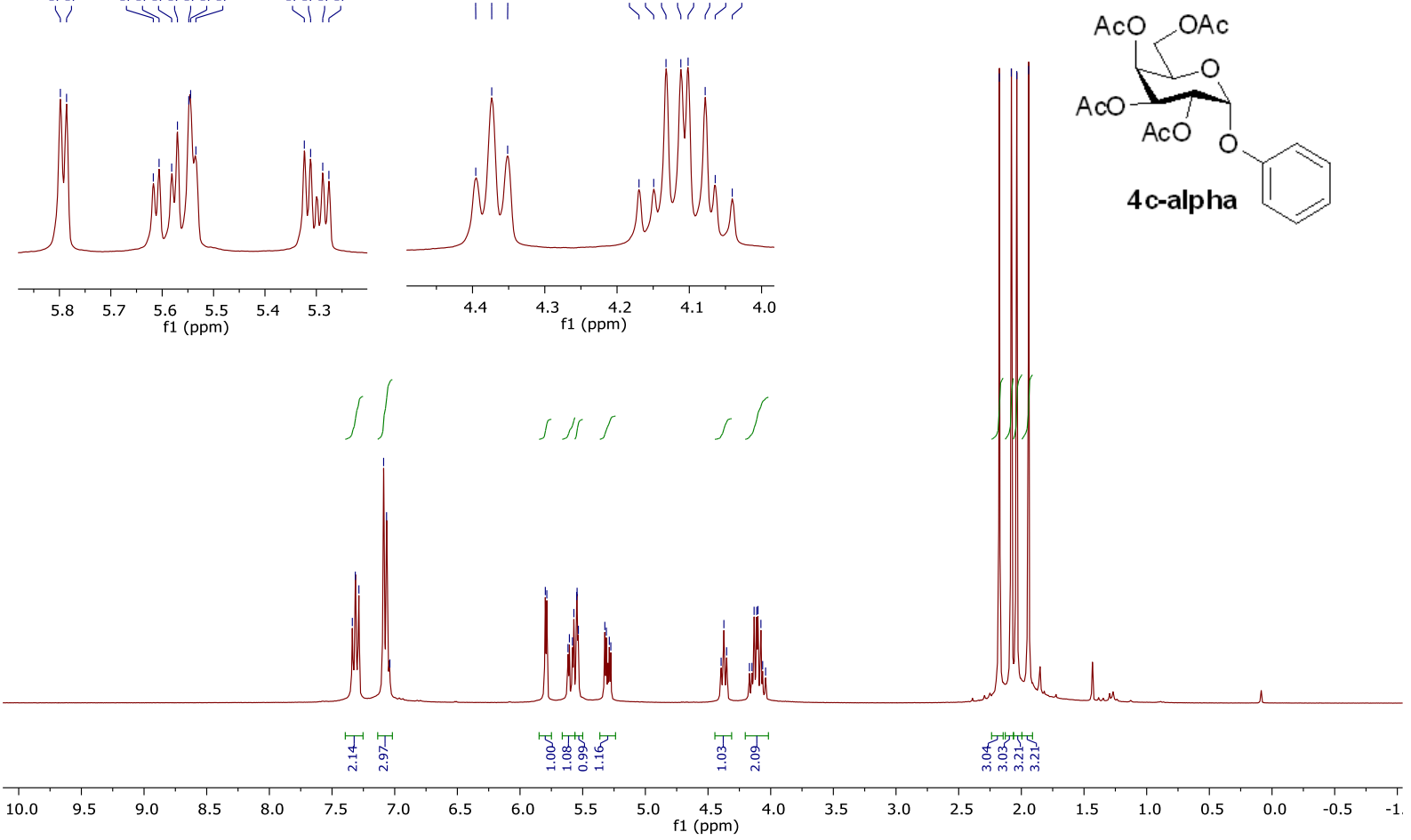

1-Phenyl-2,3,4,6-tetra-O-acetyl-a-D-galactopyranoside

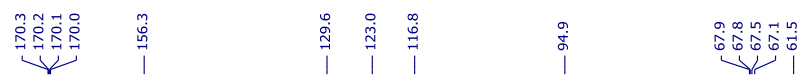
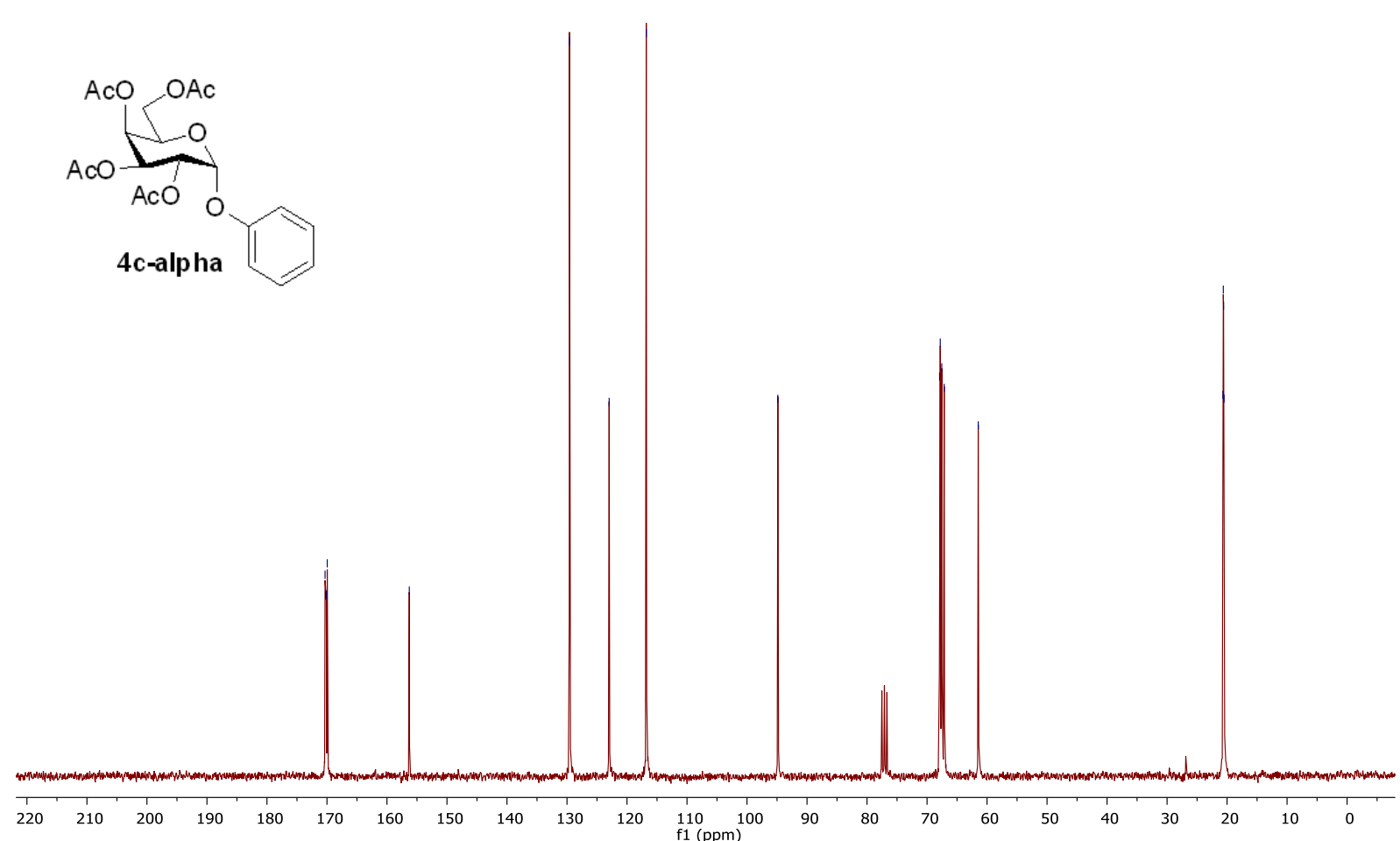
1-Phenyl-2,3,4,6-tetra-O-acetyl-b-D-galactopyranoside

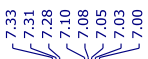

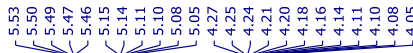

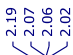

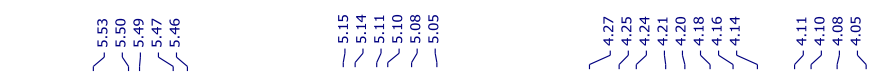
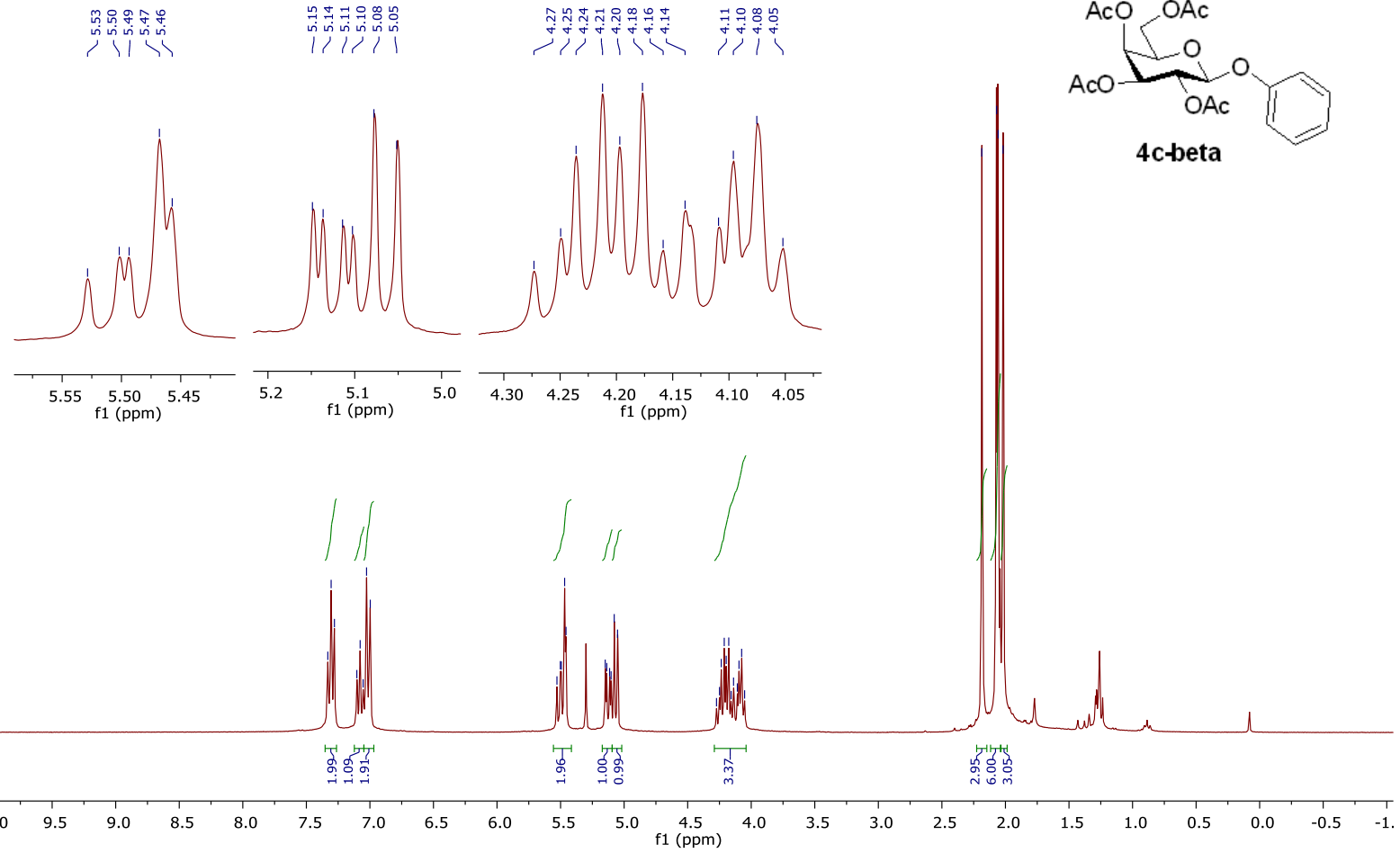

1-Phenyl-2,3,4,6-tetra-O-acetyl-b-D-galactopyranoside

Vإ

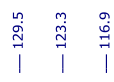

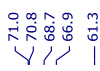

ஸें

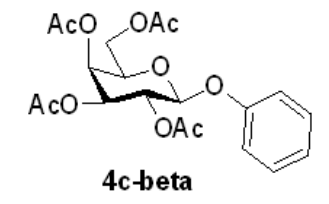

S37 
1-Phenyl-2,3,5,6-di-O-isopropylidene-a-D-mannofuranose

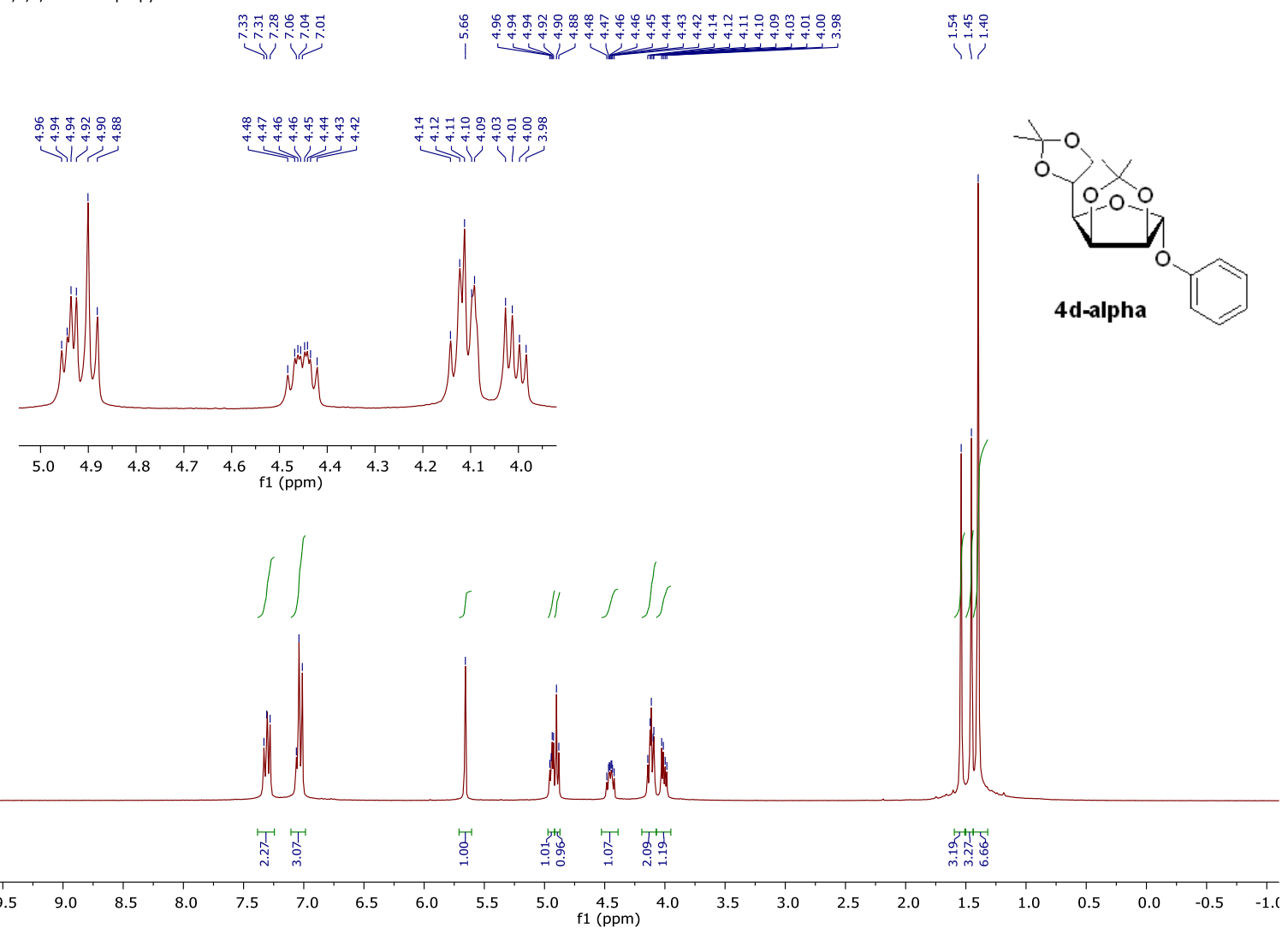

1-Phenyl-2,3,5,6-di-O-isopropylidene-a-D-mannofuranose
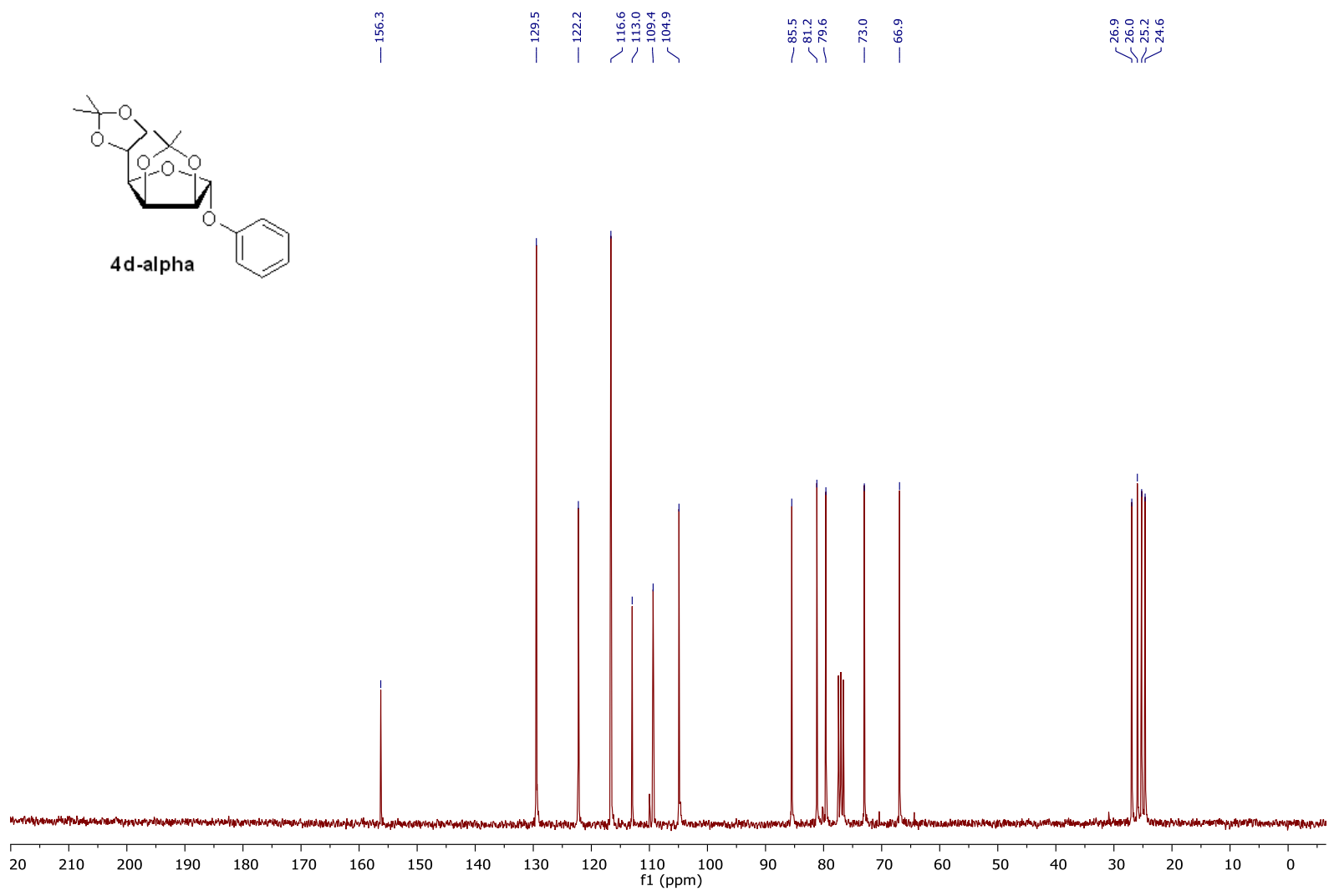

S38 
1-Phenyl-2-deoxy-,3,4,6-tri-O-acetyl-a-D-glucopyranoside

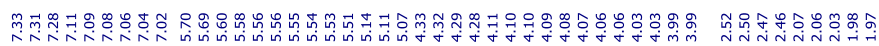

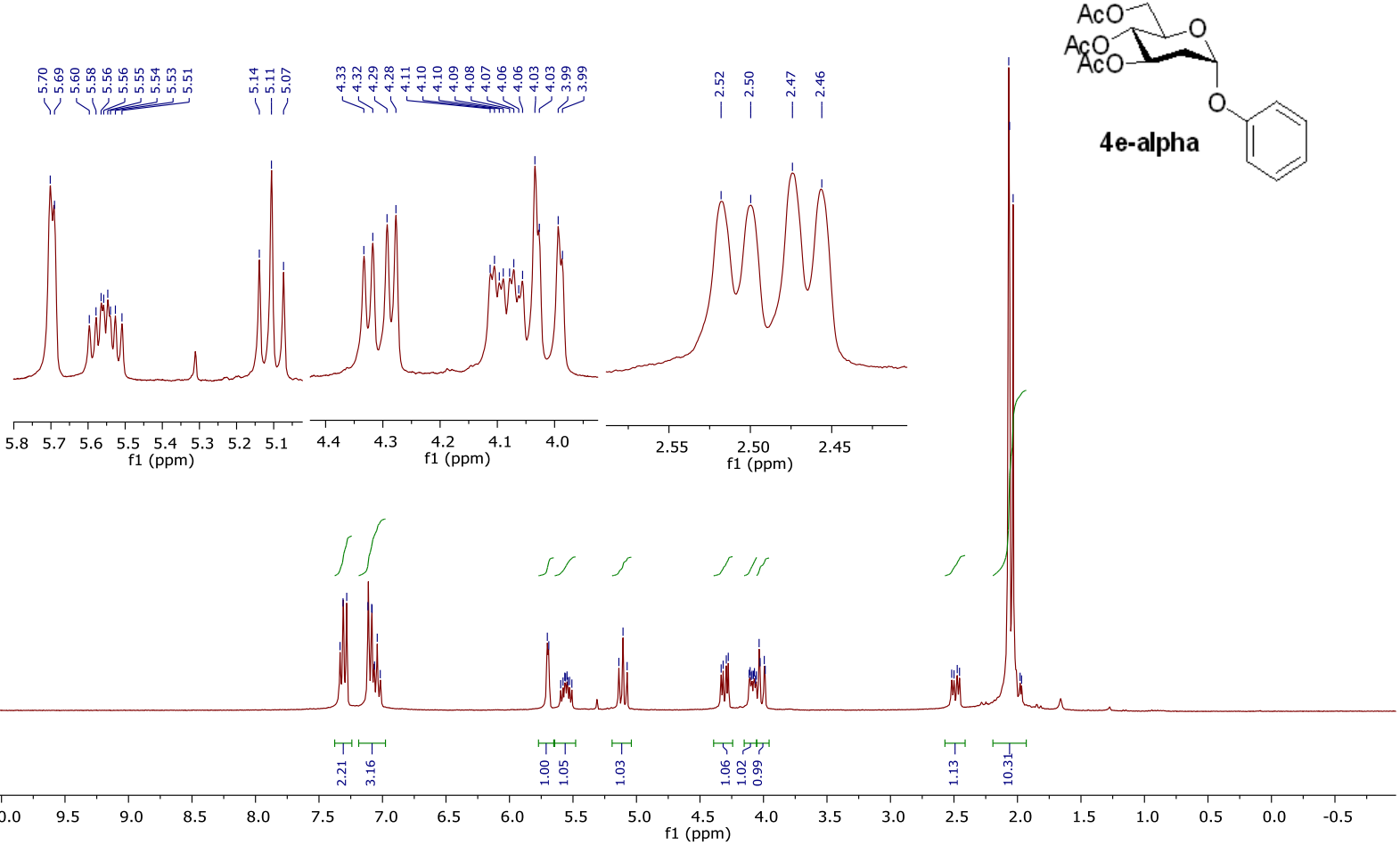

1-Phenyl-2-deoxy-,3,4,6-tri-O-acetyl-a-D-glucopyranoside

|r |

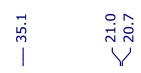

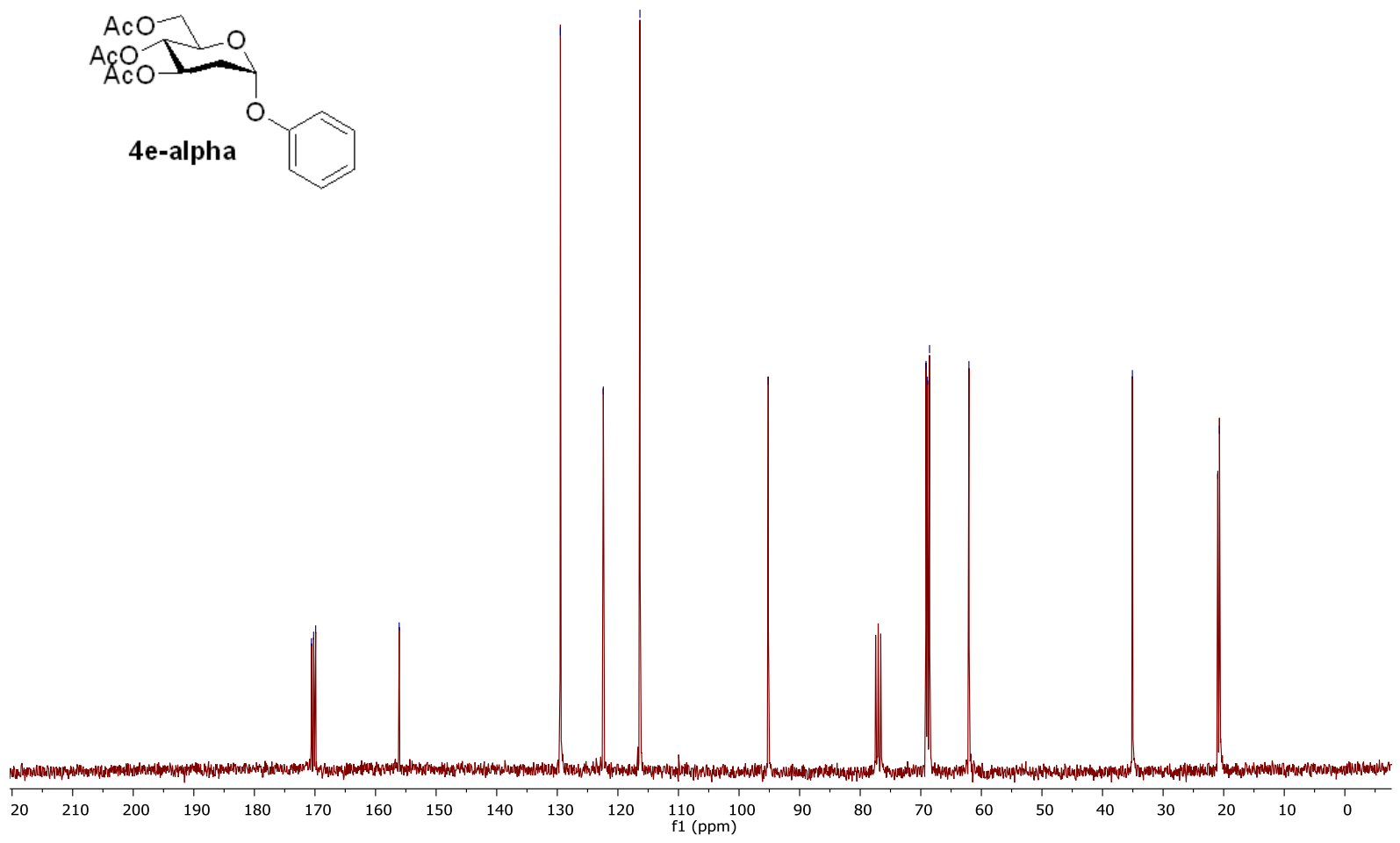

S39 
1-Phenyl-2-deoxy-,3,4,6-tri-O-acetyl-b-D-glucopyranoside

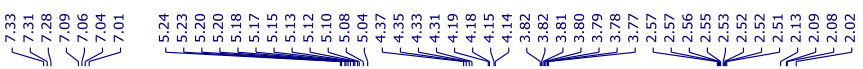

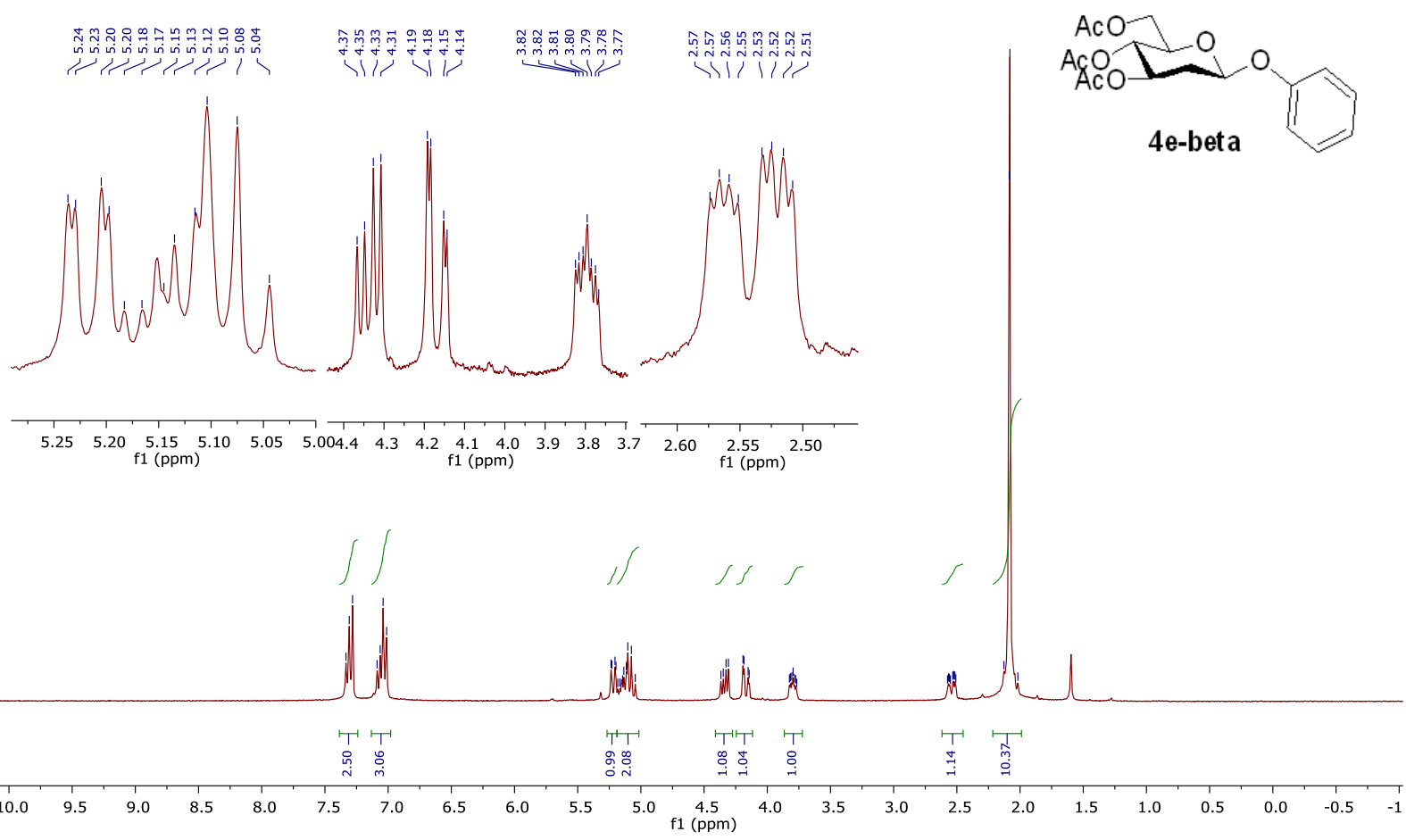

1-Phenyl-2-deoxy-,3,4,6-tri-O-acetyl-b-D-glucopyranoside

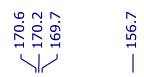

票

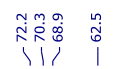

商

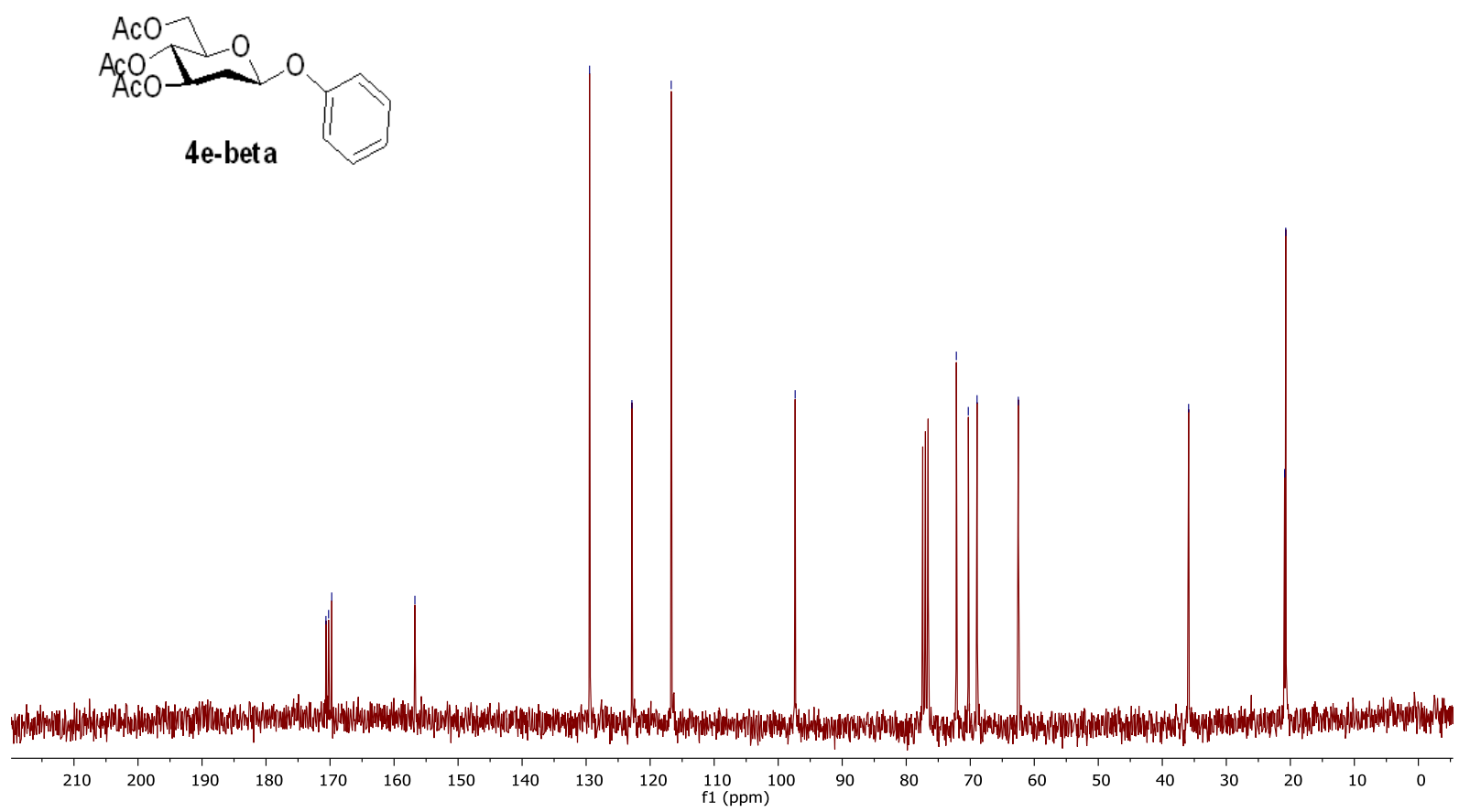

S40 


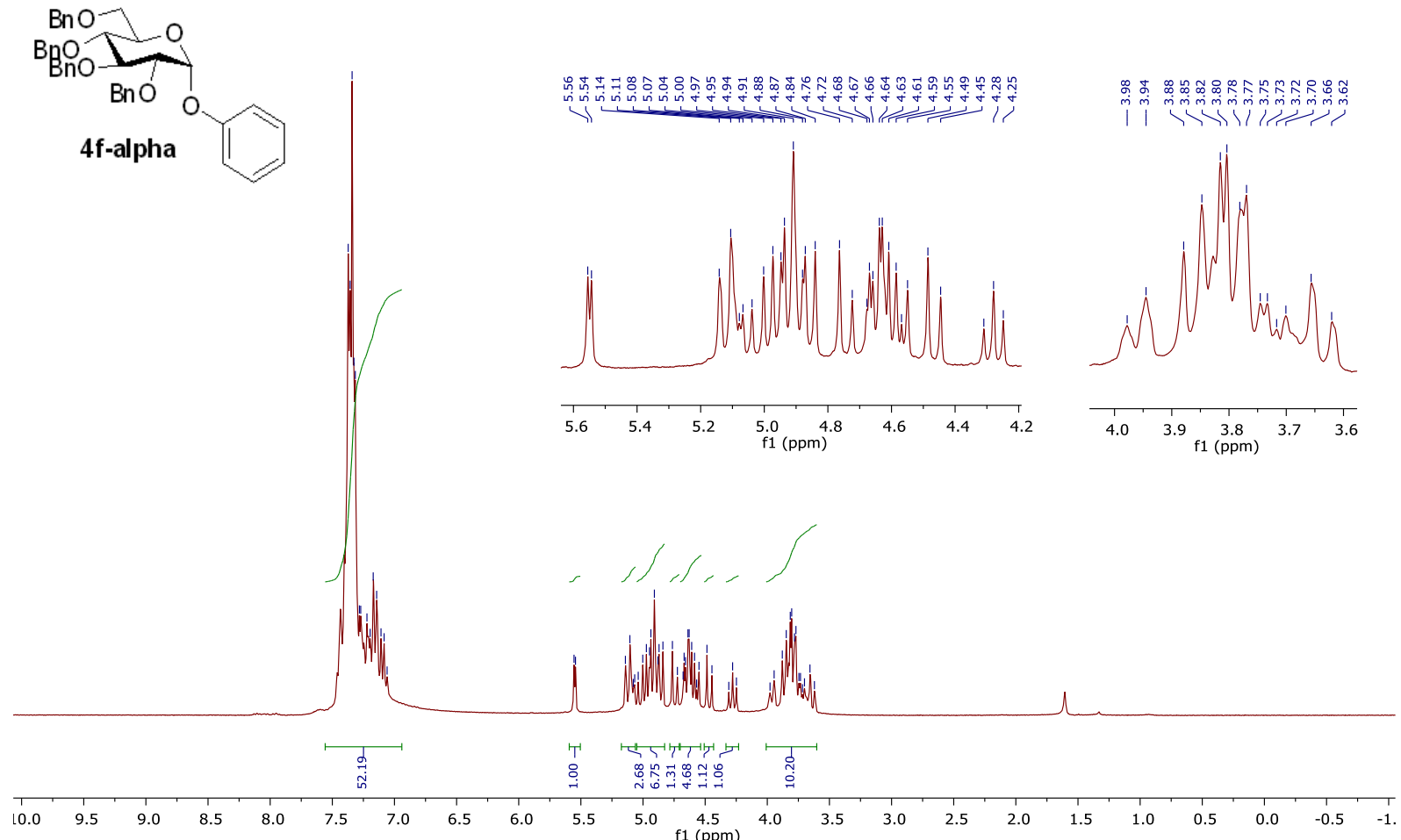

1-Phenyl-2,3,4,6-tetra-O-benzyl-D-glucopyranoside

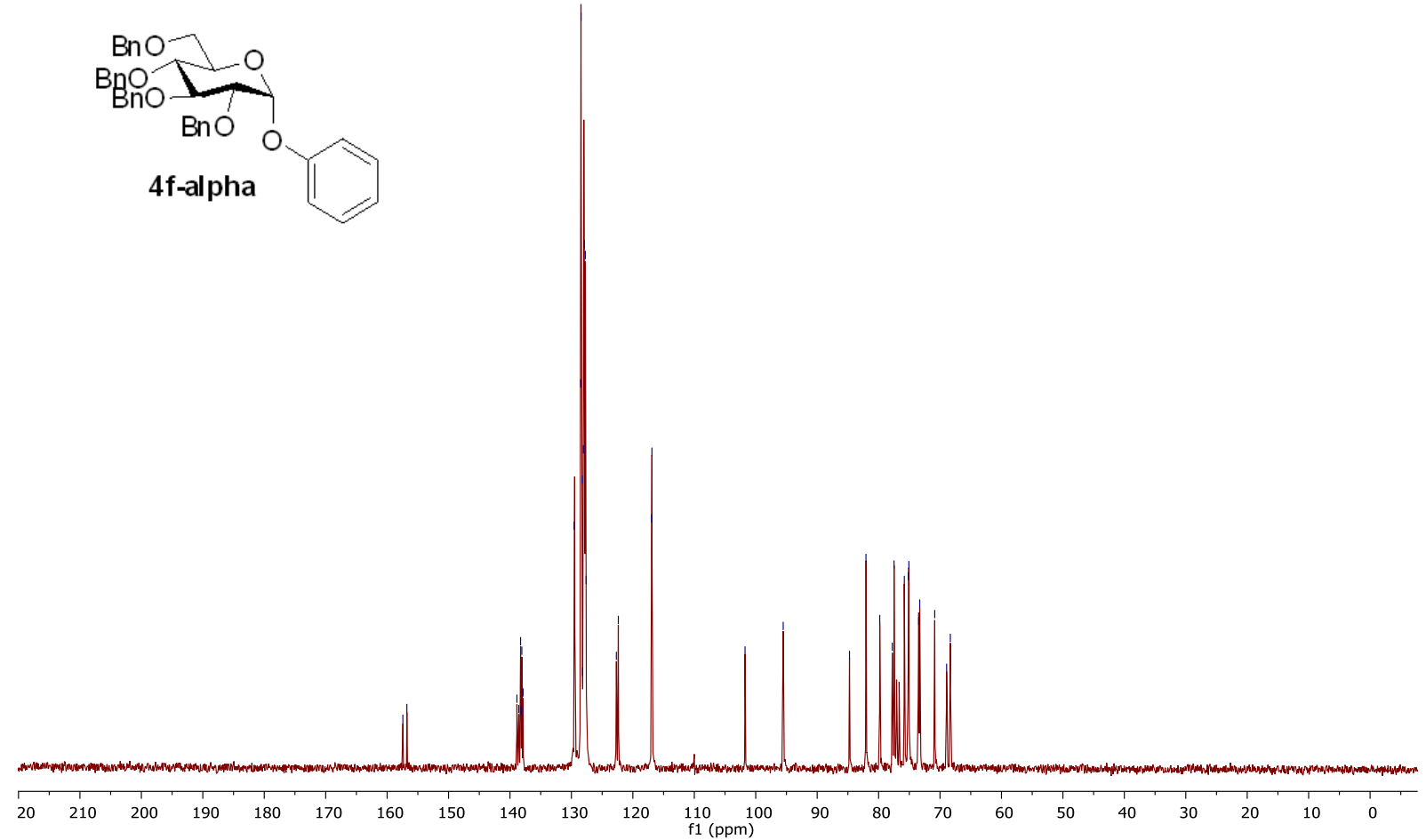


1-Phenyl-2-acetamido-3,4,6-tri-O-acetyl-2-deoxy-a-D-glucopyranos

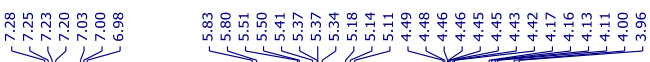

نำ

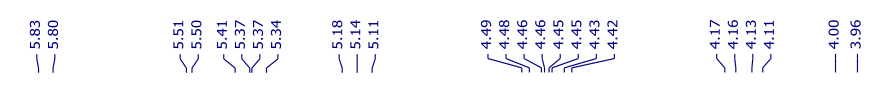
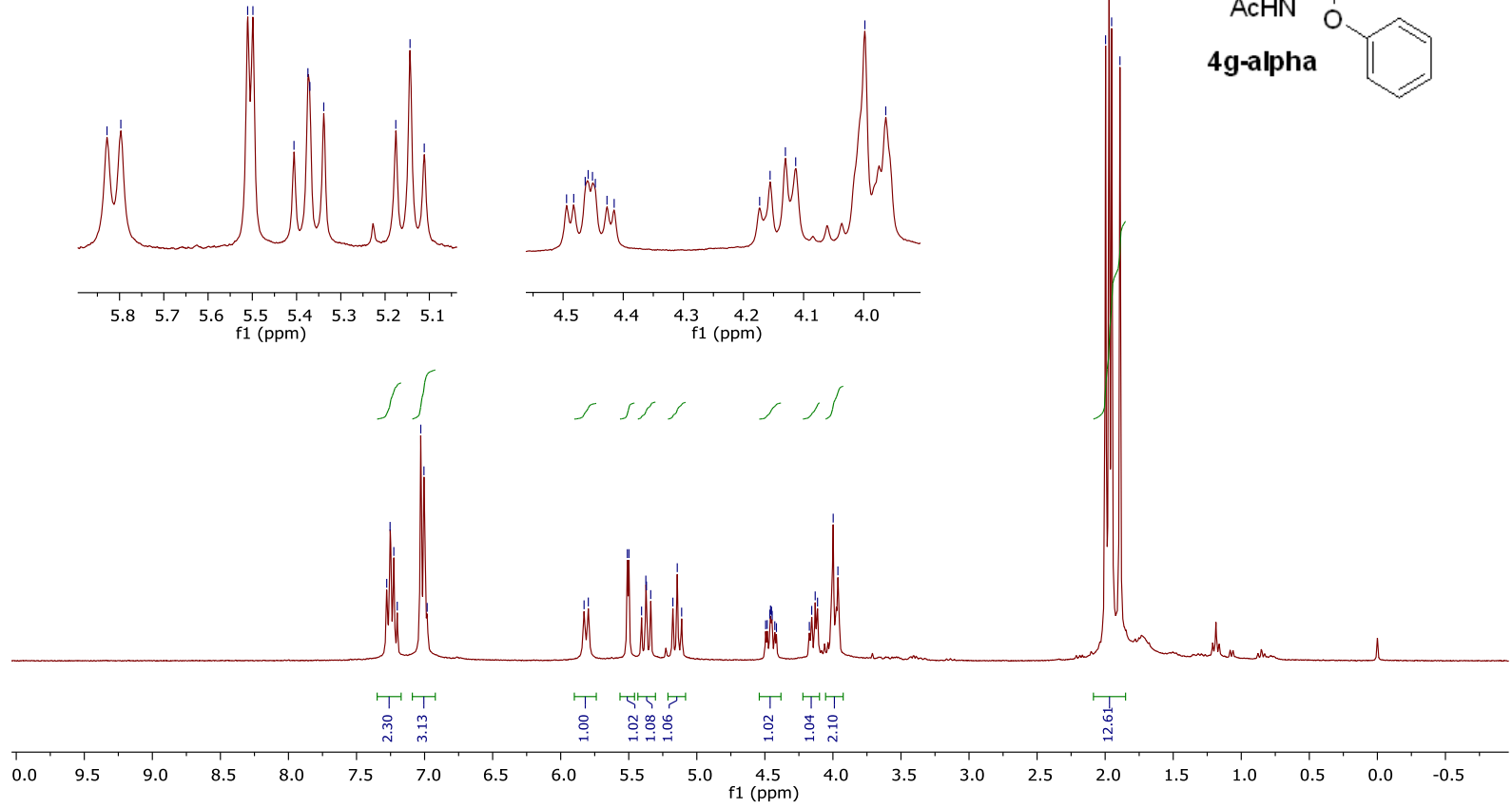

1-Phenyl-2-acetamido-3,4,6-tri-O-acetyl-2-deoxy-a-D-glucopyranose

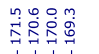

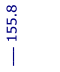

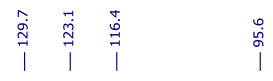

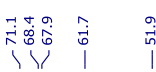

พิ่าำ
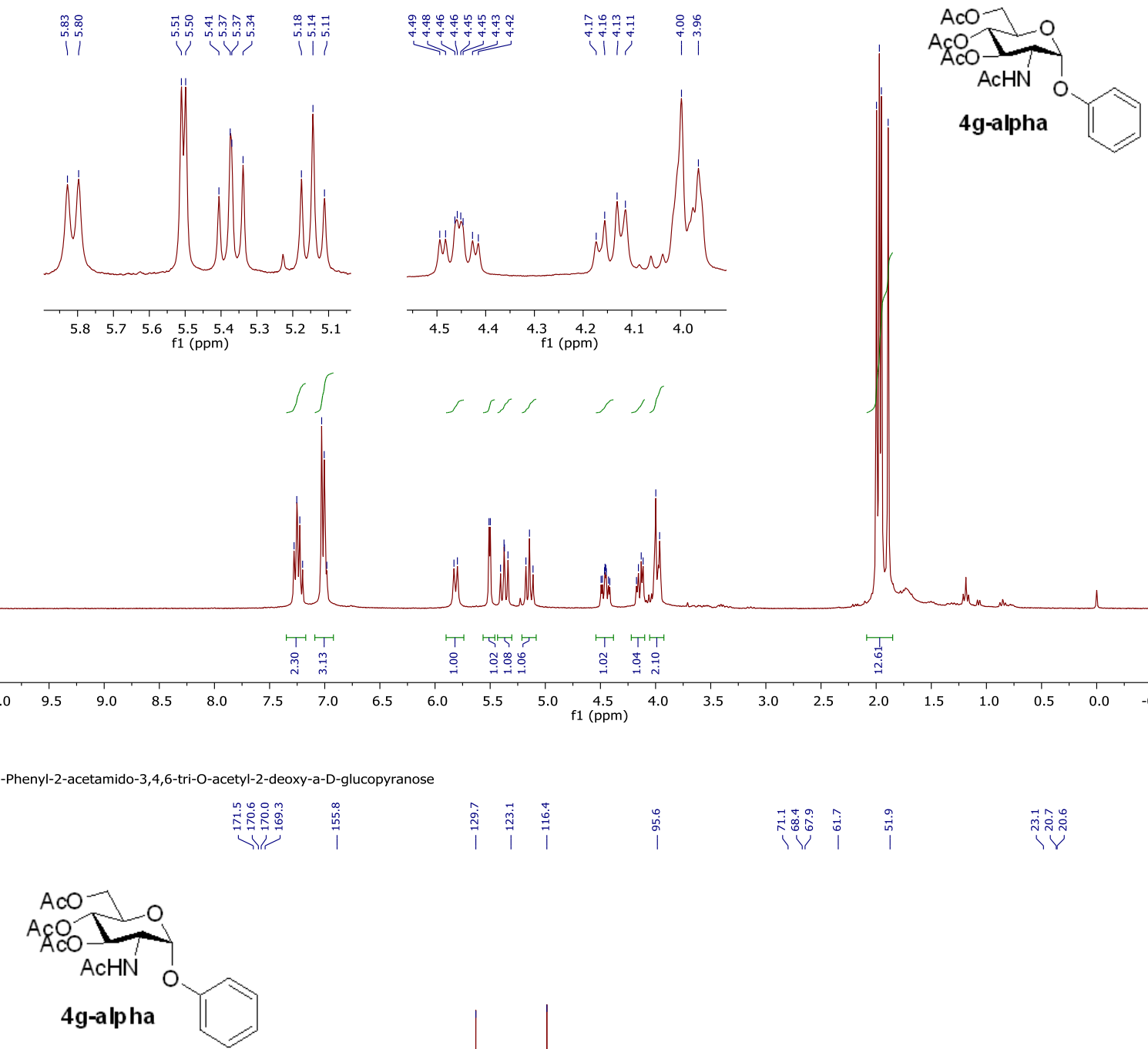

4g-alpha 

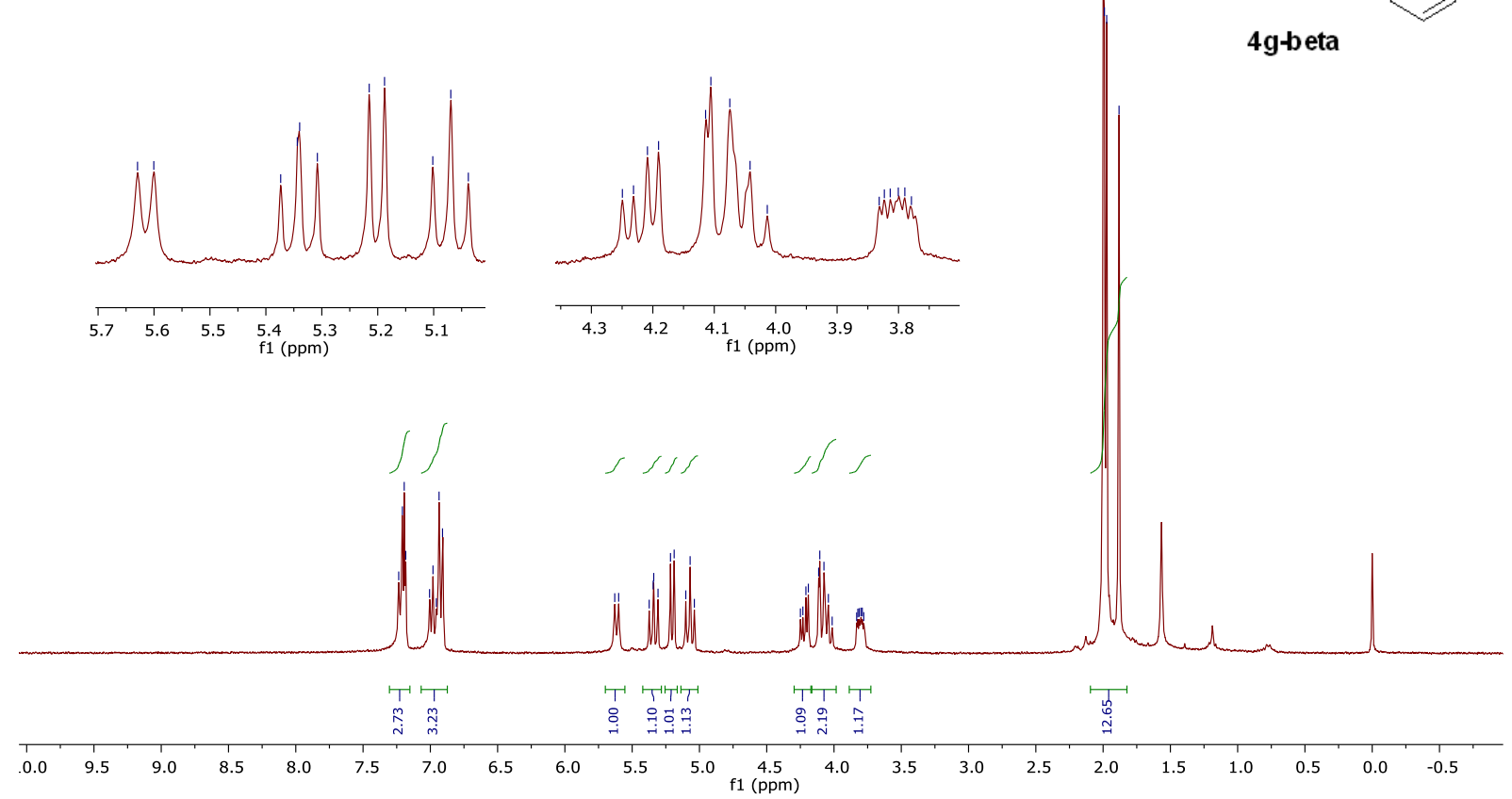

1-Phenyl-2-acetamido-3,4,6-tri-O-acetyl-2-deoxy-b-D-glucopyranose

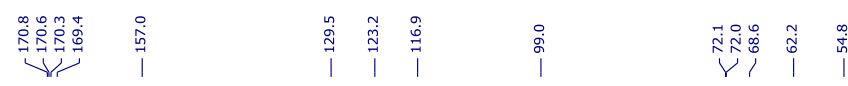
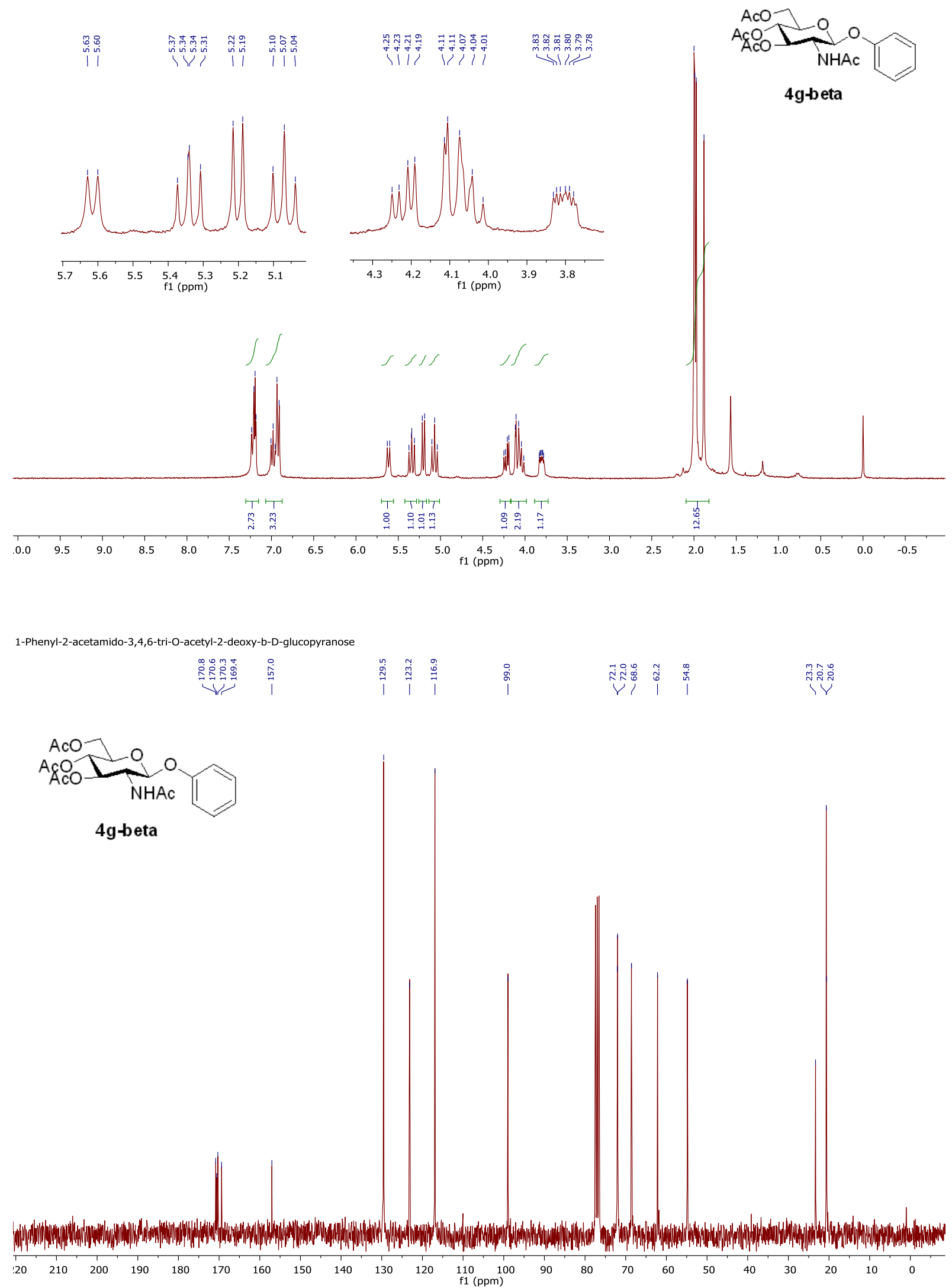


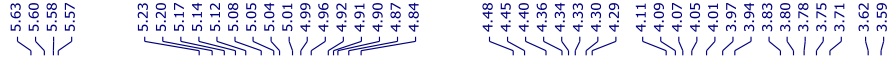

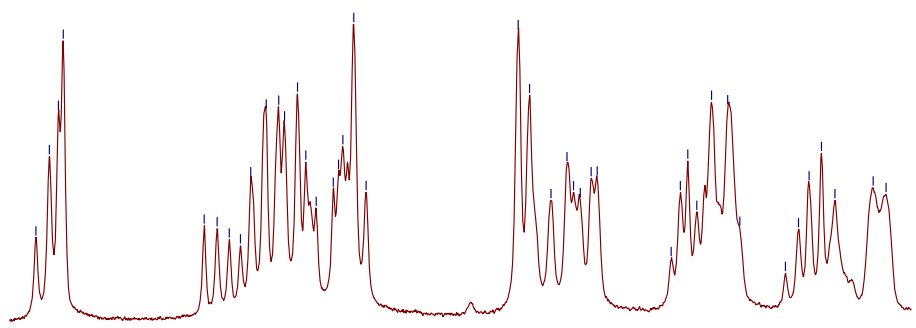

AcO $\frac{A_{A C O} A C O}{A C O}$

$4 \mathbf{i}, 77 \%(\beta \alpha / \beta \beta=1.5: 1)$

$\begin{array}{lllllllllllllllllllll}5.6 & 5.5 & 5.4 & 5.3 & 5.2 & 5.1 & 5.0 & 4.9 & 4.8 & 4.7 & 4.6 & 4.5 & 4.4 & 4.3 & 4.2 & 4.1 & 4.0 & 3.9 & 3.8 & 3.7 & 3.6\end{array}$ f1 $(\mathrm{ppm})$

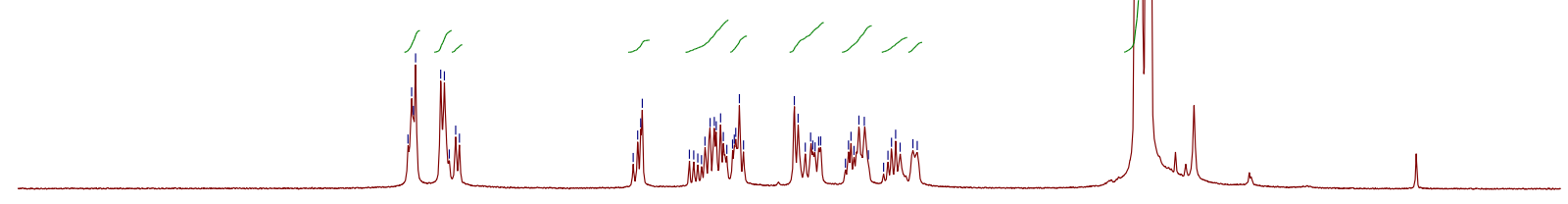

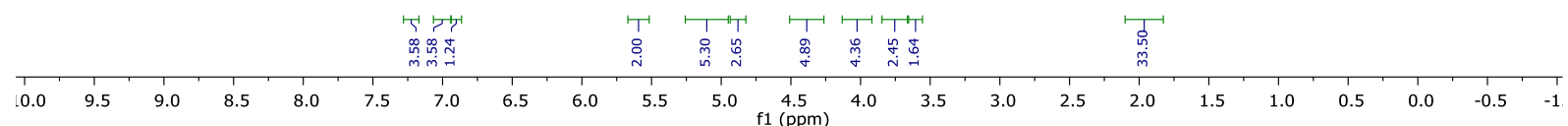

1-phenyl-2,3,6,2',3',4',6'-Hepta-O-acetylcellobiose

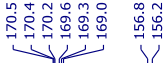

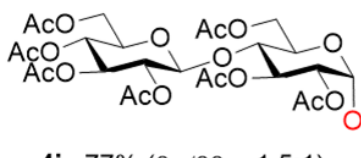

$4 \mathbf{i}, \quad 77 \%(\beta \alpha / \beta \beta=1.5: 1)$
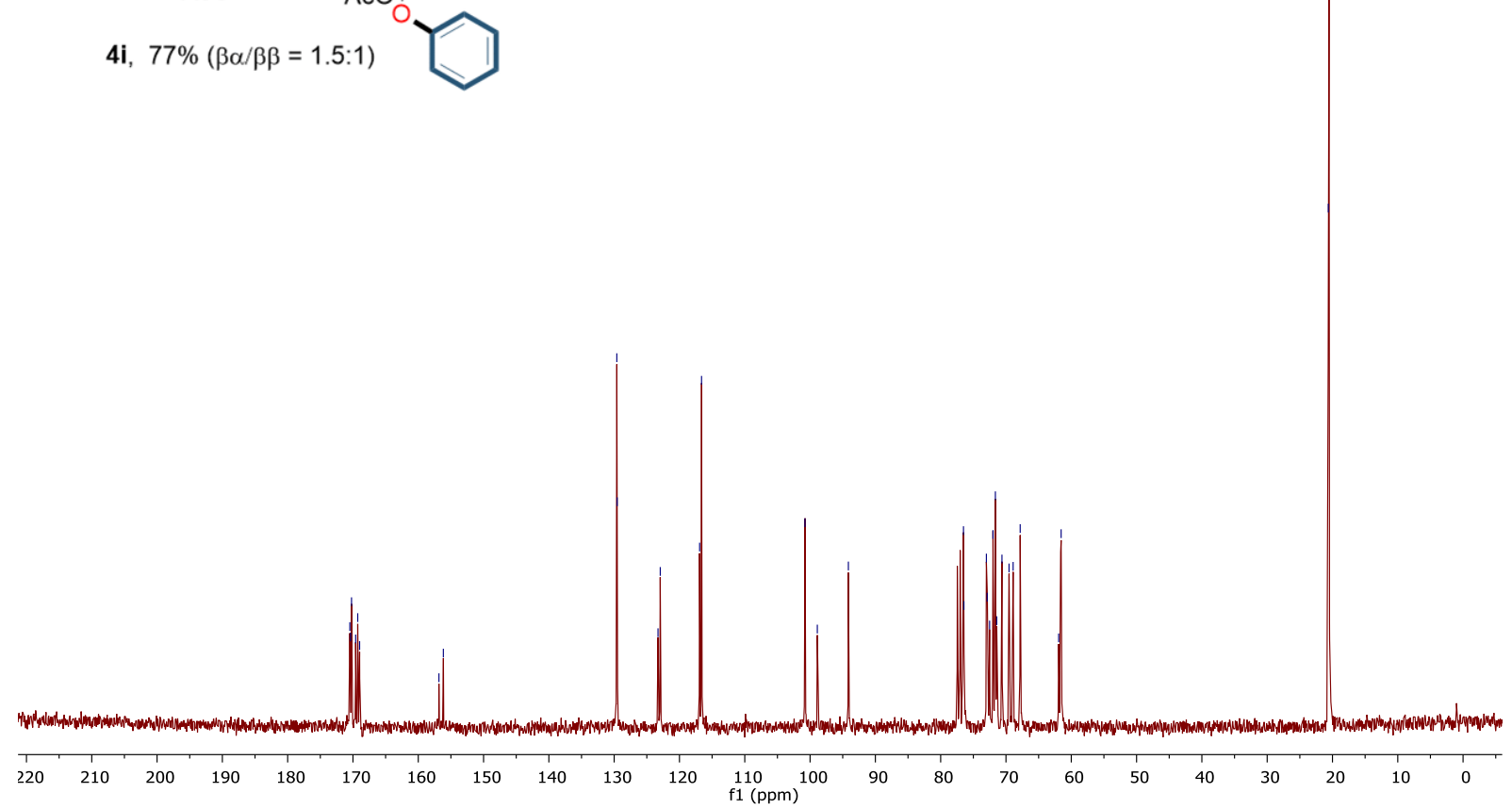


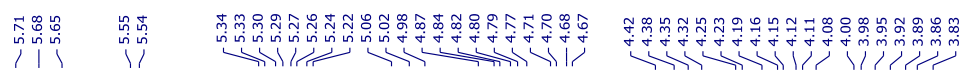

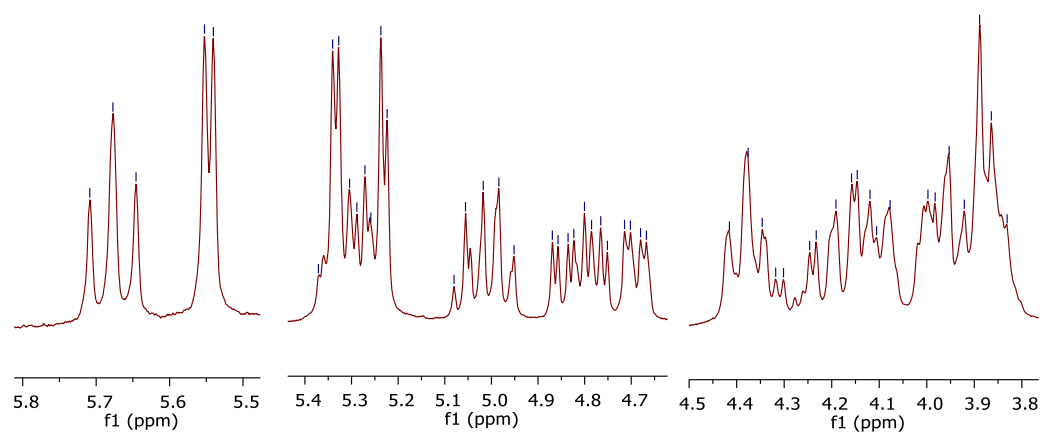

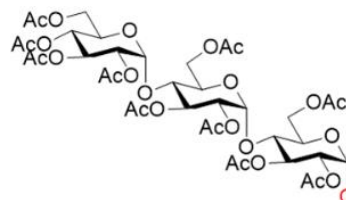

4j. $76 \%(\alpha \alpha \alpha / \alpha \alpha \beta=1.6: 1)$

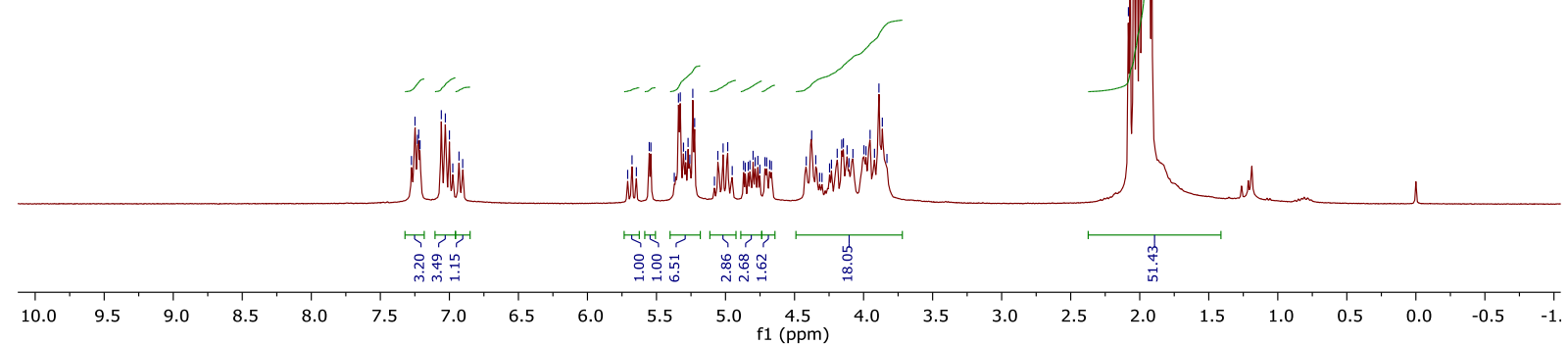

1-Phenyl-(4-O-(4-O-(2,3,4,6-tetra-O-acetyt-D-glucopyranosyl)-2,3,6-tri-O-acetyla-D-glucopyranosyl))-2,3,6-tri-O-acetyl-D-glucopyranose

等

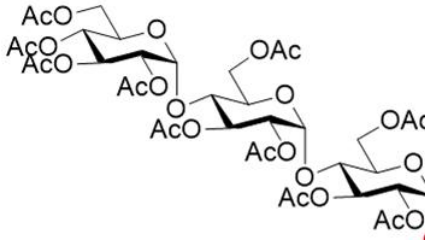

4j, $76 \%(\alpha \alpha \alpha / \alpha \alpha \beta=1.6: 1)$

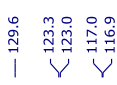

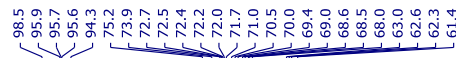

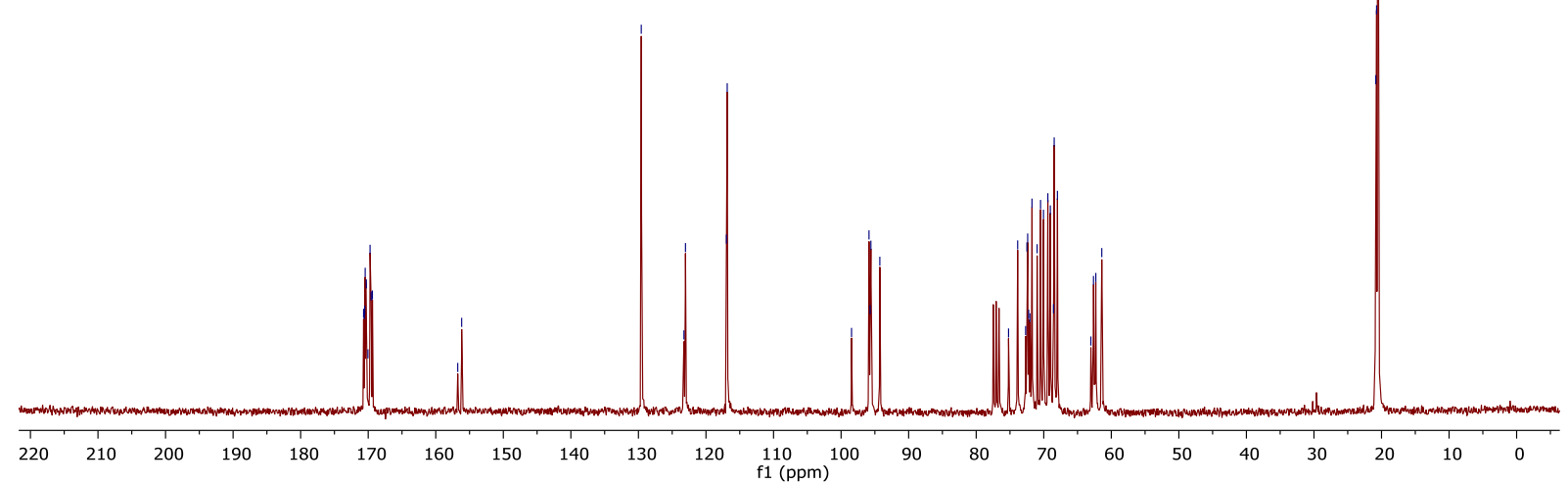




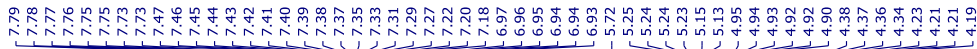

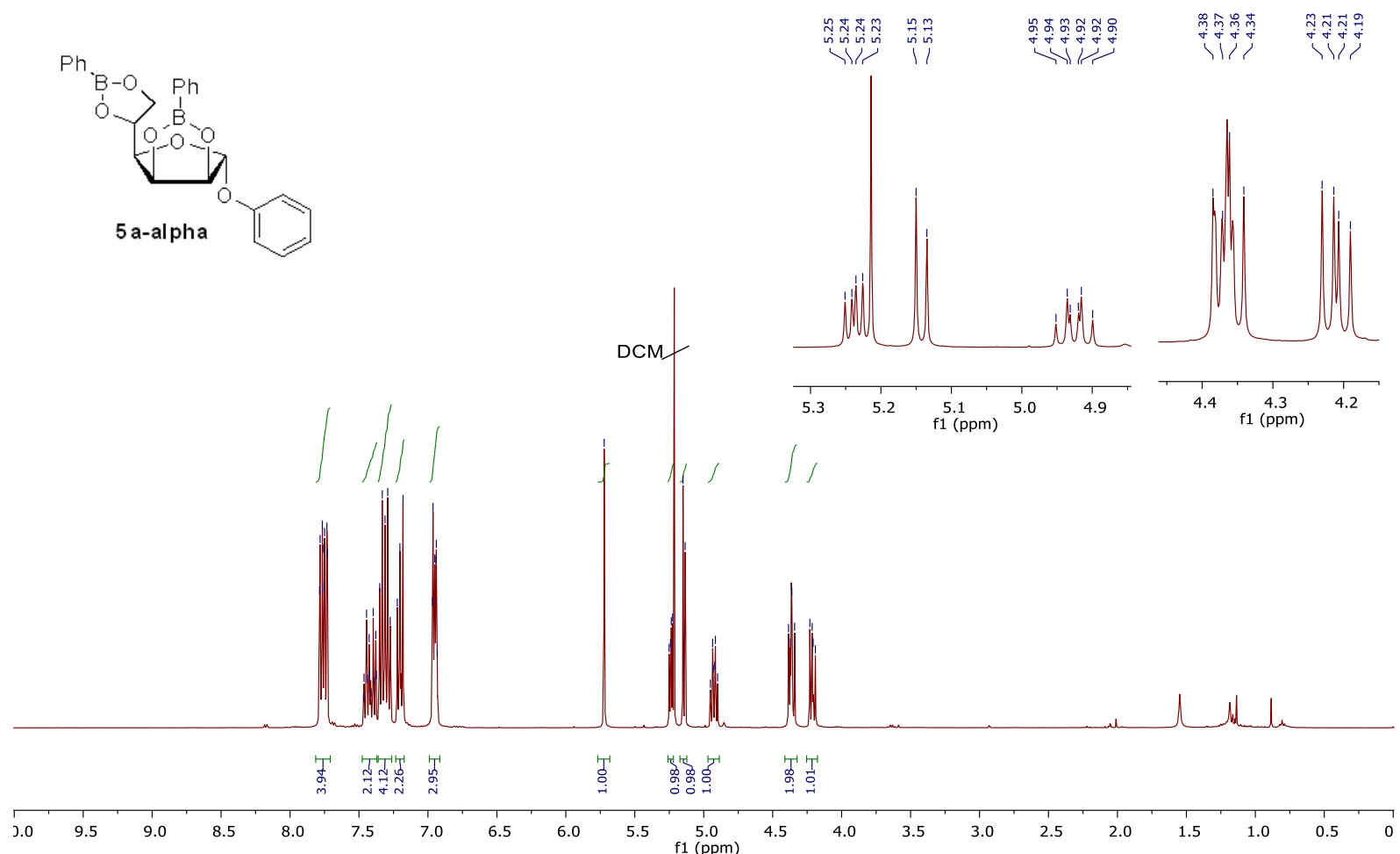

(3aS,4R,6R,6aR)-4-phenoxy-2-phenyl-6-(2-phenyl-1,3,2-dioxaborolan-4-yl)tetrahydrofuro[3,4-d][1,3,2]dioxaborole.

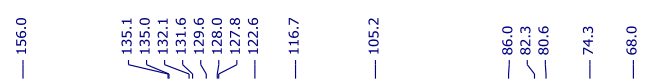

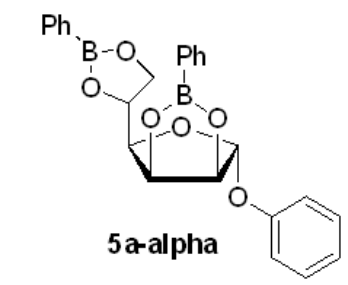




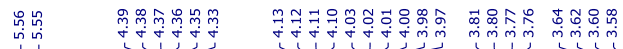

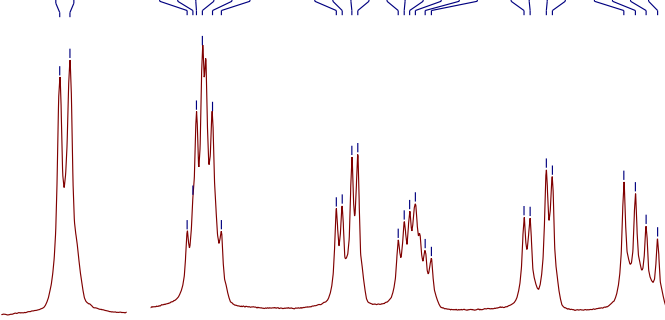

$\begin{array}{ll}5.58 & 5.52 \\ \mathrm{f} 1(\mathrm{ppm})\end{array}$
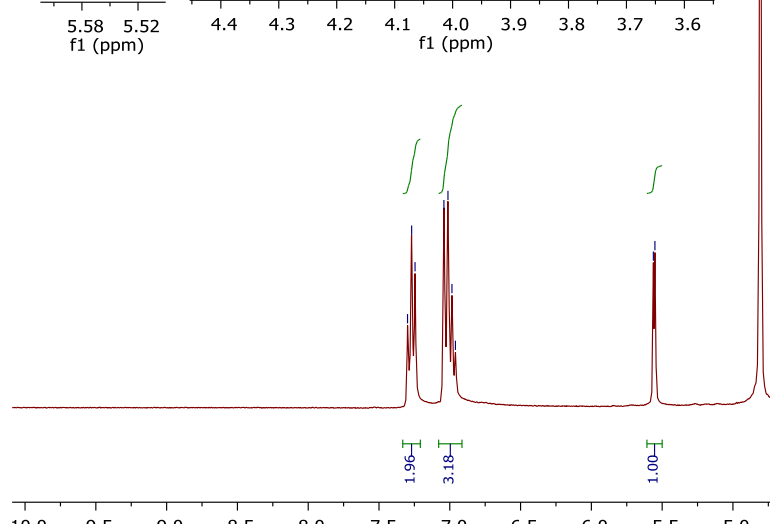

票票

蒋

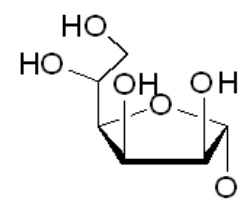

6a-alpha

1-Phenyl-a-D-mannofuranose

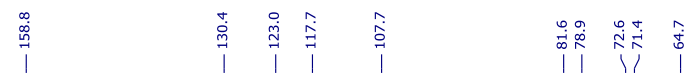

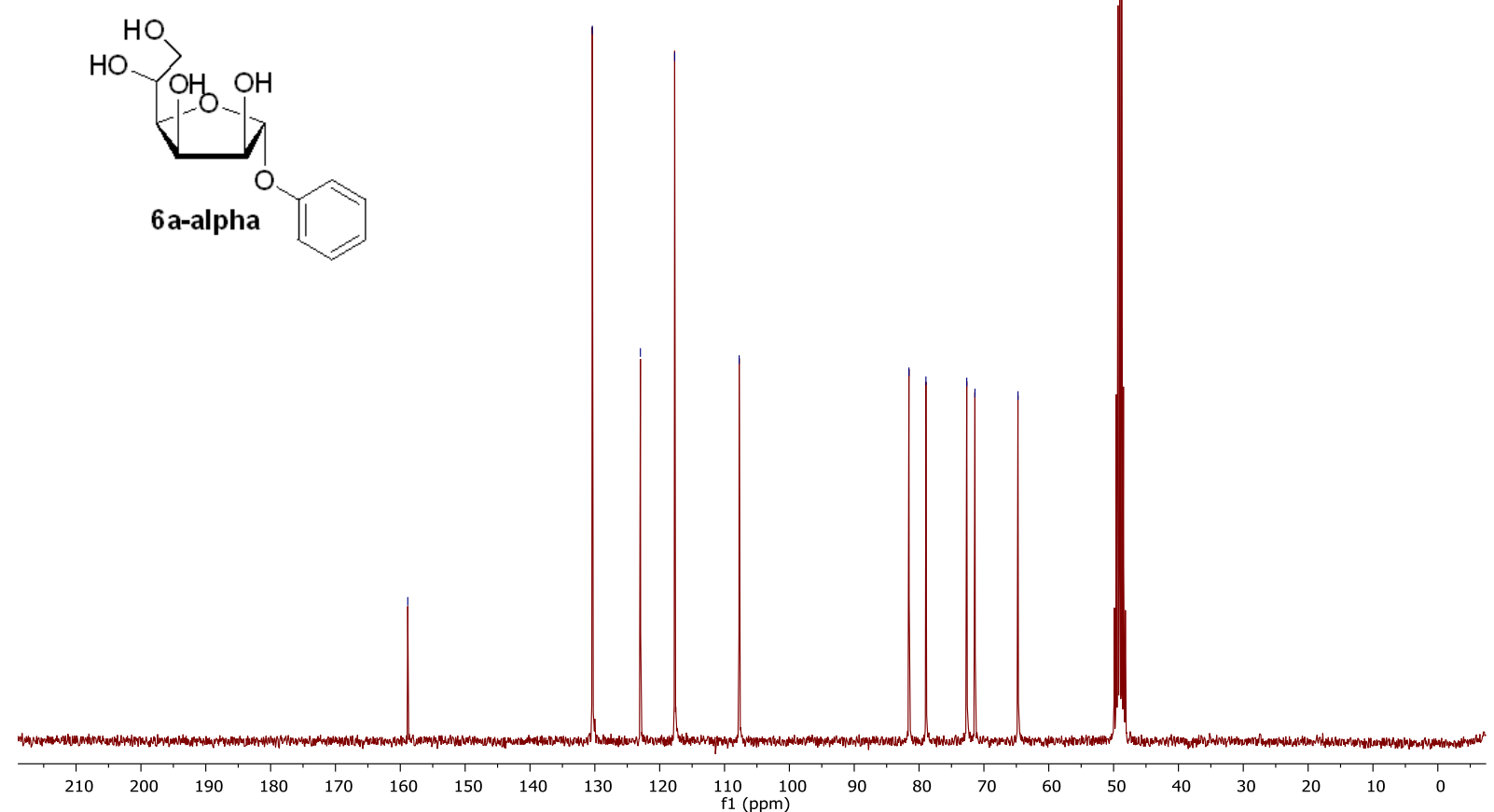


NMR data of synthesized starting materials $1 \mathrm{~b}, 1 \mathrm{~d}, 1 \mathrm{f}, 1 \mathrm{~h}, 1 \mathrm{i}$ and $1 \mathrm{j}$

2,3,4,6-tetra-a-O-acetyl-D-glucopyranose

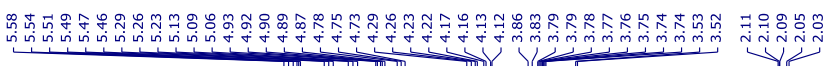

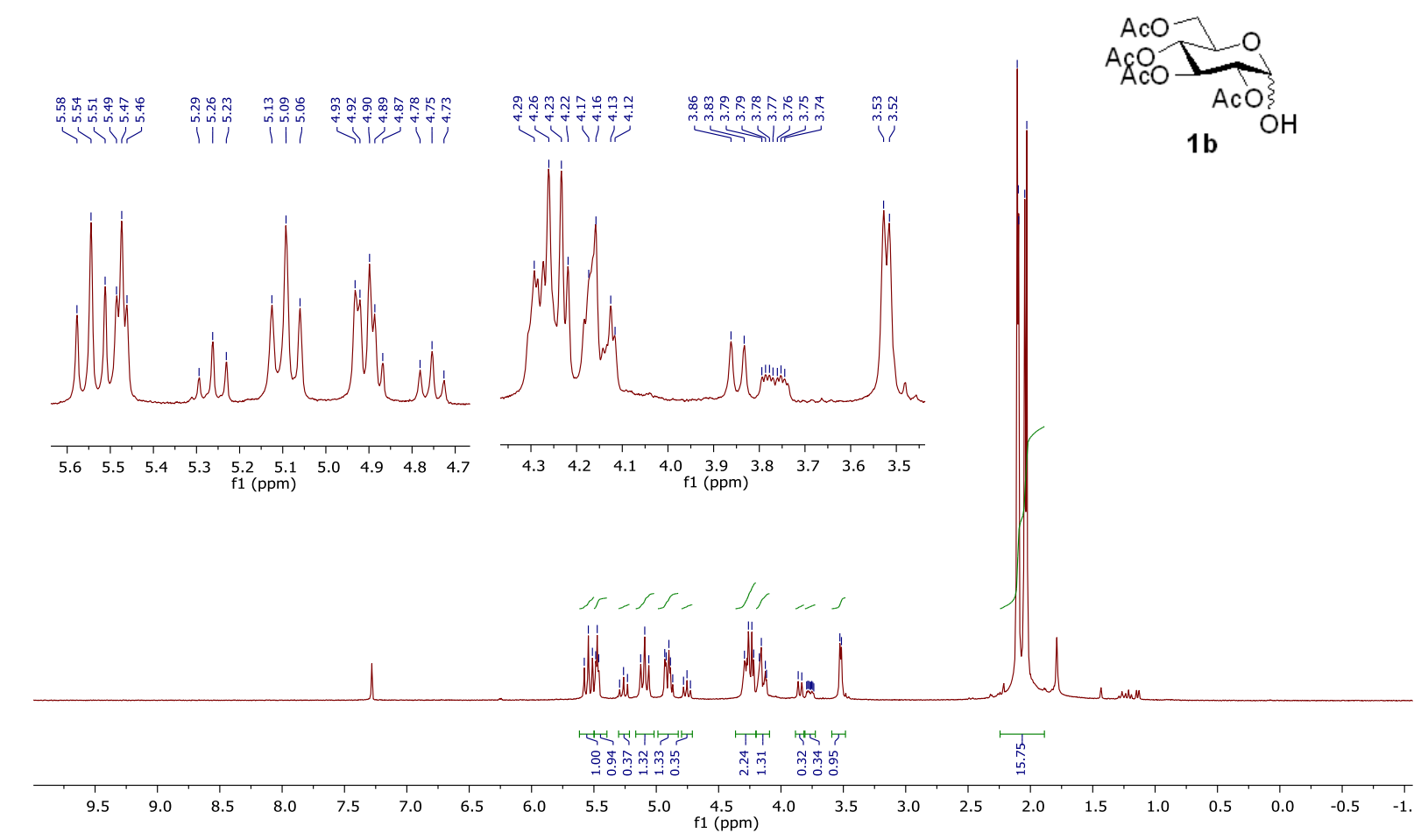




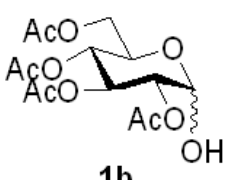

1b

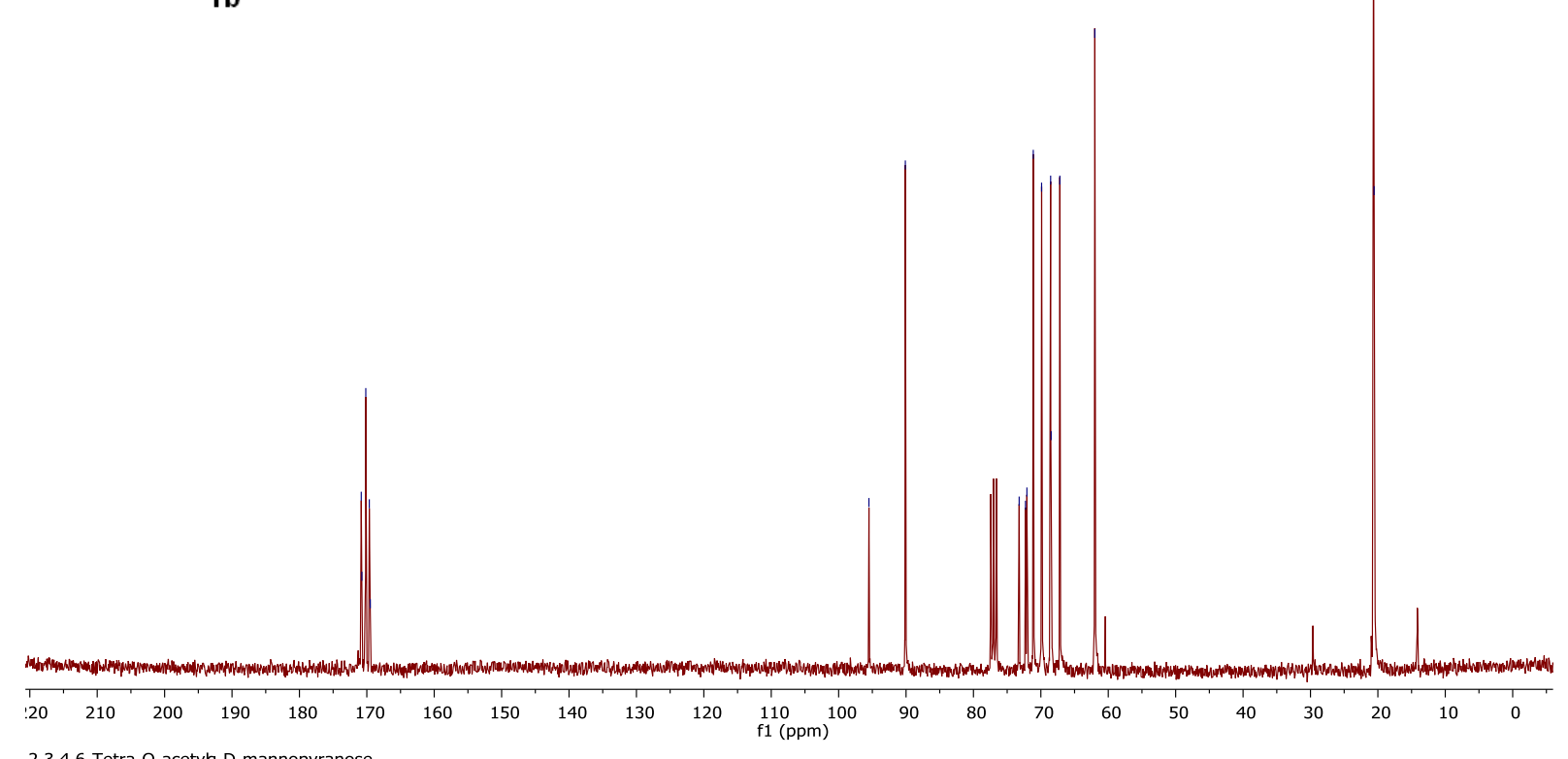
2,3,4,6-Tetra-O-acetyla-D-mannopyranose
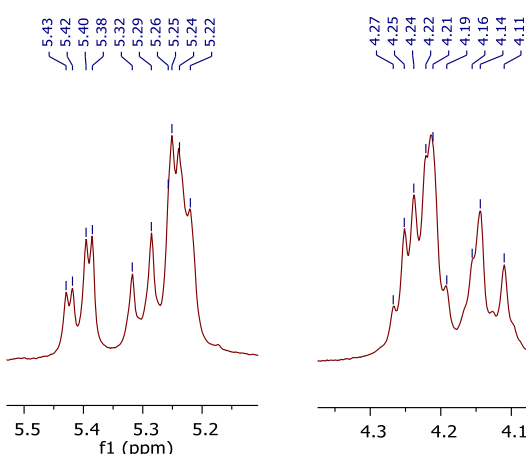

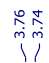
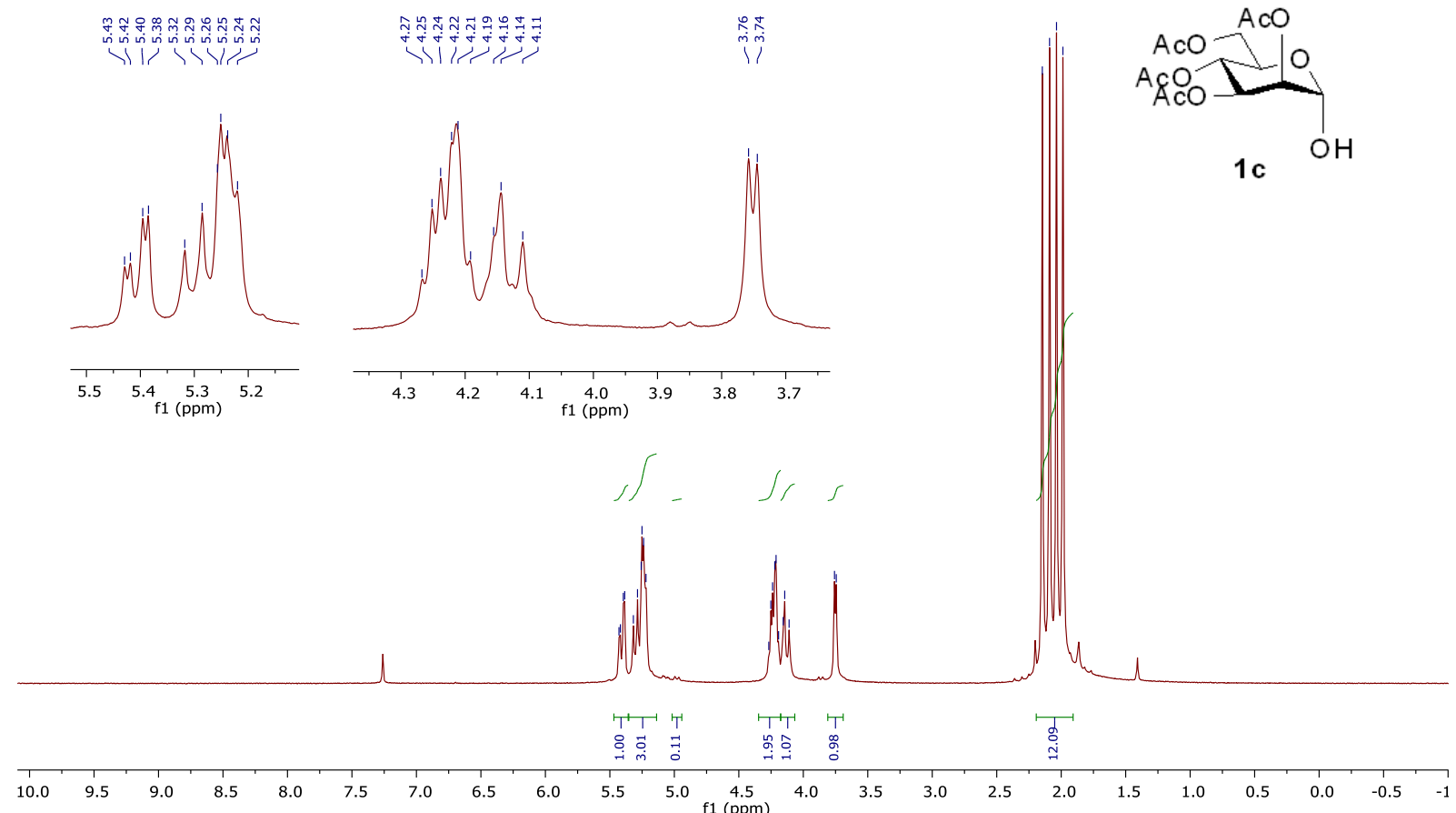

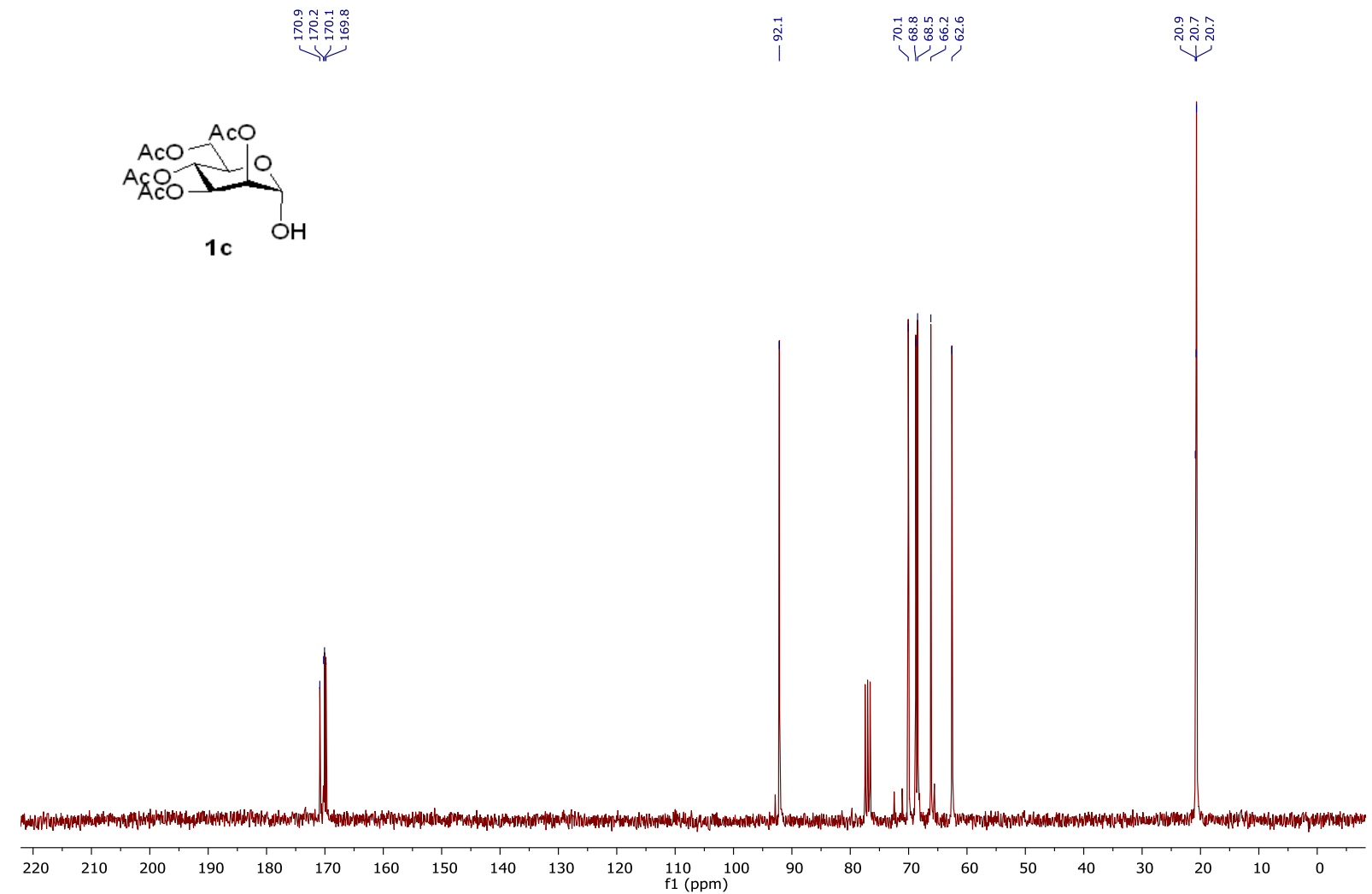

2,3,4,6-Tetra-O-acetyl-D-galactopyranose

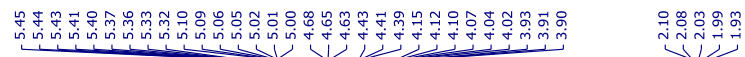

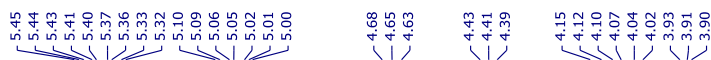
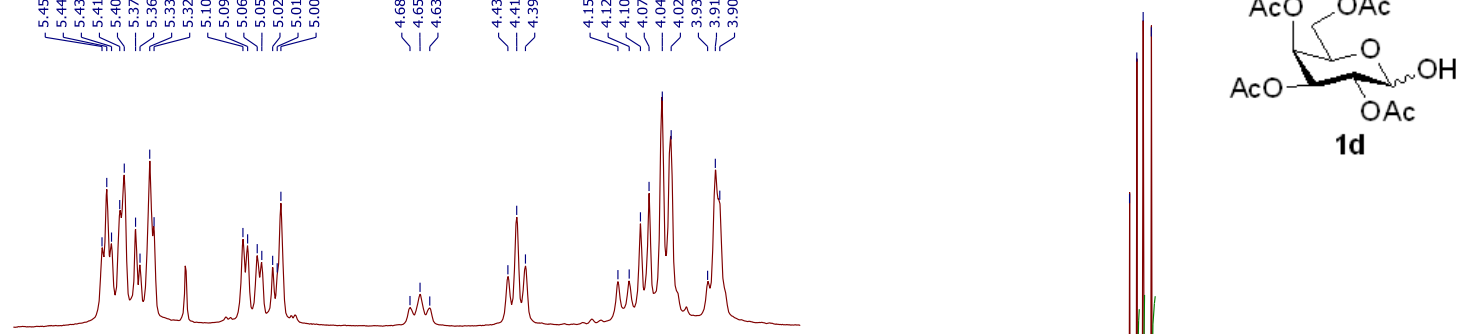

1d

$\begin{array}{lllllllllllllllllllllllll}5.6 & 5.5 & 5.4 & 5.3 & 5.2 & 5.1 & 5.0 & 4.9 & 4.8 & 4.7 & 4.6 & 4.5 & 4.4 & 4.3 & 4.2 & 4.1 & 4.0 & 3.9 & 3.8 & 3.7\end{array}$ f1 ( $\mathrm{ppm})$
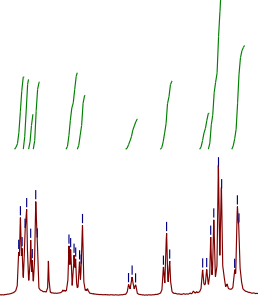

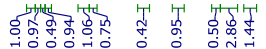

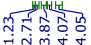

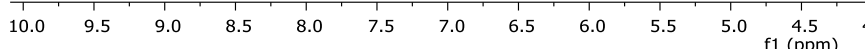


2,3,4,6-Tetra-O-acetyl-D-galactopyranose

\section{0}

10.0.

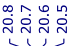

${ }_{{ }_{1 d} A c}^{A c O}$

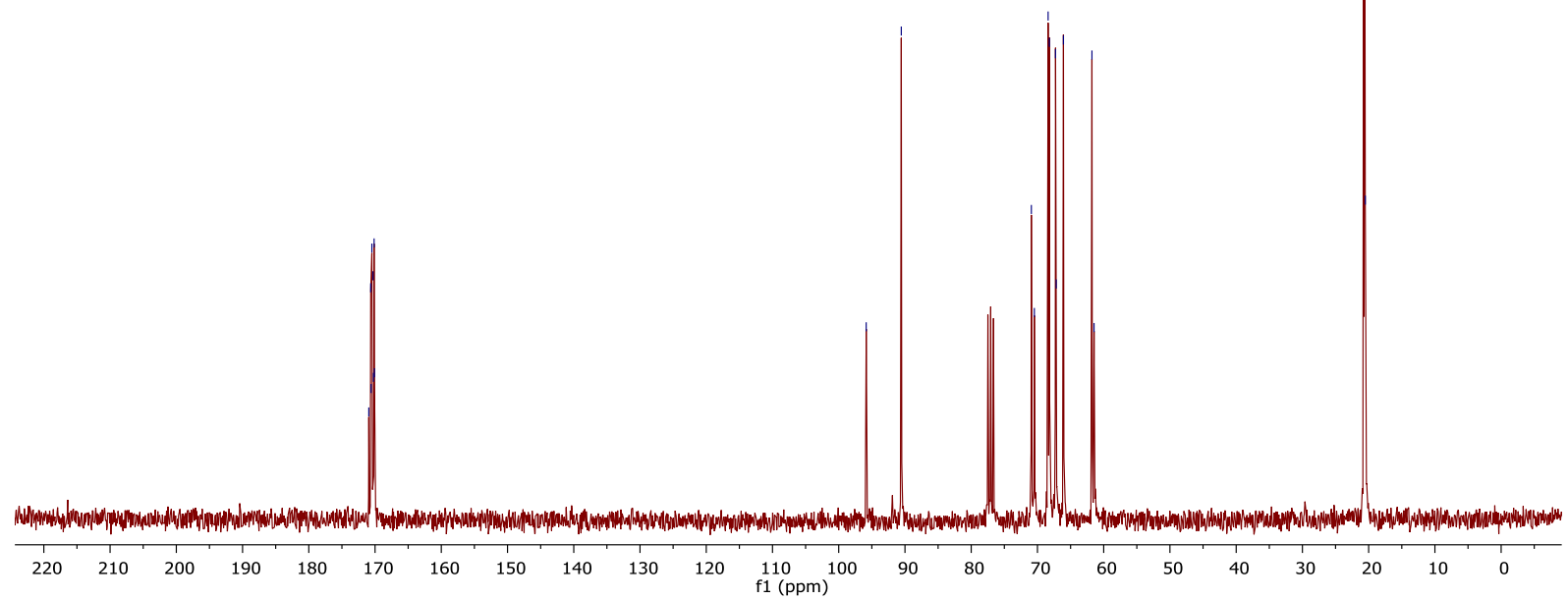

2-Acetamido-3,4,6-tri-O-acetyl-2-deoxya-D-glucopyranose
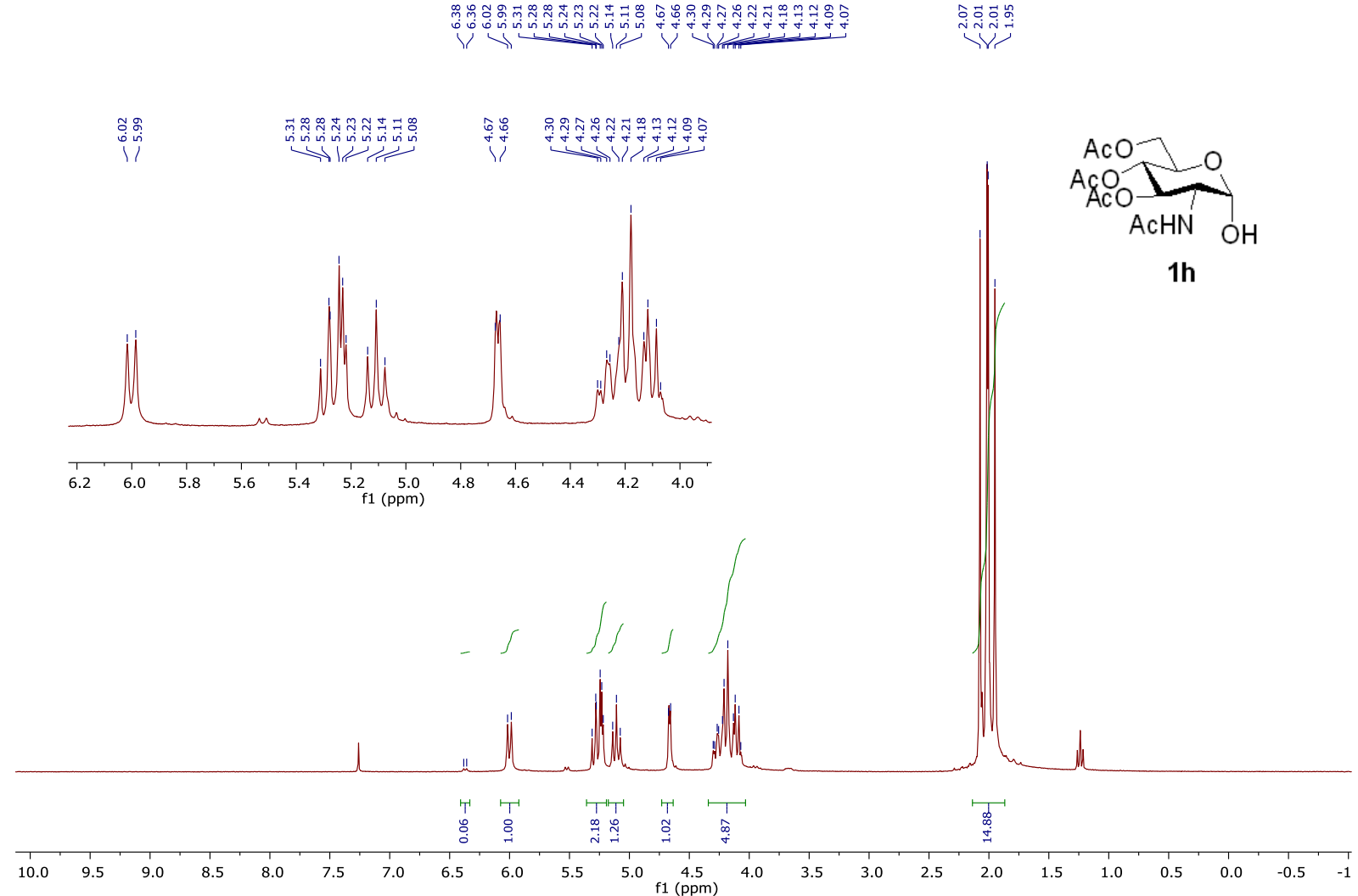


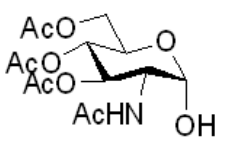

1h

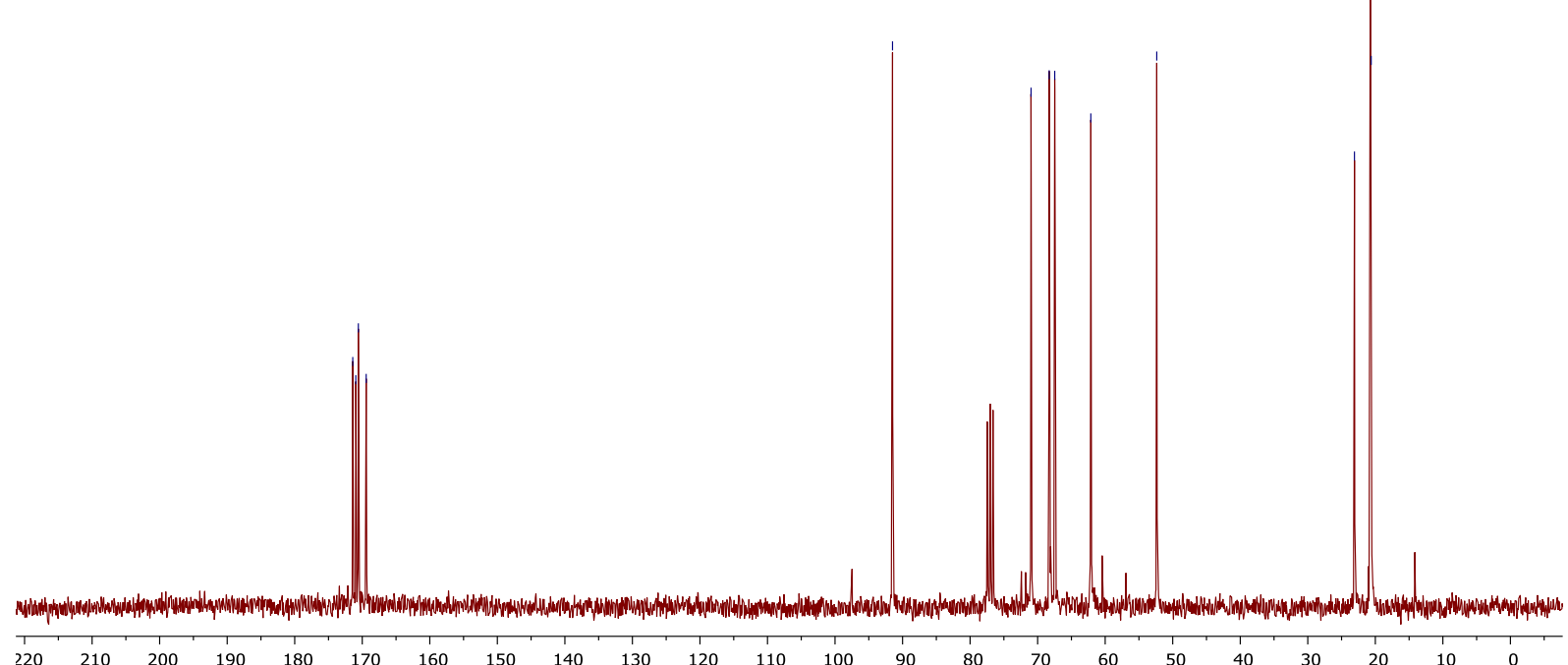
$\begin{array}{llllll}220 & 210 & 200 & 190 & 180 & 170\end{array}$

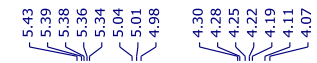
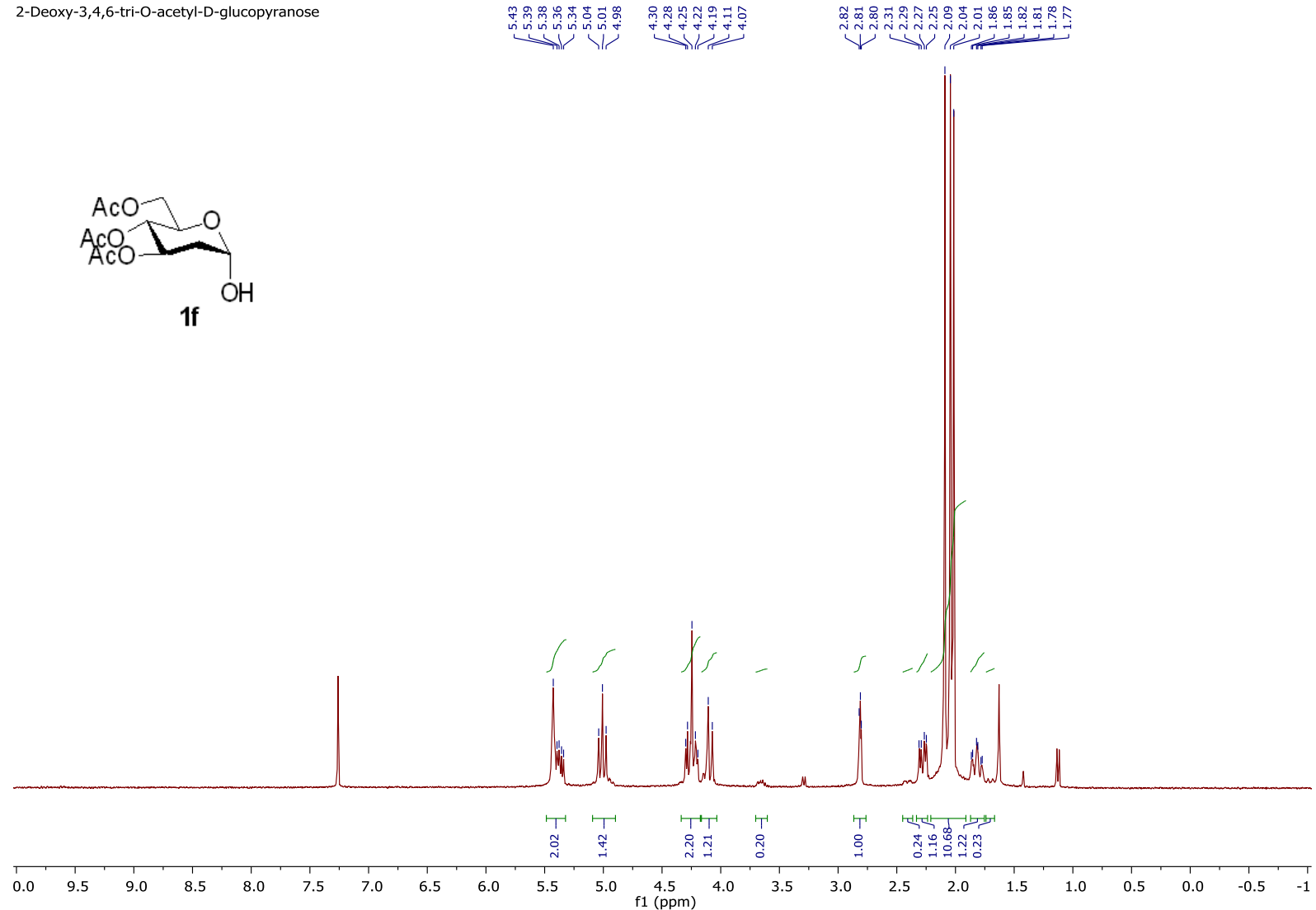

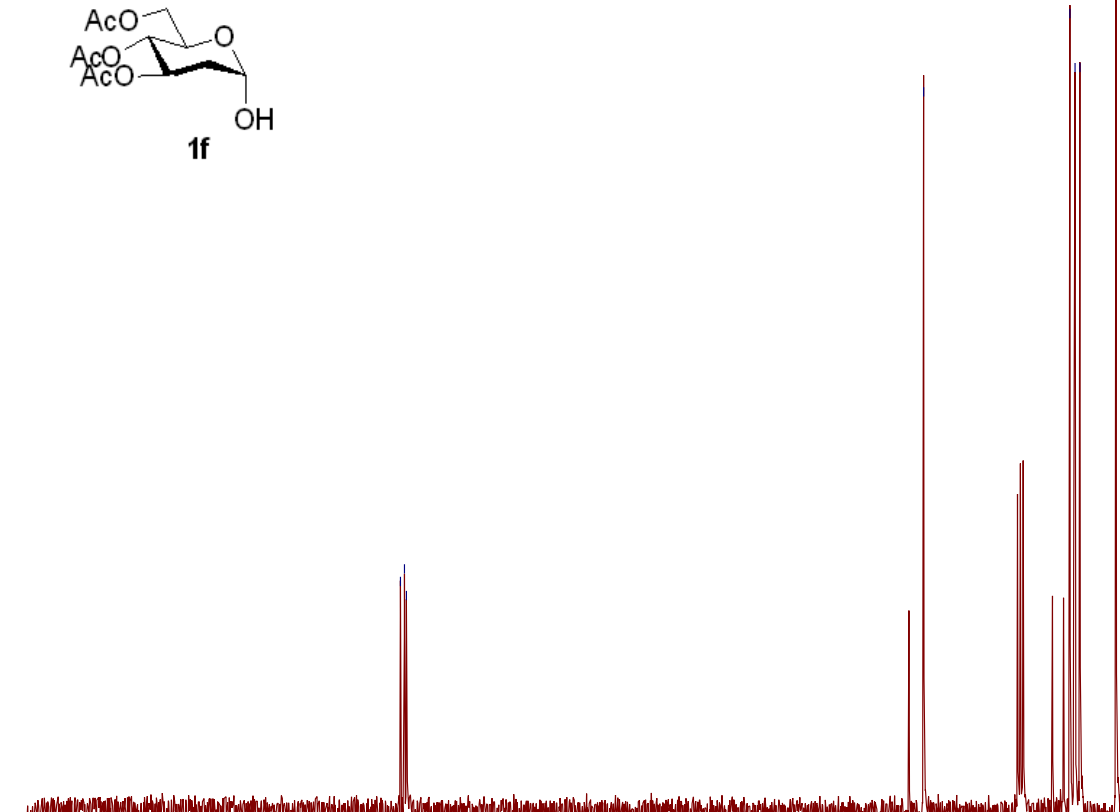

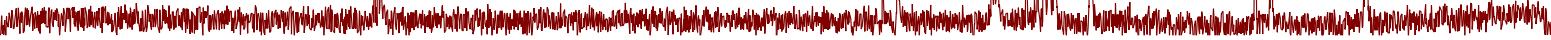

$\begin{array}{lllllllllllllllllllllll}220 & 210 & 200 & 190 & 180 & 170 & 160 & 150 & 140 & 130 & 120 & \begin{array}{c}110 \\ \mathrm{f} 1(\mathrm{ppm})\end{array} & 100 & 90 & 80 & 70 & 60 & 50 & 40 & 30 & 20 & 10 & 0\end{array}$ 2,3,6,2', $3^{\prime}, 4^{\prime}, 6^{\prime}$-Hepta-O-acetylcellobiose.

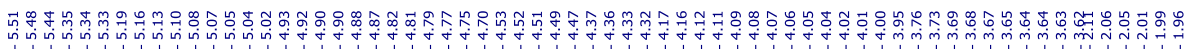

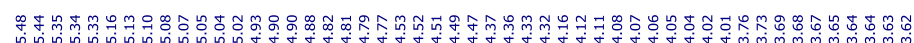

H
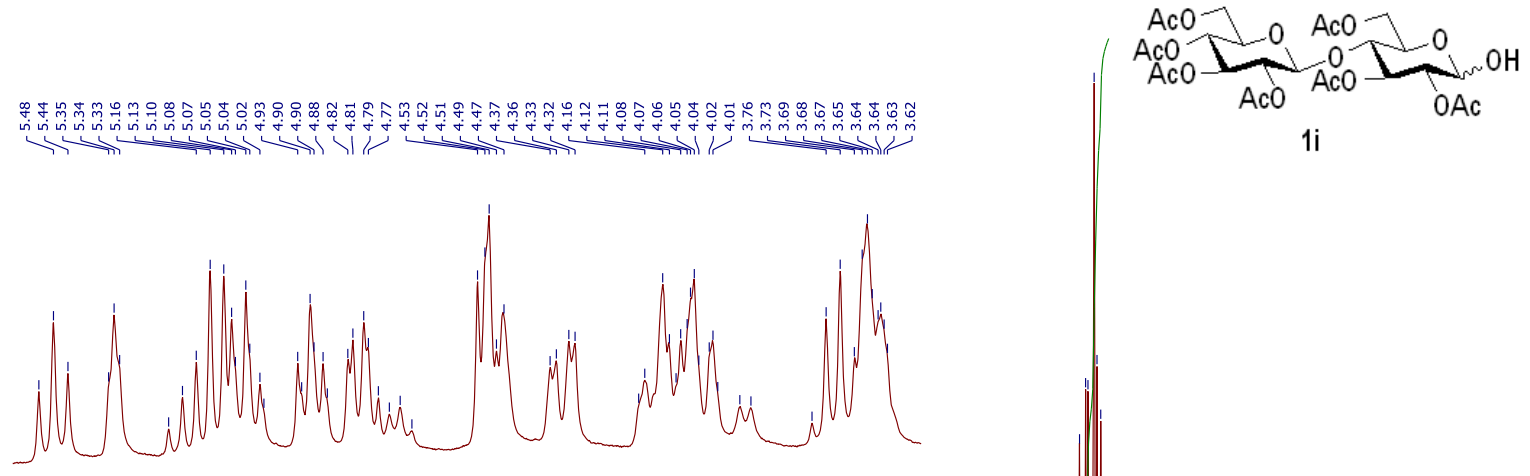

$\begin{array}{lllllllllllllllllllllllll}5.5 & 5.4 & 5.3 & 5.2 & 5.1 & 5.0 & 4.9 & 4.8 & 4.7 & 4.6 & 4.5 & 4.4 & 4.3 & 4.2 & 4.1 & 4.0 & 3.9 & 3.8 & 3.7 & 3.6\end{array}$

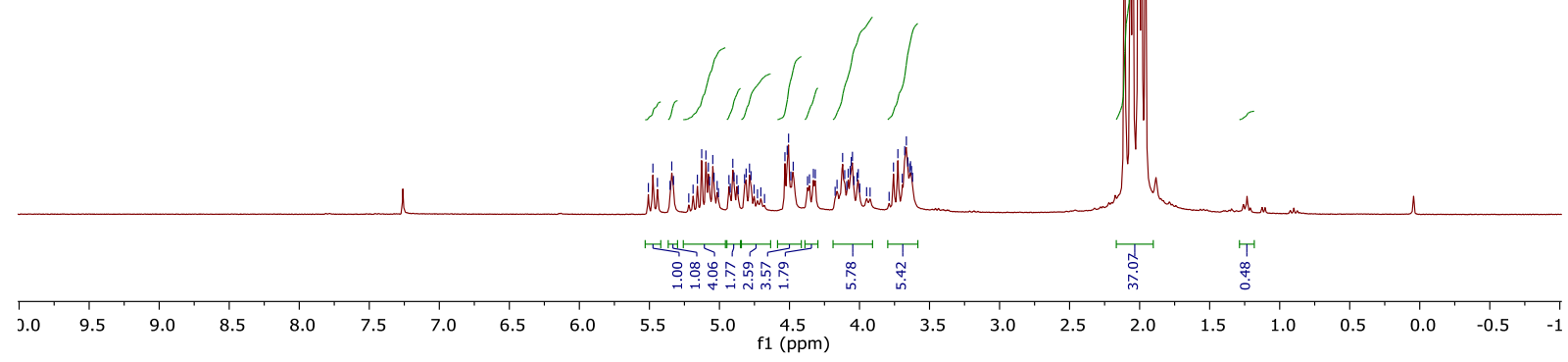




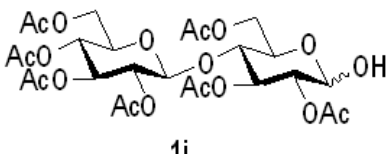

$1 \mathrm{i}$

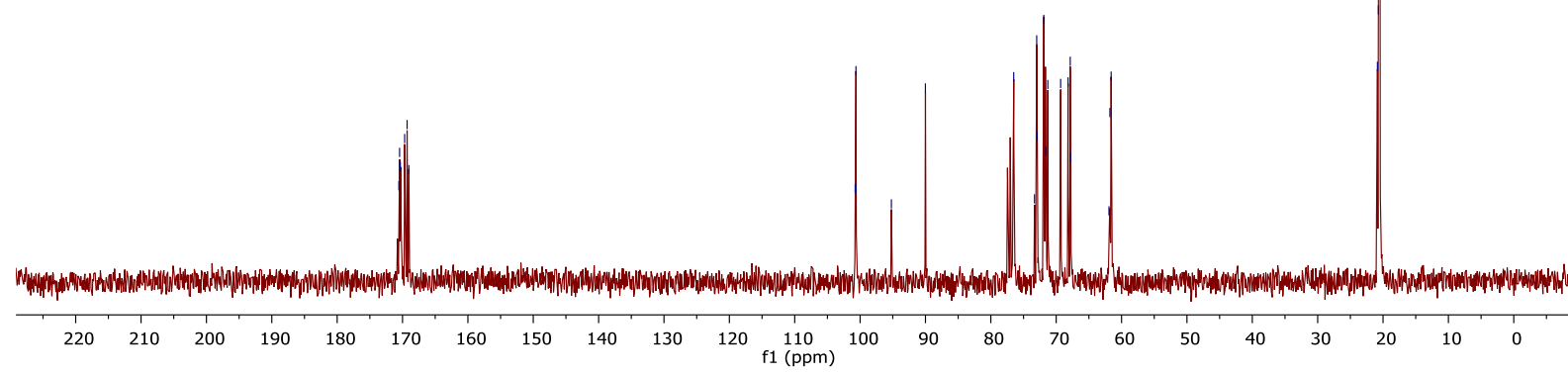

4-O-(4-O-(2,3,4,6-Tetra-O-acetyt-D-glucopyranosyl)-2,3,6-tri-O-acetyla-D-glucopyranosyl)-2,3,6-tri-O-acetyl-D-glucopyranose

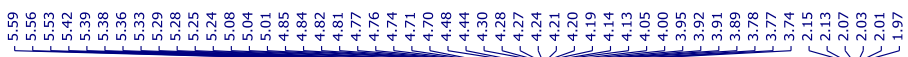
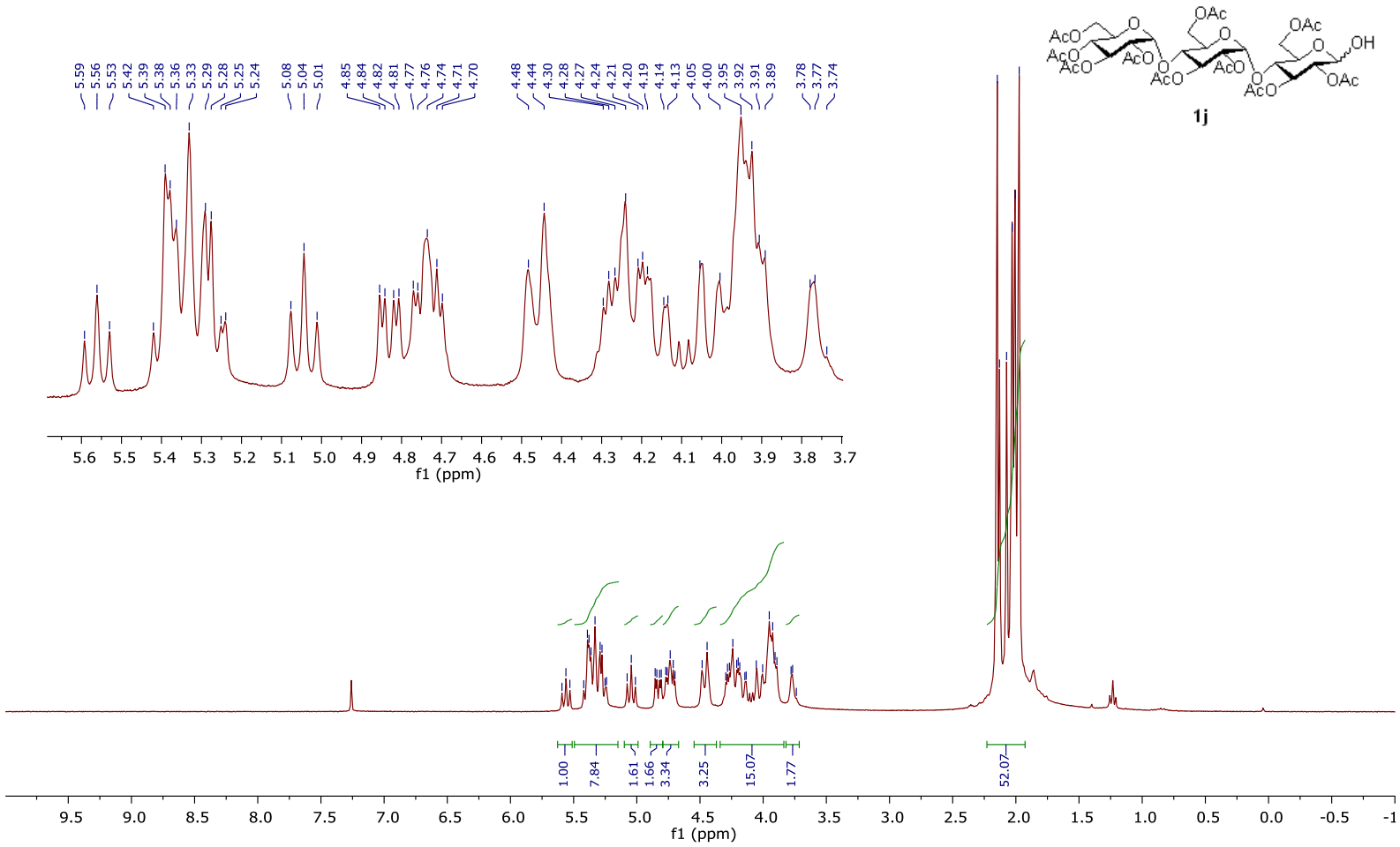
4-O-(4-O-(2,3,4,6-Tetra-O-acetyt-D-glucopyranosyl)-2,3,6-tri-O-acetyla-D-glucopyranosyl)-2,3,6-tri-O-acetyl-D-glucopyranose

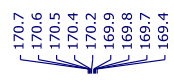

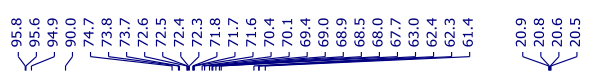
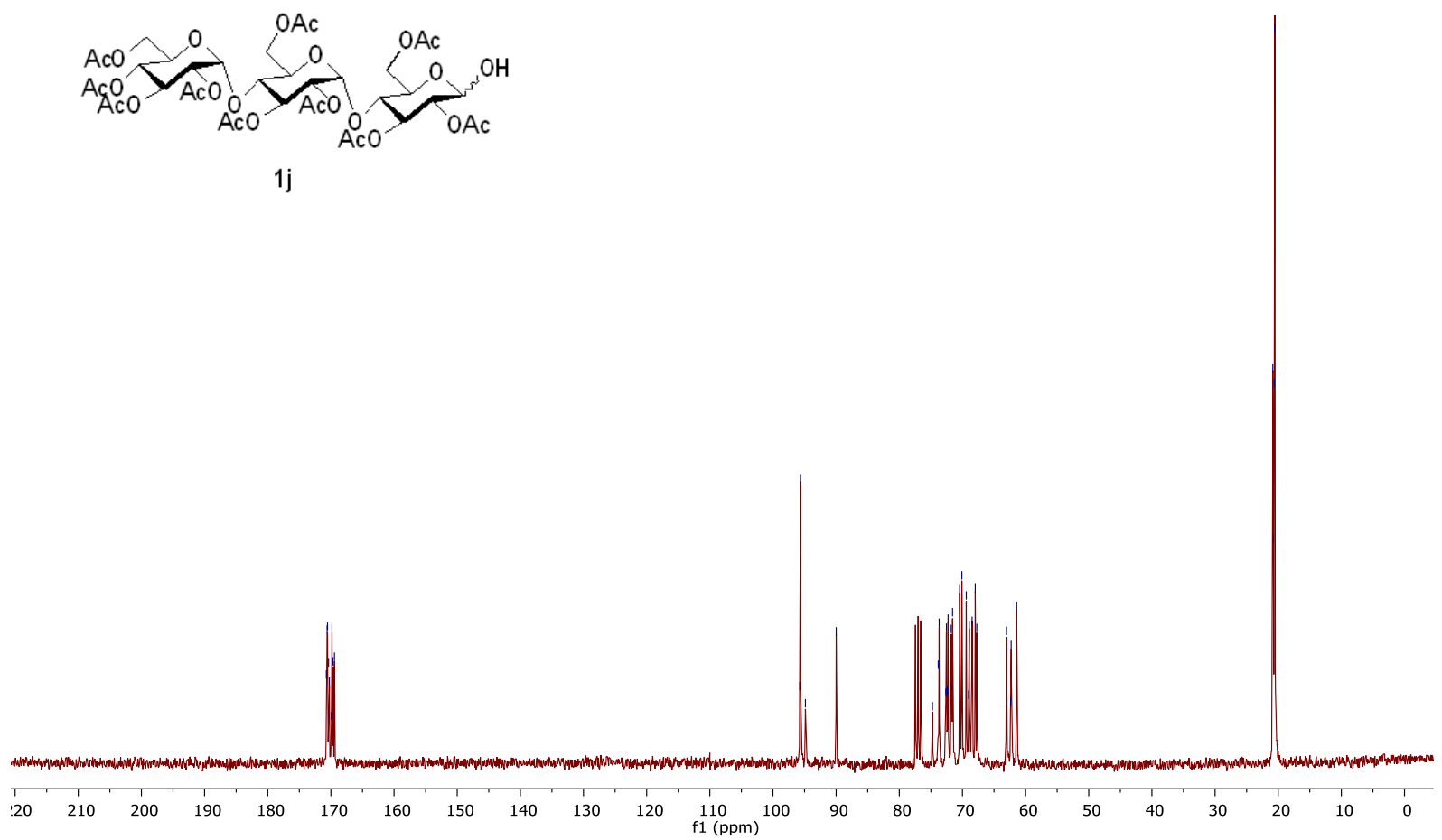

S55 
HPLC chromatograms of compounds $4 f, 4 h$ and $4 i$.
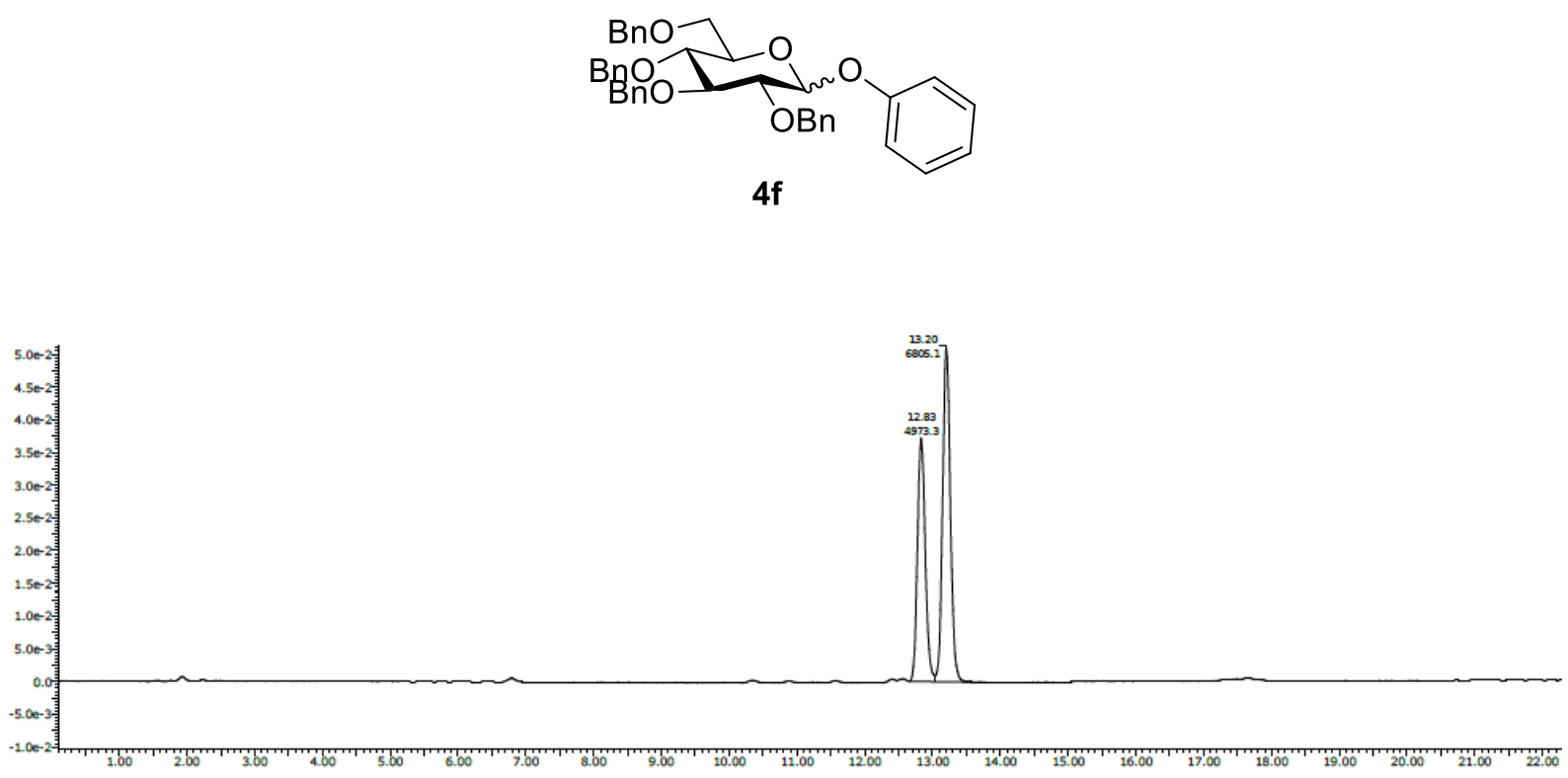

H2O/ACN/0.1\%AF - gr $80-100 \%$ en $10 \mathrm{~min}$ Sunfire C18 - 2.1x150mm - 3.5um - 015334008140

vial 2

3: Diode Array

265

Range: 5.151e-2

Time Height Area Area\%

$\begin{array}{llll}12.83 & 37179 & 4973.34 & 42.22\end{array}$

$\begin{array}{llll}13.20 & 51325 & 6805.13 & 57.78\end{array}$ 

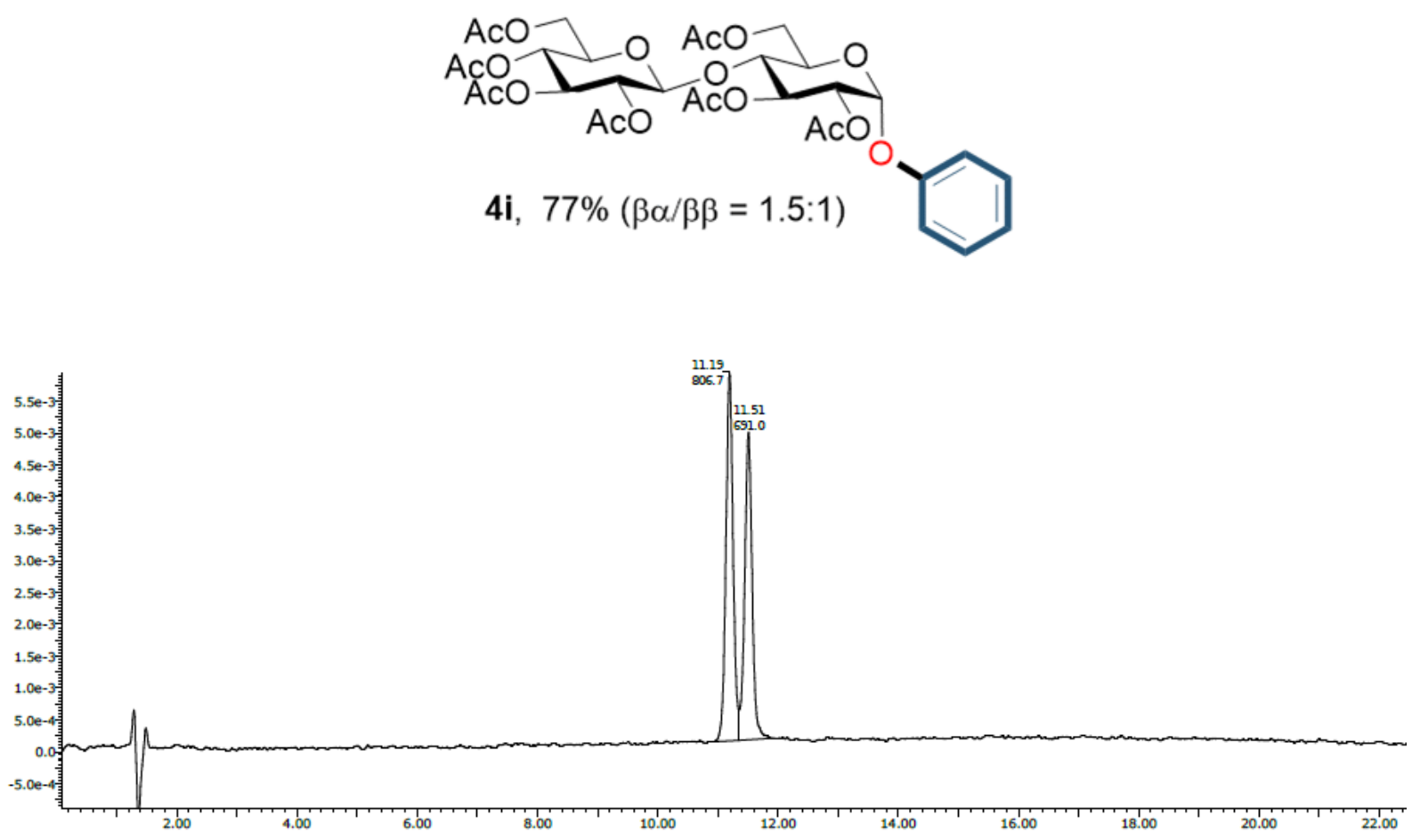

\section{H2O/ACN/0.1\%AF - gr $40-80 \%$ en $20 \mathrm{~min}$ SYNERGI FUSION - 2 X 100mm - 2.5um} vial 3

3: Diode Array 265

Range: $6.947 \mathrm{e}-3$

Time Height Area Area\% $\begin{array}{llll}11.19 & 5781 & 806.74 & 53.86\end{array}$

$\begin{array}{llll}11.51 & 4821 & 690.97 & 46.14\end{array}$ 

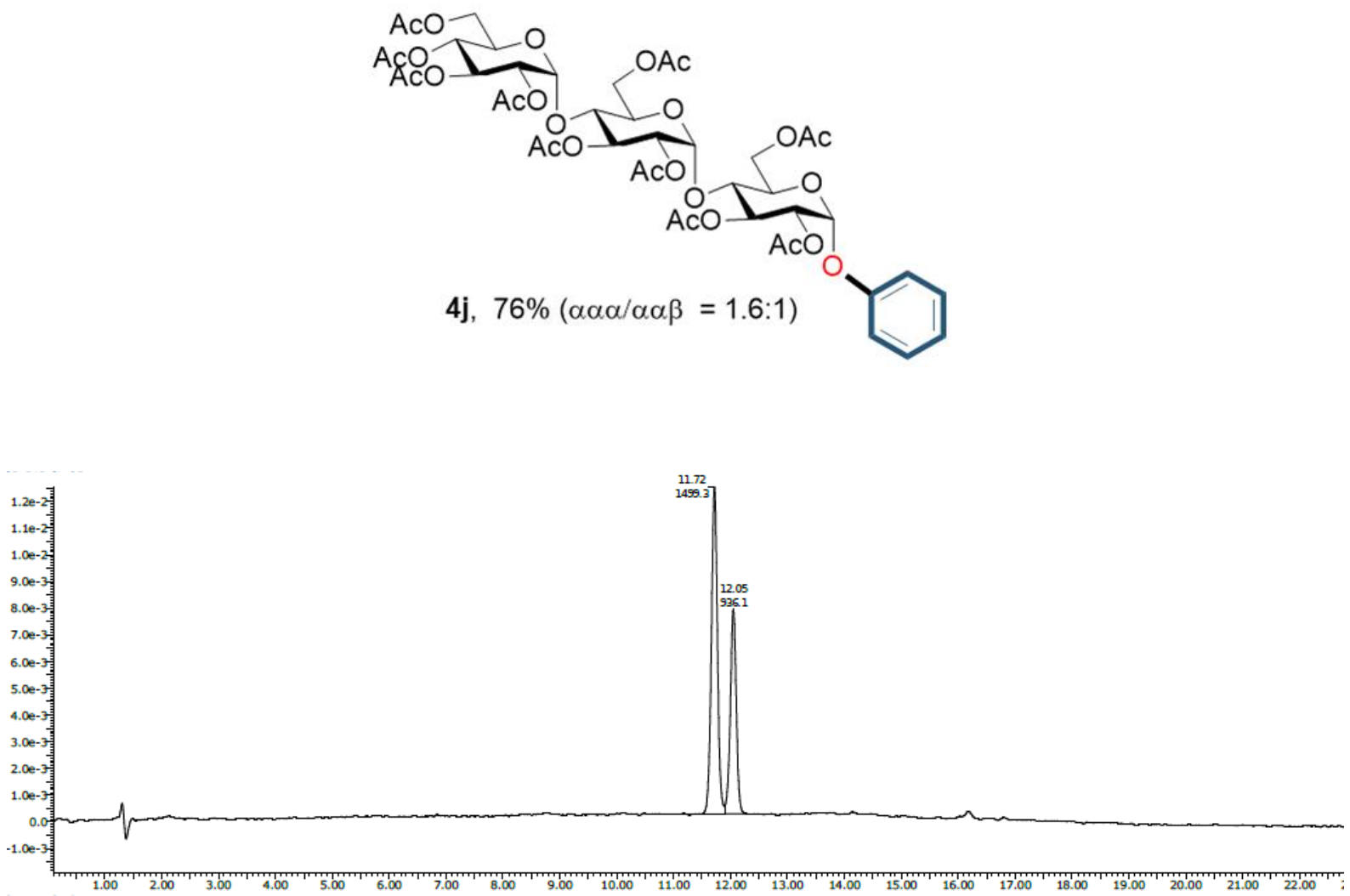

H2O/ACN/0.1\%AF - gr 40-100\% en $20 \mathrm{~min}$ SYNERGI FUSION - 2 X 100mm - 2.5um vial 2

3: Diode Array

265

Range: $1.317 \mathrm{e}-2$

Time Height Area Area\%

$\begin{array}{llll}11.72 & 12236 & 1499.33 & 61.56\end{array}$

$\begin{array}{llll}12.05 & 7674 & 936.12 & 38.44\end{array}$ 
List of starting boronic acids that did not provide the desired coupling product.<smiles>Cc1ccccc1Br</smiles><smiles>COc1cccs1</smiles><smiles>Fc1ccccc1Br</smiles><smiles>Oc1ccc2ncccc2c1</smiles><smiles>Oc1ccncc1</smiles>

$\operatorname{MeB}(\mathrm{OH})_{2}$ 
Study of the stability of starting material $1 \mathrm{a}$ and both anomers of product 3a in $\mathrm{CD}_{2} \mathrm{Cl}_{2}$.

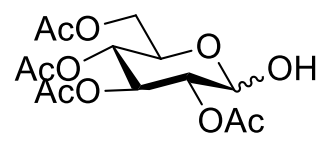

$1 a$

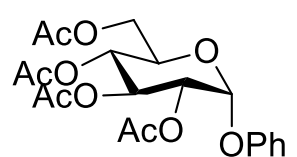

3a-alpha

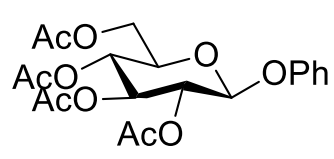

3a-beta

The $\alpha / \beta$ ratio of a three different solutions of compounds $1 \mathbf{a}, 3$ a-alpha and 3 a-beta in deuterated dichloromethane $\left(\mathrm{CD}_{2} \mathrm{Cl}_{2}\right)$ was measured by $1 \mathrm{HNMR}$ after 0 and 19 hours. Results are summarized in the following table.

\begin{tabular}{cccc}
\hline & 1a & 3a-alpha & 3a-beta \\
\hline $\mathbf{t}=\mathbf{0}$ & $\alpha: \beta=1: 3$ & $\alpha: \beta=1: 0$ & $\alpha: \beta=0: 1$ \\
$\mathbf{t}=19 \mathbf{h}$ & $\alpha: \beta=1: 1.1$ & $\alpha: \beta=1: 0$ & $\alpha: \beta=0: 1$ \\
\hline
\end{tabular}

These results could constitute one possible explanation for the epimerization observed during the copper-catalyzed anomeric $O$-arylation of carbohydrate derivatives at room temperature. 
Table for the literature comparison of a selection of synthesized products.

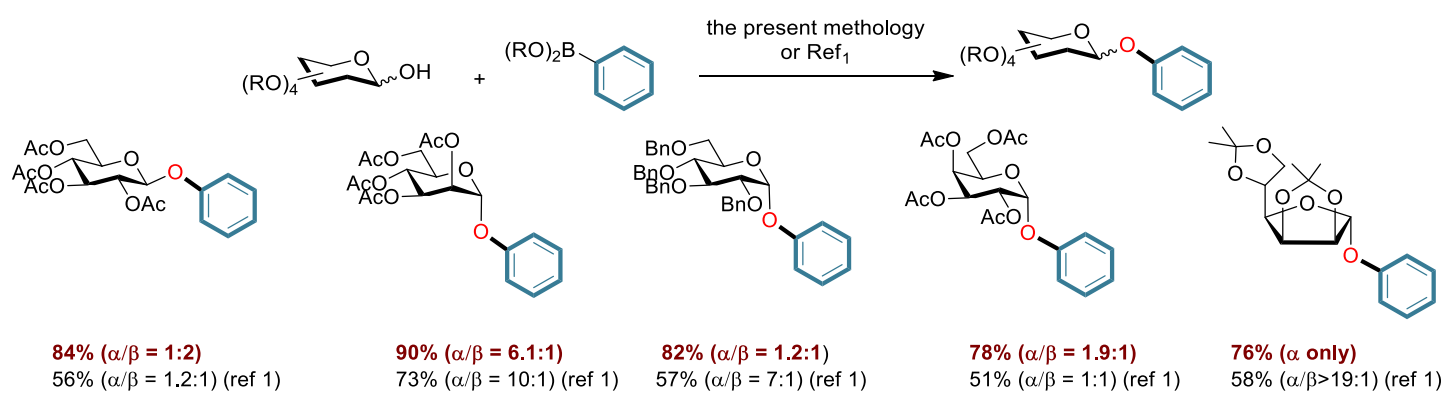

In the red color : the present methodology

Ref 1 : V. Dimakos, J. J. W. Liu, Z. Gea, M. S. Taylor, Copper-mediated anomeric O-arylation with organoboron reagents, Org. Biomol. Chem., 2019, 17, 5671-5674 